\title{
Sensitivity of Space Launch System Buffet Forcing Functions to Buffet Mitigation Options
}

\author{
David J. Piatak $^{1} \quad$ Martin K. Sekula ${ }^{2} \quad$ Russ D. Rausch ${ }^{3}$ \\ Aeroelasticity Branch / NASA Langley Research Center \\ Hampton, VA 23681
}

\begin{abstract}
Time-varying buffet forcing functions arise from unsteady aerodynamic pressures and are one of many load environments, which contribute to the overall loading condition of a launch vehicle during ascent through the atmosphere. The buffet environment is typically highest at transonic conditions and can excite the vehicle dynamic modes of vibration. The vehicle response to these buffet forcing functions may cause high structural bending moments and vibratory environments, which can exceed the capabilities of the structure, or of vehicle components such as payloads and avionics. Vehicle configurations, protuberances, payload fairings, and large changes in stage diameter can trigger undesirable buffet environments. The Space Launch System (SLS) multi-body configuration and its structural dynamic characteristics presented challenges to the load cycle design process with respect to buffetinduced loads and responses. An initial wind-tunnel test of a 3-percent scale SLS rigid buffet model was conducted in 2012 and revealed high buffet environments behind the booster forward attachment protuberance, which contributed to reduced vehicle structural margins. Six buffet mitigation options were explored to alleviate the high buffet environments including modified booster nose cones and fences/strakes on the booster and core. These studies led to a second buffet test program that was conducted in 2014 to assess the ability of the buffet mitigation options to reduce buffet environments on the vehicle. This paper will present comparisons of buffet forcing functions from each of the buffet mitigation options tested, with a focus on sectional forcing function rms levels within regions of the vehicle prone to high buffet environments.
\end{abstract}

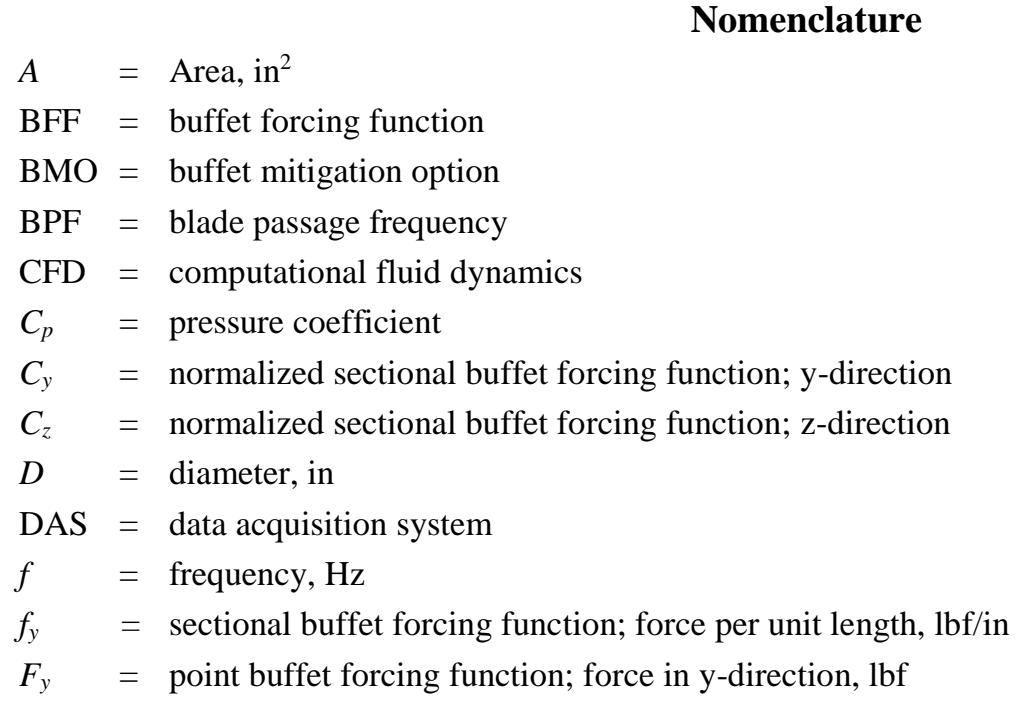

\footnotetext{
${ }^{1}$ Research Aerospace Engineer, Aeroelasticity Branch, david.j.piatak@nasa.gov, Member AIAA

${ }^{2}$ Research Aerospace Engineer, Aeroelasticity Branch, martin.k.sekula@nasa.gov, Senior Member AIAA

${ }^{3}$ Assistant Branch Head, Aeroelasticity Branch, russ.d.rausch@nasa.gov, Associate Fellow AIAA
} 


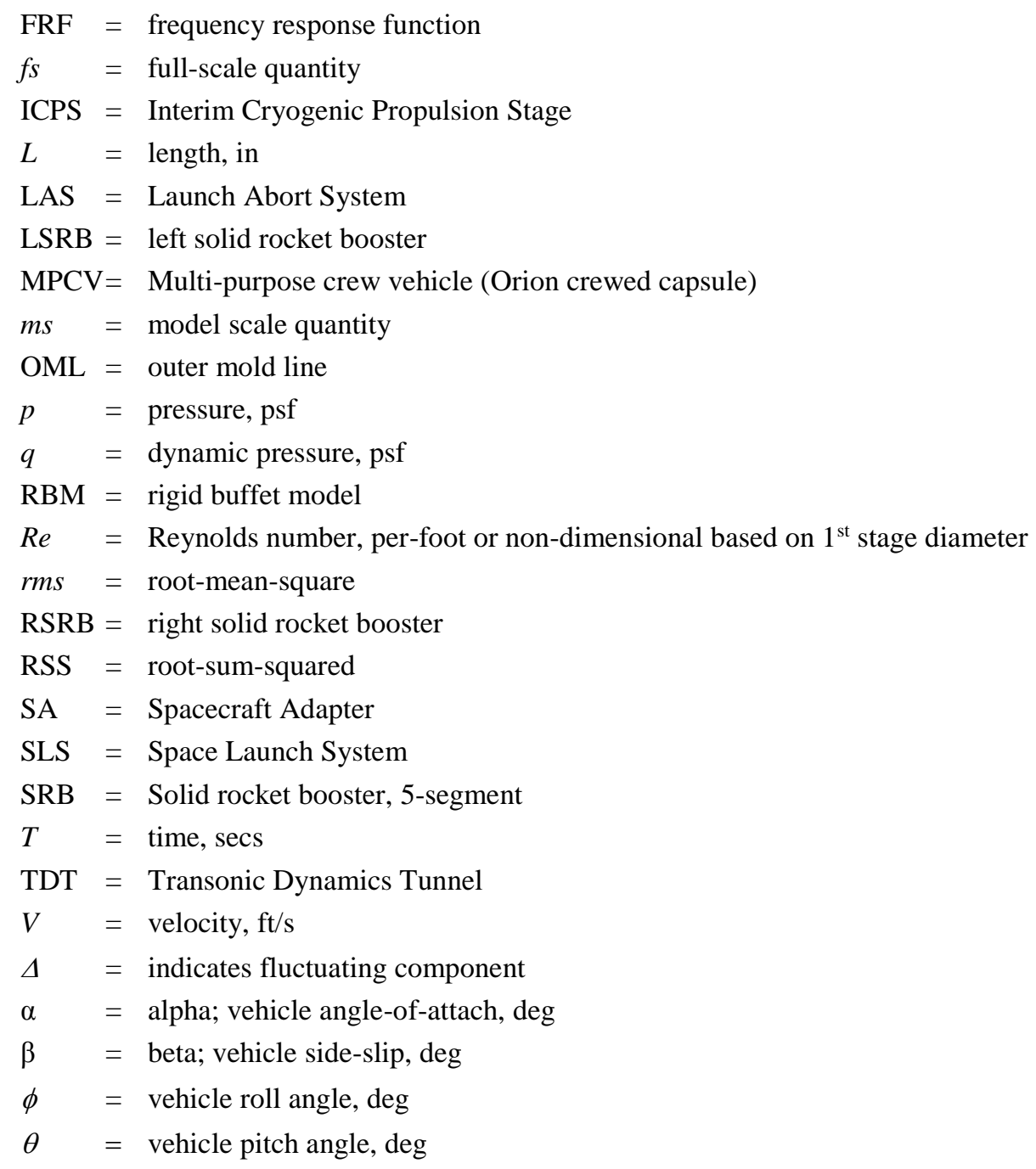

\section{Notice to Readers}

The predicted performance and certain other features and characteristics of the Space Launch System vehicle are defined by the U.S. Government to be Sensitive But Unclassified (SBU). Therefore, values in plots and figures have been removed or normalized to arbitrary values.

\section{Introduction}

$\mathbf{B}$ uffet environments of launch vehicles consist of unsteady aerodynamic phenomenon dominated by fluctuating pressures which arise from high-speed flows over nose cones, interstage geometries, stiffening rings, multi-body boosters, and vehicle protuberances. Fluctuating pressures on and near the vehicle can originate from many sources such as flow separation, flow reattachment, compression/expansion corner geometry, wake effects, turbulence, vortex shedding, and transonic shock oscillations. Even small changes to these geometries can lead to very unique buffet environments which can have an impact on overall vehicle buffet loads and vehicle vibratory response. ${ }^{1-3}$ The vehicle's trajectory conditions such as Mach number, side-slip angle, and angle-of-attack are rapidly changing during its climb to orbit. Therefore, careful attention to these phenomena must be considered at key points in a vehicle's trajectory during the design cycle and vehicle certification process. Transonic buffet loads have been linked to at least two launch vehicle failures: the Mercury/Atlas MA-5 and Atlas/Able vehicles. Both are discussed in Reference 4 by Fleming which provides a concise overview of launch vehicle buffet. Figure 1 illustrates the notional unsteady flow environment of the Space Launch System (SLS) and a simplified structural deflection that may be excited by this buffet environment. 
Buffet forcing functions (BFFs) represent the time-varying forces at the vehicle centerline due to unsteady pressures on the surface of the launch vehicle. These BFFs are one of many load environments that are applied to a structural finite element model of the vehicle as part of the coupled loads analysis (CLA) to determine the structural response and vehicle structural safety margins. ${ }^{5}$ Wind-tunnel testing is the accepted method of determining the BFFs of a launch vehicle configuration by utilizing a highly instrumented rigid model with hundreds of miniature unsteady pressure transducers. ${ }^{6-9}$ Efforts to develop BFFs for the SLS configuration have been underway since 2011 and there have been two primary test programs conducted to obtain unsteady buffet pressures. ${ }^{10-11}$ Buffet tests in 2012 identified high fluctuating pressure environments on the core and booster downstream of the booster forward attachment near Mach 0.92. Coupled loads analysis revealed that when preliminary BFFs developed from these environments were applied to the vehicle structure, buffet loads were largely the source of exceedances of structural and vibratory limits on the SLS vehicle.

Multi-body configurations like the SLS pose a challenging buffet configuration due to the interaction of flow phenomenon in the vicinity of the solid rocket booster (SRB) and core stage interface regions. Flow around the SRB nose cones can be quite asymmetric due to the presence of the core stage and also because the shock that occurs at the expansion corner of the cone-cylinder junction can be marginally stable. ${ }^{12,13}$ This marginally stable or bi-stable shock is easily perturbed by the unsteady environment (vortex-shedding wake) behind the booster forward attachment protuberance. Interactions of SRB nose cone shocks with the core tank and booster forward attachment protuberance environment have been seen to result in high peak $\Delta C_{p, r m s}$ levels on the core and booster downstream of the booster forward attachment protuberance. Strouhal shedding signatures associated with this protuberance have also been noted in the flow field between the SRB and core. These high fluctuating pressure environments observed during the initial SLS buffet test in 2012 triggered an effort to explore mitigation options to reduce the buffet environment associated with the SRB forward attachment and SRB/core shock interactions.

A buffet loads task team was tasked with determining options to reduce these buffet loads via additional analysis during BFF development and/or possible changes to the vehicle outer mold line (OML). Computational fluid dynamic analyses using the code FUN3D proved essential in understanding the flow field in the vicinity of the booster forward attachment and was used to guide the design of booster/core fences and modified booster nose cones as buffet mitigation options (BMOs). ${ }^{14,15}$ Three fence and three booster nose cone configurations were down-selected and tested during the second SLS buffet test program in 2014. These test programs and their preliminary results have been documented in references 10 and 11 .

The intent of this paper is to provide insight into launch vehicle design options which may alleviate high BFF environments associated with multibody booster/core configurations. Specifically, six buffet mitigation option design strategies are presented and compared, each intended to reduce the unsteady environment associated with the booster nose cone expansion shock and the perturbations caused by the unsteady wake of the booster forward attachment. Results presented previously in Reference 10 focused on comparisons of localized sensor fluctuating pressure coefficients $\left(\Delta C_{p, r m s}\right)$ for each of the six BMOs, whereas this paper will present comparisons of BFFs integrated from those pressures to yield a more global interpretation of the BMO effectiveness. Testing was conducted at the NASA Langley Research Center Transonic Dynamics Tunnel (TDT) on a 3-percent scale rigid buffet model of the SLS10005 configuration for the purpose of obtaining unsteady pressure measurements to support buffet environments development for SLS loads cycle analyses. The SLS-10005 configuration served as the baseline for evaluating the effectiveness of the three fence BMO configurations and the three booster nose cone BMO configurations. Timecorrelated unsteady pressure measurements were acquired for each model configuration at 472 locations with adequate spatial and frequency resolution to assess the buffet environment including longitudinal and azimuthal correlation. Pressure measurements have been integrated to yield sectional BFFs at 35 longitudinal stations on the core and boosters. Normalized sectional buffet forcing function root-mean-square trends will be presented for all configurations at Mach numbers between 0.9 and 1.2, vehicle pitch angles of $\theta=0^{\circ}$ and $4^{\circ}$, and vehicle roll angles of $\phi=00^{\circ}, 45^{\circ}$, and $90^{\circ}$.

\section{Test Program Summary}

Primary test objectives of the SLS buffet test program were to acquire time-correlated unsteady pressure measurements on a rigid wind-tunnel model at transonic conditions with sufficient spatial and temporal resolution to enable the development of buffet forcing functions for vehicle loads cycle analyses. A secondary test objective was to acquire a subset of the time-correlated unsteady pressures at aeroacoustic bandwidths for verification of SLS acoustic/vibro-acoustic environments. Buffet loads and vibro-acoustic structural analysis requirements dictate that time-series pressure data must be provided at full-scale rates of $60 \mathrm{~Hz}$ and $2,000 \mathrm{~Hz}$ for buffet and acoustic 
environments, respectively. To achieve these goals, a 3-percent scale model of the SLS-10005 configuration was tested at the Transonic Dynamics Tunnel (TDT) in the Fall of 2014. The model was instrumented with 472 unsteady pressure sensors. Figure 2 shows the SLS rigid buffet model installed in the TDT test section and Figure 3 illustrates the spatial distribution of pressure sensors for buffet and aeroacoustic unsteady pressure measurements. Aeroacoustic sensors doubled as buffet sensors for buffet forcing function development. Data acquisition rates were dictated by the full-scale bandwidth requirements stated above and by the scaling laws listed in Table 1. While holding tunnel test section and model orientation constant, buffet sensor data were acquired at a rate of $16 \mathrm{kHz}$ for twenty seconds and aeroacoustic sensors were acquired at $200 \mathrm{kHz}$ for ten seconds. References 10 and 11 provide thorough summary of the SLS buffet test program from the standpoint of test development, test implementation, and preliminary results.

Six design options were chosen for testing on the SLS-10005 configuration for the purpose of exploring ways to reduce the buffet environments in the wake of the booster forward attachment. The vehicle locations highlighted by station numbers in Figure 3 are those on the core and booster where high buffet environments affect SLS buffet and coupled loads analyses. The baseline SLS-10005 configuration is shown installed in the TDT test section in Figure 2 and a close-up of the booster forward attachment protuberance between the booster and core is shown in Figure 4. Three fence configurations were tested to determine their effectiveness at reducing buffet environments downstream of the forward attachment. Figures 5 and 6 show the sharp and blunt booster fences mounted to the booster with the baseline, shuttle-era nose cones. Both booster fence configurations consisted of a pair of fences mounted to the left and right SRBs (LSRB and RSRB, respectively) beginning at the booster cone-cylinder junction, on either side of the booster forward attachment protuberance. Figure 7 shows the core fences attached to the core stage on both sides of the booster forward attachment protuberance. The fences are intended to contain/limit the unsteadiness of the wake behind the booster forward attachment and result in less perturbation of the cone-cylinder expansion shock and thus lower buffet environments.

Figures 8 through 11 show the four booster nose cone shapes tested on the SLS-10005 configuration including the baseline shuttle-era nose cone shown in Figure 8. All three alternate nose cone shapes were designed to avoid the cone-cylinder junction expansion shock and place any resulting shock further upstream of the booster forward attachment protuberance. Based on unsteady CFD design analyses (Reference 14), the canted ogive shape shown in Figure 9 was felt to be the most promising of the three, while the bent bi-conic nose cone shape, shown in Figure 10, was a compromise to the canted ogive from the standpoint of ease of manufacture. The third alternate nose cone shape is the canted straight design shown in Figure 11, which was expected to provide the same benefits of the canted ogive, yet also more easily fabricated. Each alternate nose cone had the same number of unsteady sensor ports as the baseline nose cones, but at very different locations. These differences in nose cone geometries and sensor locations prevent the comparison of integrated sectional buffet forcing functions for stations on the alternate nose cones for this paper.

Buffet data were acquired for each configuration described above at the NASA Langley TDT, which has been utilized for many past launch vehicle buffet test programs including Saturn/Apollo, Atlas Centaur, Ares I, Dream Chaser Atlas V, and now Space Launch System. The TDT is a closed-circuit, continuous flow transonic wind-tunnel designed to address the aeroelastic and dynamic load test needs of rotorcraft, aircraft, and launch vehicles. ${ }^{17-19}$ Test section flow conditions at several SLS test matrix Mach numbers are provided in Table 2. All test data were acquired in an R134a test medium at conditions near the extremes of the TDT performance boundary to achieve maximum possible Reynolds numbers. Results presented in this paper will focus on Mach numbers of 0.90, 0.95, 1.10, and 1.19 and vehicle pitch/roll combinations of $0^{\circ} / 0^{\circ}, 4^{\circ} / 0^{\circ}, 4 / 45^{\circ}$, and $4 \% 90^{\circ}$.

\section{Buffet Forcing Function Development}

Buffet forcing functions (BFFs) consist of orthogonal force time histories acting at the centerline of a launch vehicle as shown in the notional vehicle segment in Figure 12. The BFF time histories represent the unsteady aerodynamic loads acting on each segment of the vehicle due to the buffet environment. There are four primary tasks associated with BFF development: (1) integration of pressures to obtain centerline BFFs, (2) applying buffet scaling laws to scale the model-scale BFFs to full-scale using the vehicle trajectory conditions, (3) filtering out facility drive system tones from the BFF time histories, and (4) applying coherence factors based on sensor-to-sensor spatial coherence to reduce over-conservatism in BFFs. For completeness, BFF coherence factors are discussed in this section. However, since coherence factors are only relevant to point force BFFs, they are not applied to the sectional BFF trends discussed later in this paper. 


\section{A. Pressure Integration}

Buffet forcing functions are obtained by integrating measured pressures from rings of transducers distributed along the longitudinal axis of the model. For the SLS buffet model instrumentation locations shown in Figure 3, there are 35 unique longitudinal stations which possess rings of 4,8 , and even 16 unsteady pressure sensor ports. The buffet forcing functions can be calculated using two methods of pressure integration yielding either sectional (force/length) or point (force) loads; each useful for different types of analyses and comparisons. A sectional load $\left(f_{x}, f_{y}\right.$, and $\left.f_{z}\right)$, depicted in Figure 13a, is calculated by integrating the measured pressures along the circumference at a station to produce a set of orthogonal loads acting at the centerline of the segment. Sectional buffet forcing functions have units of force-per-length and are useful for comparing the relative magnitude of the buffet loads at various vehicle stations, independent of sensor distribution. Point force BFFs $\left(F_{x}, F_{y}\right.$, and $\left.F_{z}\right)$, shown in Figure 13b, are calculated by integrating the measured pressures circumferentially and longitudinally over a segment of the model. These orthogonal point BFFs are used to conduct the buffet loads analysis for input to vehicle coupled loads analyses. Because unsteady pressure sensor stations are not distributed equally longitudinally, point force BFF magnitudes are greatly affected by this fact and are not useful for station-to-station comparisons of BFF trends. Thus, only sectional BFFs are presented in this paper to compare the effectiveness of BMOs at reducing the BFF environments for the SLS vehicle.

\section{B. Buffet Forcing Function Scaling Laws}

Model-scale wind-tunnel test data must be scaled to full-scale conditions to be used by structural loads analyses. Buffet scaling laws are provided in Table 1, which relates model-scale parameters to full-scale parameters. Full-scale launch vehicle trajectory conditions (Mach, $\mathrm{q}, \mathrm{V}$, etc. versus time) are determined by interpolation using the modelscale Mach number. Scale factors for load, pressure, time, and frequency are then computed using the interpolated full-scale conditions along with the known model-scale flow conditions from the wind-tunnel. In the case of time dependent data such as BFFs, both the magnitude and sample period (scan rate) are scaled using the method described above.

\section{Removal of Wind Tunnel Drive Tones}

Besides pressure integration and scaling of the BFFs from model-scale to full-scale, several other aspects of BFF development must be considered prior to the release of the BFF database for vehicle load cycle analyses. First, there are wind-tunnel facility drive system tones that are present in any transonic wind tunnel that must be removed from the pressure time histories prior to BFF integration. During buffet loads analyses, vehicle structural modes of vibration present in the finite element model may be excited by these facility tones, leading to load and response environments not representative of actual flight conditions. The tones that are most easily filtered from the pressure time histories are blade passage frequencies (BPFs) which arise due to the passage of the facility drive fan blades in vicinity of fixed stator vanes. These tones (and their harmonics) are easily identified and are a function of drive motor speed and the number of blades on the compressor fan. Reference 7 provides a description of the process used to filter BPF signatures from SLS test data.

\section{Reducing Over-Conservatism in Buffet Forcing Functions}

A second important consideration in the development of buffet forcing functions is the reduction of the overconservatism that is present if the individual unsteady pressures are integrated across its entire zone of integration. This over-conservatism arises because the integration process assumes that, at any instant in time, the measured pressure is applied to the entire area of integration. However, the actual magnitude and phase distribution of the unsteady pressure on that integration region is not adequately represented by the single discrete measurement at the sensor orifice. Thus, the assumption to integrate the single pressure measurement time history across the entire zone of integration would lead to an over-prediction of the buffet forces on that panel/region. If this over-conservatism is not dealt with appropriately, buffet environments may erroneously result in high buffet loads which reduce vehicle structural margins and increase the vehicle structural weight. Sensor-to-sensor coherence-based techniques have been developed at NASA Langley to reduce the integration area of each sensor as part of the development of buffet forcing functions for launch vehicles. The methods used for computing longitudinal spatial coherence factors is documented in Reference 8. 


\section{Comparisons of Buffet Forcing Function Results}

Buffet environments of the baseline SLS-10005 configuration and six BMO configurations are compared in this section and focus on the root-mean-square (rms) values of the fluctuating component of sectional buffet forcing functions at vehicle longitudinal stations. Sectional BFFs are chosen because they do not include the effect of sensor distribution on BFF magnitude unlike point load BFFs. Because of differences in sensor locations for each of the alternate nose cones, sectional BFF results are not shown at these stations for the alternate nose cones. Prior to calculation of the rms values of sectional BFFs, the time histories are scaled to full-scale and are band-pass filtered to $0.5-60 \mathrm{~Hz}$ and the resulting full-scale sectional BFFs have been normalized to non-dimensional units prior to calculation of the rms values. Results are presented in the form of lateral (y-direction) and vertical (z-direction) rms trends in normalized sectional BFFs $\left(\Delta C_{y, r m s}\right.$ and $\left.\Delta C_{z, r m s}\right)$ for the RSRB, core, and LSRB. The vehicle reference coordinate system is shown in Figure 3. Results in the x-direction are not shown for brevity due to the fact that these loads only occur on conical or ogive sections of the vehicle, tend to be reduced in magnitude compared to out-of-plane BFFs, and do not significantly contribute to excitation of vehicle bending modes and vibrations.

\section{A. Buffet Forcing Function Trends with Mach Number for the Baseline SLS-10005 Configuration}

Normalized sectional buffet forcing function rms trends are shown in Figures 14 through 21 for the baseline SLS10005 configuration with standard shuttle-era nose cones for Mach numbers of 0.90, 0.95, 1.10, and 1.19 at four combinations of pitch and roll. The equivalent vehicle angle-of-attack and sideslip (alpha and beta) are provided in Table 3. Figures 14 and 15 show BFF trends for vehicle pitch and roll of $\theta=0^{\circ}$ and $\phi=0^{\circ}$. A very defined peak in $\Delta C_{y, r m s}$ is shown at Mach 0.95 on the core and boosters just downstream of the booster forward attachment. Buffet forcing function fluctuating magnitudes in the y-direction $\left(\Delta C_{y, r m s}\right)$ exhibit high peak values because the interaction of the booster nose cone expansion shock with the unsteady wake of the booster forward attachment protuberance is not symmetric between the right and left booster. When these unsteady pressure environments are integrated to yield buffet forcing functions, the result is a lateral, y-direction, force resultant with relatively high magnitude and unsteady signature. This environment was found to result in high buffet loads and vehicle vibratory responses and triggered the SLS program to pursue options for reducing this environment to maintain adequate vehicle structural safety margins. The peak in $\Delta C_{y, r m s}$ and $\Delta C_{z, \text { rms }}$ at Mach 0.90 on the Orion service module is due to the presence of a terminal shock at that measurement port which causes local unsteadiness in the flow. As expected for the $\theta=0^{\circ}$ and $\phi=0^{\circ}$ vehicle orientation, the BFF rms trends were reasonably symmetric on the RSRB and LSRB.

For vehicle orientation of $\theta=4^{\circ}$ and $\phi=0^{\circ}$, Figures 16 and 17 show symmetric trends in sectional BFFs rms magnitudes with peaks at the anticipated regions downstream of the booster forward attachment. As vehicle roll is increased to $\phi=45^{\circ}$ while holding pitch at $\theta=4^{\circ}$, the RSRB is in a windward position with respect to the flow and the LSRB is in leeward position and is somewhat in the wake of the booster. This results in slightly higher unsteady BFF magnitudes on the RSRB than on the LSRB as shown in Figures 18 and 19. At a pitch and roll angle of $\theta=4^{\circ}$ and $\phi=90^{\circ}$, the vehicle is at a side-slip angle $\beta=4^{\circ}$ with the RSRB in windward position and LSRB leeward and in the partial wake of the core. At this vehicle orientation, the $\Delta C_{y, \text { rms }}$ magnitudes on the RSRB show a very distinct peak downstream of the booster forward attachment that is significantly higher in magnitude than that on the LSRB. These results clearly show that the booster unsteady BFF magnitudes are very sensitive to vehicle orientation, in particular vehicle side$\operatorname{slip}(\beta)$.

\section{B. Comparison of Fence and Booster Nose Cone Buffet Forcing Function Trends to Baseline Configuration}

Comparisons of the effectiveness of the six buffet mitigation options (BMOs) are presented in this section with focus on the buffet environment on the core and boosters downstream of the booster forward attachment. The key objective of this BMO study was to provide the SLS program with methods of reducing the buffet environment associated with the booster forward attachment. Results are presented as normalized sectional buffet forcing function rms trends. Buffet mitigation options include three booster/core fence configurations including the sharp booster fences, blunt booster fences, and the core fences. Each fence configuration consists of a pair of fences on the left and right booster, which are mounted on either side of the booster forward attachment protuberance. Figures 5 through 7 show the three fence configurations as tested on the SLS-10005 configuration. In addition to the fence configurations, three alternate booster nose cones were considered including the canted ogive, bent bi-conic, and canted straight configurations shown in Figures 9 through 11. Because sensor locations on the booster nose cones differed between each configuration due to their unique shapes, no attempt has been made to integrate these pressures to yield buffet forcing functions for comparison. Stations of most interest for reducing buffet environments are shown in Figure 3 and primarily include stations $24,74,25,75$, and 26. Data are presented in form of $\Delta C_{y, r m s}$ and $\Delta C_{z, r m s}$ trends on 
RSRB, core, and LSRB with a focus only on the region of vehicle downstream of booster forward attachment affected by the BMOs.

Results of sectional normalized BFF rms trends for Mach 0.90 are presented in Figures 22 through 27 for $\theta / \phi$ values of $0 / 0^{\circ}, 4^{\circ} / 0^{\circ}$, and $4 \% 90^{\circ}$, which provides examples of flight attitudes at zero angle with respect to flow, at $4^{\circ}$ angle-ofattack, and at $4^{\circ}$ sideslip. All BMOs are shown to reduce the buffet environments downstream of the booster forward attachment. However, some configurations results in higher values of $\Delta C_{y, r m s}$ at the station 23, just forward of the booster attachment. The canted straight nose cone is shown to increase buffet forcing function environments on the boosters, while not being as effective as other BMOs on the core.

Mach 0.95 BFF results are shown in Figures 28 through 33. At this Mach number, the baseline configuration's buffet environment produces high buffet loads which result in reduced vehicle structural margins. High BFF fluctuating trends in the y-direction, as shown in Figure 28 and in pressure coefficient results $\left(\Delta C_{p, r m s}\right)$ in Ref. 10, are most severe from the standpoint of fluctuating magnitudes and with respect to frequency content which drives SLS vehicle structural modes. The fences and nose cones are shown to be effective at reducing the buffet environments at this critical transonic flight regime. Results at the $4^{\circ}$ sideslip vehicle attitude $\left(\theta / \phi=4^{\circ} / 90^{\circ}\right)$, show considerable asymmetry between the windward RSRB side and the leeward LSRB, which is somewhat in the wake of the vehicle core. Additionally, BMO configurations are shown to increase the BFF rms trends at station 23 on the LSRB.

Sectional buffet forcing functions at supersonic conditions are not as severe or critical to structural margins as those at transonic conditions, but reductions in these environments have been demonstrated. Figures 34 through 39 present BMO sectional BFF trends $\left(\Delta C_{y, r m s}\right.$ and $\left.\Delta C_{z, r m s}\right)$ at a Mach number of 1.10 and for $\theta / \phi$ values of $0 \% 0^{\circ}, 4^{\circ} / 0^{\circ}$, and 4\%90. Figures 40 through 45 show trends in BFFs at Mach 1.19.

\section{Summary of Buffet Mitigation Option Sectional BFF Trends}

In order to more clearly show the impact of the fence and alternate nose cone configurations on buffet environments in the wake of the booster forward attachment, a percent reduction in root-sum-squared (RSS) values has been chosen as a metric. The RSS magnitudes of $\Delta C_{y, r m s}$ and $\Delta C_{z, r m s}$ at each BMO configuration and flight condition have been computed and then compared to the baseline SLS-10005 value to arrive at a percent reduction to be representative of the relative effectiveness of each buffet mitigation option considered in this test program. These results are summarized in Figures 46 through 48 for the buffet forcing function RSS trends for the core, RSRB, and LSRB.

At the Mach 0.90 and 0.95 transonic conditions, the canted straight nose cones exhibit the least percent reduction of all BMOs tested. This is suspected to be a result of the shock between the canted straight nose cone and core surface remaining perturbed by the unsteady wake of the booster forward attachment. Since a high priority is placed on the transonic buffet environments, the canted straight nose cone is clearly not a recommended option to reduce buffet loads on the SLS vehicle. The booster fences, core fences, and canted ogive nose cones are shown to be effective at reducing buffet environments in the wake of the booster forward attachments from between 30 to 60 percent on the core, RSRB, and LSRB. The bent bi-conic nose cone results in less reduction in the buffet environment within the transonic regime, but is a viable option if other design concerns are considered. Ultimately, BMO effectiveness, structural weight impacts, and cost must be carefully considered when making changes to the vehicle structure and outer mold line shape.

\section{Conclusions}

A wind-tunnel study of the effectiveness of six design options to reduce Space Launch System buffet environments has been conducted and fluctuating pressures at 472 measurement locations have been integrated to yield buffet forcing functions. Three booster/core fence configurations and three alternate booster nose cone shapes were designed and tested during rigid buffet model testing at the NASA Langley Research Center's Transonic Dynamics Tunnel. The analysis of the results of this data indicates:

Sectional buffet forcing functions reveal areas on the vehicle of peak buffet environments in the wake of the booster forward attachment points, particularly at transonic conditions. This buffet environment results in low structural margins based on initial buffet and coupled loads analyses, which prompted the program to consider methods of reducing these buffet loads.

At some test conditions (particularly non-zero angle-of-attack and sideslip), fences and alternate nose cones have been shown to elevate the buffet environments on the booster at the measurement location immediately forward of the booster attachment (station 23). 
The LSRB and RSRB unsteady BFF magnitudes in y-direction $\left(\Delta C_{y, r m s}\right)$ are sensitive to vehicle orientation, in particular vehicle side-slip ( $\beta$ ). At non-zero side-slip angles, the peak in BFF magnitude on left and right SRBs are asymmetric with the wind-ward side having a significantly higher and more pronounced peak.

The sharp and blunt booster fences and the core fence proved effective at reducing the high buffet environments in the wake of the booster forward attachment at transonic conditions.

Of the three nose cones tested, the canted ogive and bent bi-conic resulted in the most consistent reduction in the buffet environment in the transonic regime. The canted straight nose cone configuration yielded significantly lower reductions in the buffet environment in wake of booster forward attachment at transonic conditions.

\section{References}

1. Rainey, Gerald A., "Progress on the Launch-Vehicle Buffeting Problem." Journal of Spacecraft and Rockets, Volume 2, Number 3, May-June 1965.

2. Cole, Henry A., Jr.; Erickson, A. L.; and Rainey, A. G.: "Buffeting During Atmospheric Ascent." NASA SP8001, 1963, Revised November 1970.

3. Jones, George W.; Foughner, Jerome T.; Jr., "Investigation of Buffet Pressures on Models of Large Manned Launch Vehicle Configurations." NASA TN-D-1663, May 1963.

4. Fleming, E.R., "Transonic Buffeting Loads Experience at The Aerospace Corporation." The Aerospace Corporation Report No. TOR-95(5530)-6. March 1995.

5. Kabe, A.M., "Time Domain Buffet Loads Analysis." The Aerospace Corporation Report Number TOR2007(2209)-6078. November 17, 2006.

6. Schutzenhofer, Luke A.; Howard, Paul W.: "Acquisition and Reduction of Large Volumes of Fluctuating Data." Aerophysics Research at MSFC: Research Achievements Review. Volume III, Report No. 4. NASA TM X53799, 1968.

7. Piatak, D.J.; Sekula, M.K.; and Rausch, R.D.: "Ares Launch Vehicle Transonic Buffet Testing and Analysis Techniques." Journal of Spacecraft and Rockets. Vol. 49. No 5. September-October 2012.

8. Sekula, M. K.; et al, "NASA Test Summary Document for the 3.5 Percent Ares I-X Rigid Buffet Model; Transonic Dynamic Test \#599; November-December 2007,” NASA TP-2015-218703, March 2015.

9. Sekula, M.K.; Piatak, D.J.; Rausch, R.D.: "Analysis of Crew Launch Vehicle Transonic Alternating Flow Phenomenon." Journal of Spacecraft and Rockets. Vol. 49. No. 5. September-October 2012.

10. Piatak, D.J.; Sekula, M.K.; Rausch, R.D.; Florance, J.R.; and Ivanco, T.G.: "Overview of the Space Launch System Transonic Buffet Environment Test Program". AIAA 2015-0557. Presented at the $53^{\text {rd }}$ AIAA Aerospace Sciences Meeting, January 5-9, 2015.

11. Sekula, M.K.; Piatak, D.J.; Rausch, R.D.: "Initial Assessment of Space Launch System Transonic Unsteady Pressure Environment". AIAA 2015-0558. Presented at the 53 ${ }^{\text {rd }}$ AIAA Aerospace Sciences Meeting, January 59, 2015.

12. Chevalier, H.L.; and Robertson, J.E.: "Pressure Fluctuations Resulting from an Alternating Flow Separation and Attachment at Transonic Speeds". Arnold Engineering Development Center Technical Documentary Report No. AEDC-TDR-63-204. November 1963.

13. Sekula, M.K.; Piatak, D.J.; and Rausch, R.D.: "Analysis of Ares Crew Launch Vehicle Transonic Alternating Flow Phenomenon". AIAA Journal of Spacecraft and Rockets, Vol. 49, No. 5, September-October 2012.

14. Brauckmann, G.J.; Streett, C.; Kleb, W.L.; Alter, S.J.; Murphy, K.J.; and Glass, C.E.: "Computational and Experimental Unsteady Pressures for Alternate SLS Booster Nose Shapes". AIAA 2015-0559. Presented at the $53^{\text {rd }}$ AIAA Aerospace Sciences Meeting, January 5-9, 2015.

15. Alter, S.J.; Brauckmann, G.J.; Kleb, B.; Glass, C.E.; Streett, C.L.; and Schuster, D.M.: "Time-Accurate Unsteady Pressure Loads Simulated for the Space Launch System at Wind Tunnel Conditions". AIAA 20153149. Presented at the $33^{\text {rd }}$ AIAA Applied Aerodynamics Conference, 22-26 June 2015.

16. Piatak, D.J.; Sekula, M.K.; and Rausch, R.D.: “Comparison of Ares I-X Wind-Tunnel-Derived Buffet Environment with Flight Data." AIAA Journal of Spacecraft and Rockets. Vol. 49. No 5. September-October 2012. 
17. Cole, Stanley R.; Keller, Donald F.; and Piatak, David J.: "Contributions of the NASA Langley Transonic Dynamics Tunnel to Launch Vehicle and Spacecraft Development." AIAA 2000-1772, Presented at the AIAA Dynamics Specialists Conference, Atlanta, GA, April 5-6, 2000.

18. Cole, Stanley R.; Garcia, Jerry L.: "Past, Present, and Future Capabilities of the Transonic Dynamics Tunnel from an Aeroelasticity Perspective." AIAA 2000-1767, Presented at the AIAA Dynamics Specialists Conference, Atlanta, GA, April 506, 2000.

19. Cole, Stanley R.; Johnson, R. Keith; Piatak, David J.; Florance, Jennifer P.; and Rivera, José A., Jr.: "Test Activities in the Transonic Dynamics Tunnel and a Summary of Recent Facility Improvements." AIAA 20031958, Presented at the AIAA Dynamics Specialists Conference, Norfolk, VA, April 9-10, 2003.

Table 1. Rigid buffet model scaling laws.

\begin{tabular}{|c|c|}
\hline $\begin{array}{c}\text { Quantity to } \\
\text { be Scaled }\end{array}$ & Full scale to Model scale Relationship \\
\hline \hline Pressure & $p_{f s}=p_{m s} \frac{q_{f s}}{q_{m s}}$ \\
\hline Force & $F_{f s}=F_{m s} \frac{q_{f s}}{q_{m s}}\left(\frac{D_{f s}}{D_{m s}}\right)^{2}$ \\
\hline Time & $T_{f s}=T_{m s} \frac{D_{f s}}{D_{m s}} \frac{V_{m s}}{V_{f s}}$ \\
\hline Frequency & $f_{f s}=f_{m s} \frac{D_{m s}}{D_{f s}} \frac{V_{f s}}{V_{m s}}$ \\
\hline
\end{tabular}

Table 2. Space Launch System RBM TDT test section flow conditions in R134a test medium.

\begin{tabular}{|c|c|c|c|c|c|}
\hline $\begin{array}{l}\text { Mach } \\
\text { Number }\end{array}$ & $\mathbf{q}, \mathbf{p s f}$ & $\mathrm{V}, \mathrm{ft} / \mathrm{s}$ & $\mathrm{Re}, / \mathrm{ft}$ & $\begin{array}{l}\text { Re, 1st Stage } \\
\text { Dia. }\end{array}$ & $\begin{array}{l}\text { Model Scale Buffet } \\
\text { Bandwidth,Hz } \\
\text { (Aeroacoustic) }\end{array}$ \\
\hline 0.8 & 481 & 441 & $8.47 \mathrm{E}+06$ & $7.05 \mathrm{E}+06$ & $\begin{array}{c}0-1,002 \\
(0-33,390) \\
\end{array}$ \\
\hline 0.9 & 480 & 499 & $7.47 \mathrm{E}+06$ & $6.22 \mathrm{E}+06$ & $\begin{array}{c}0-1,002 \\
(0-33,388)\end{array}$ \\
\hline 1.0 & 480 & 546 & $6.92 \mathrm{E}+06$ & $5.76 \mathrm{E}+06$ & $\begin{array}{c}0-1,004 \\
(0-33,482) \\
\end{array}$ \\
\hline 1.2 & 300 & 653 & $3.66 \mathrm{E}+06$ & $3.05 \mathrm{E}+06$ & $\begin{array}{c}0-1,059 \\
(0-35,301)\end{array}$ \\
\hline
\end{tabular}


Table 3. Vehicle local alpha and beta for set TDT sting pitch and roll attitude.

\begin{tabular}{|c|c|c|c|c|}
\hline & \multicolumn{2}{|c|}{ Sting Angle; $\theta$, deg } & \\
\hline & & 0.00 & 4.00 & \\
\hline \multirow{6}{*}{ 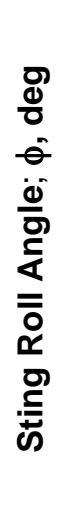 } & \multirow{2}{*}{90.00} & 0.00 & 0.00 & $\alpha$ \\
\hline & & 0.00 & 4.00 & $\boldsymbol{\beta}$ \\
\hline & \multirow{2}{*}{45.00} & 0.00 & 2.83 & $\alpha$ \\
\hline & & 0.00 & 2.83 & $\boldsymbol{\beta}$ \\
\hline & \multirow{2}{*}{0.00} & 0.00 & 4.00 & $\alpha$ \\
\hline & & 0.00 & 0.00 & $\boldsymbol{\beta}$ \\
\hline
\end{tabular}

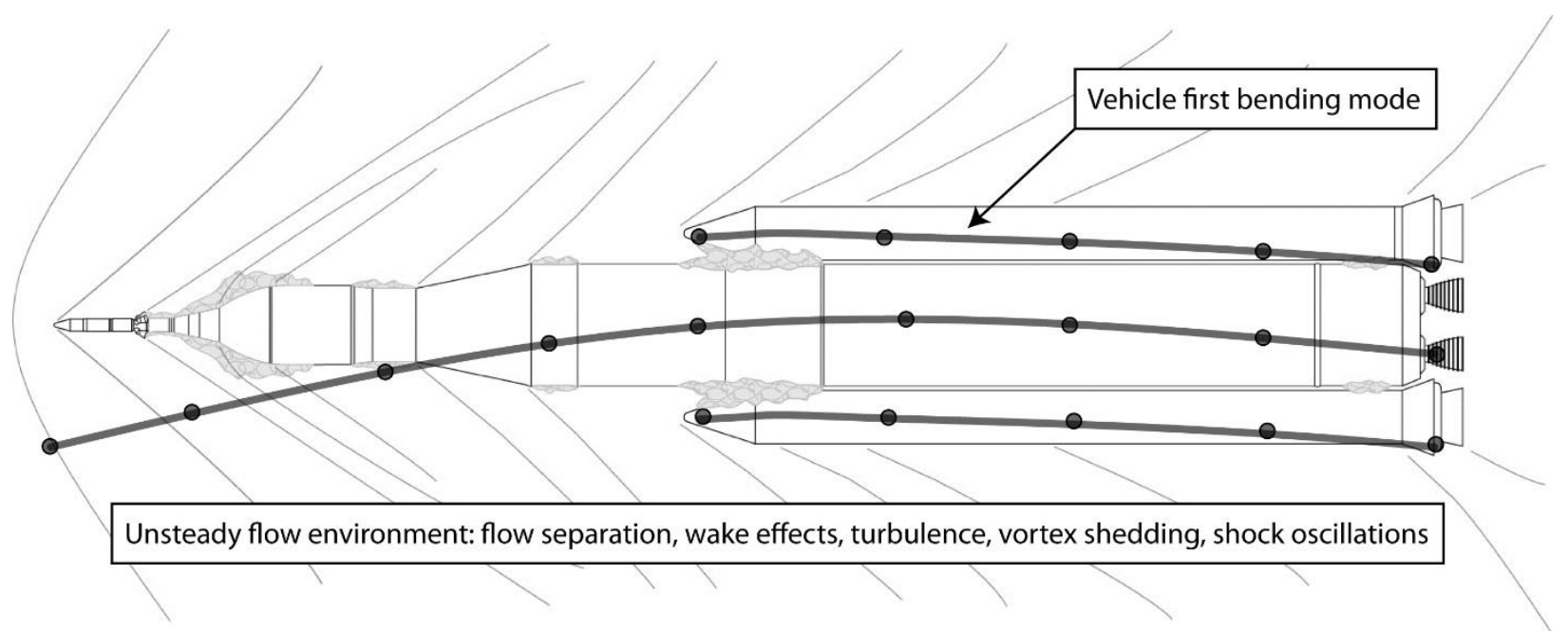

Figure 1. Notional unsteady flow environment and structural deflection for SLS configuration. 


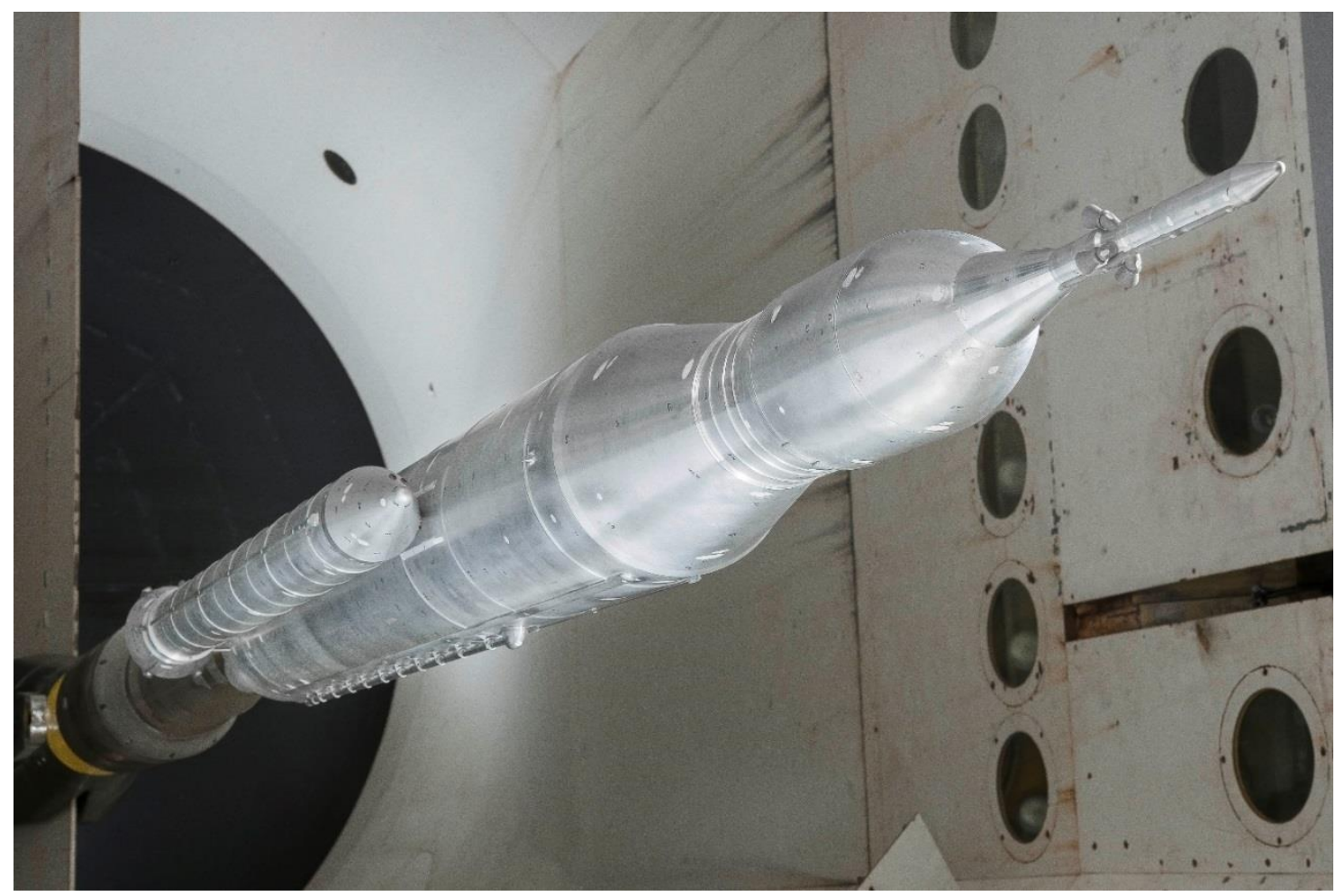

Figure 2. SLS-10005 rigid buffet model installed at TDT.

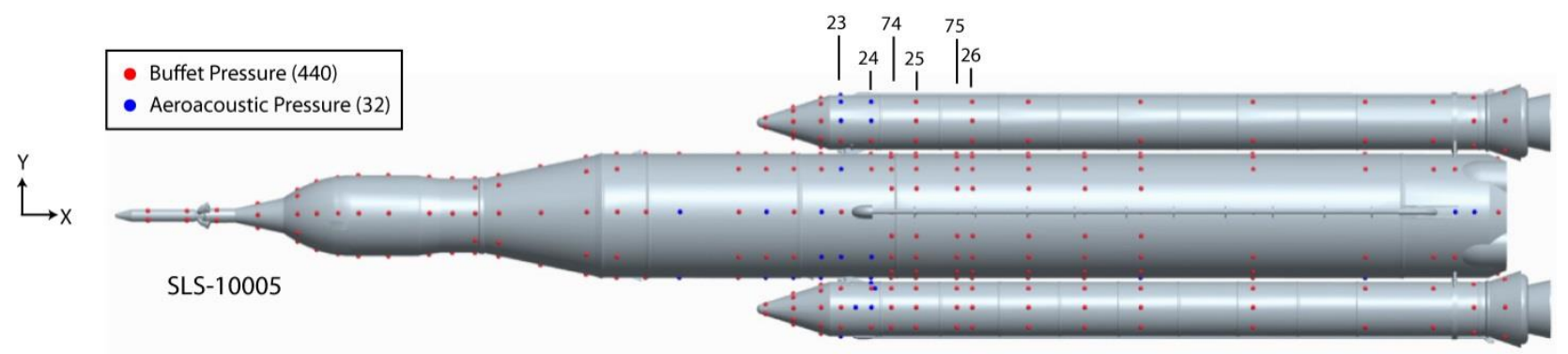

Figure 3. Rigid buffet model transducer locations/stations. 


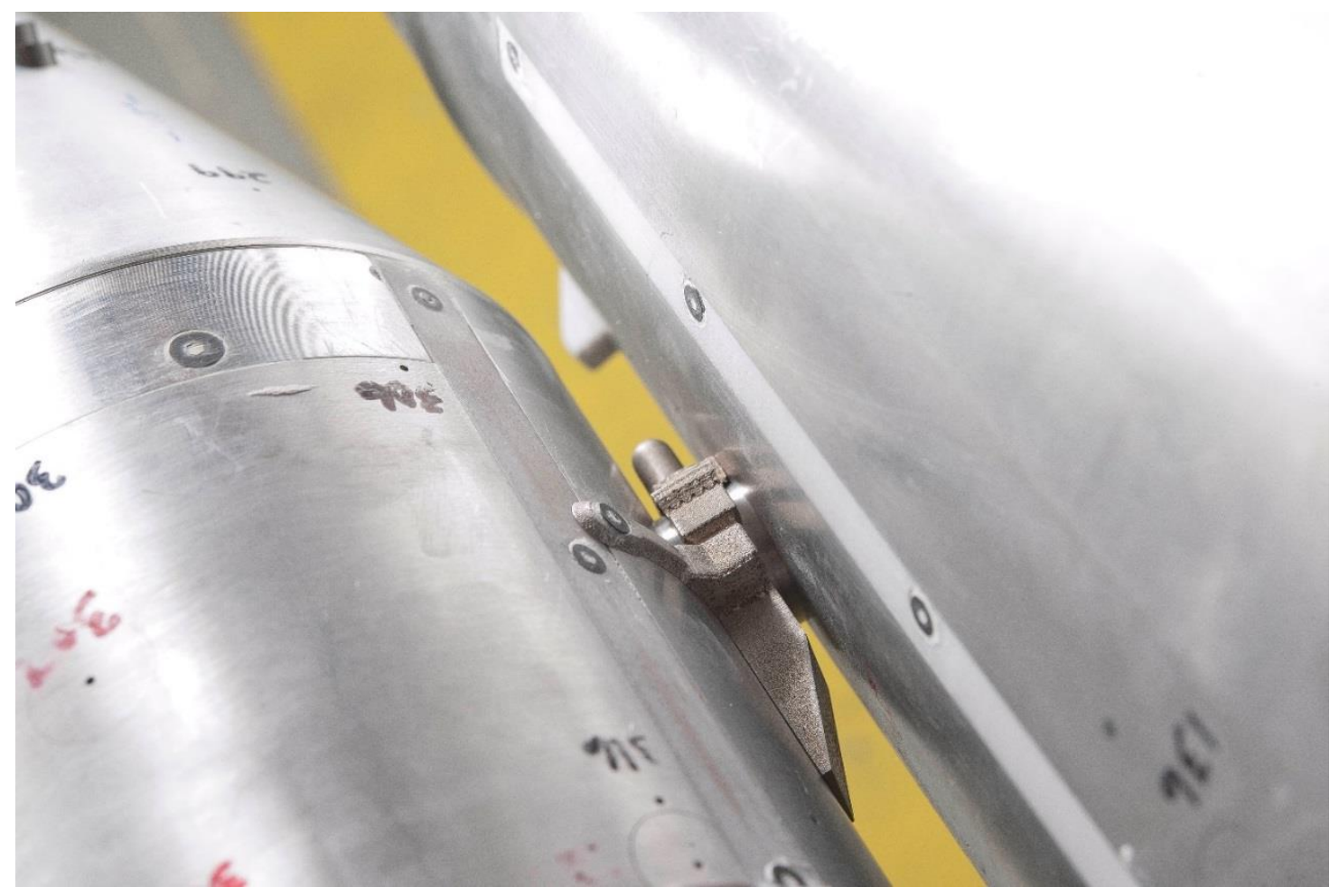

Figure 4. SLS-10005 booster forward attachment protuberance.

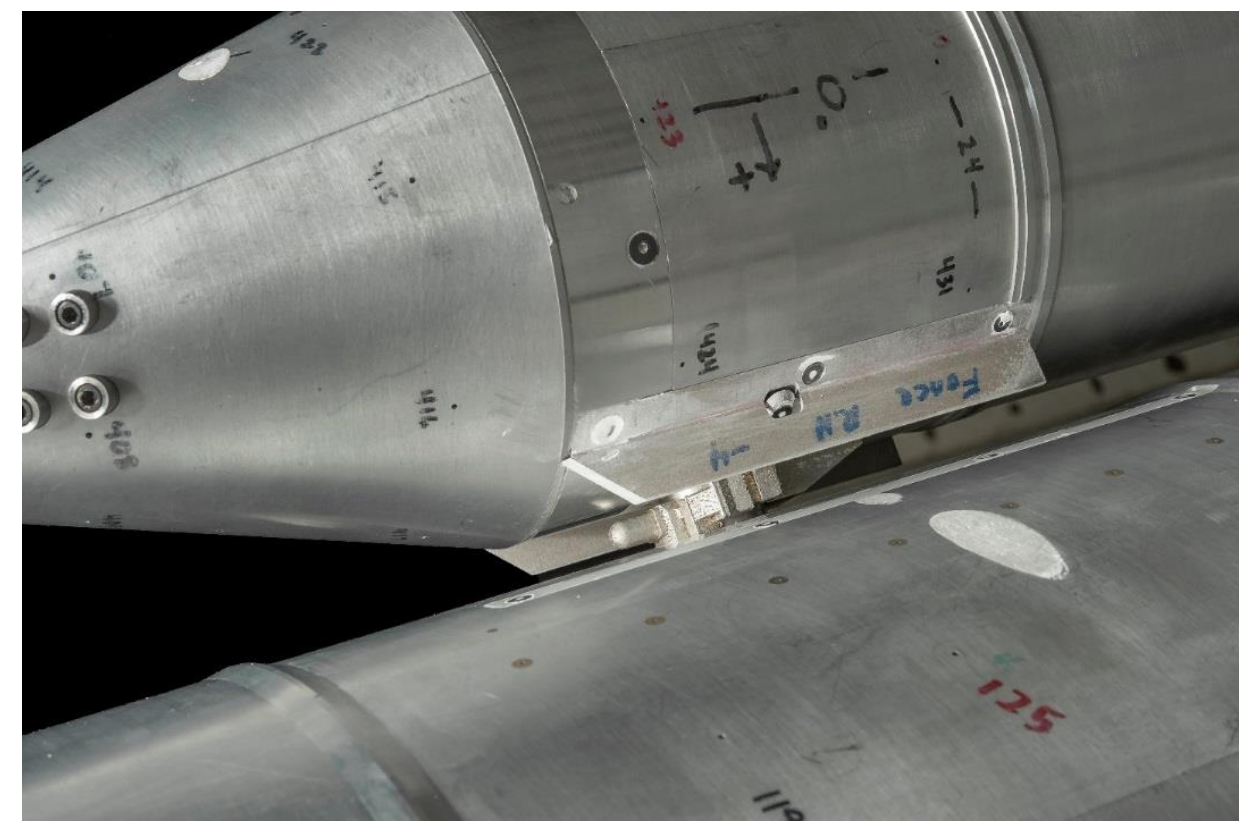

Figure 5. Sharp booster fences. 


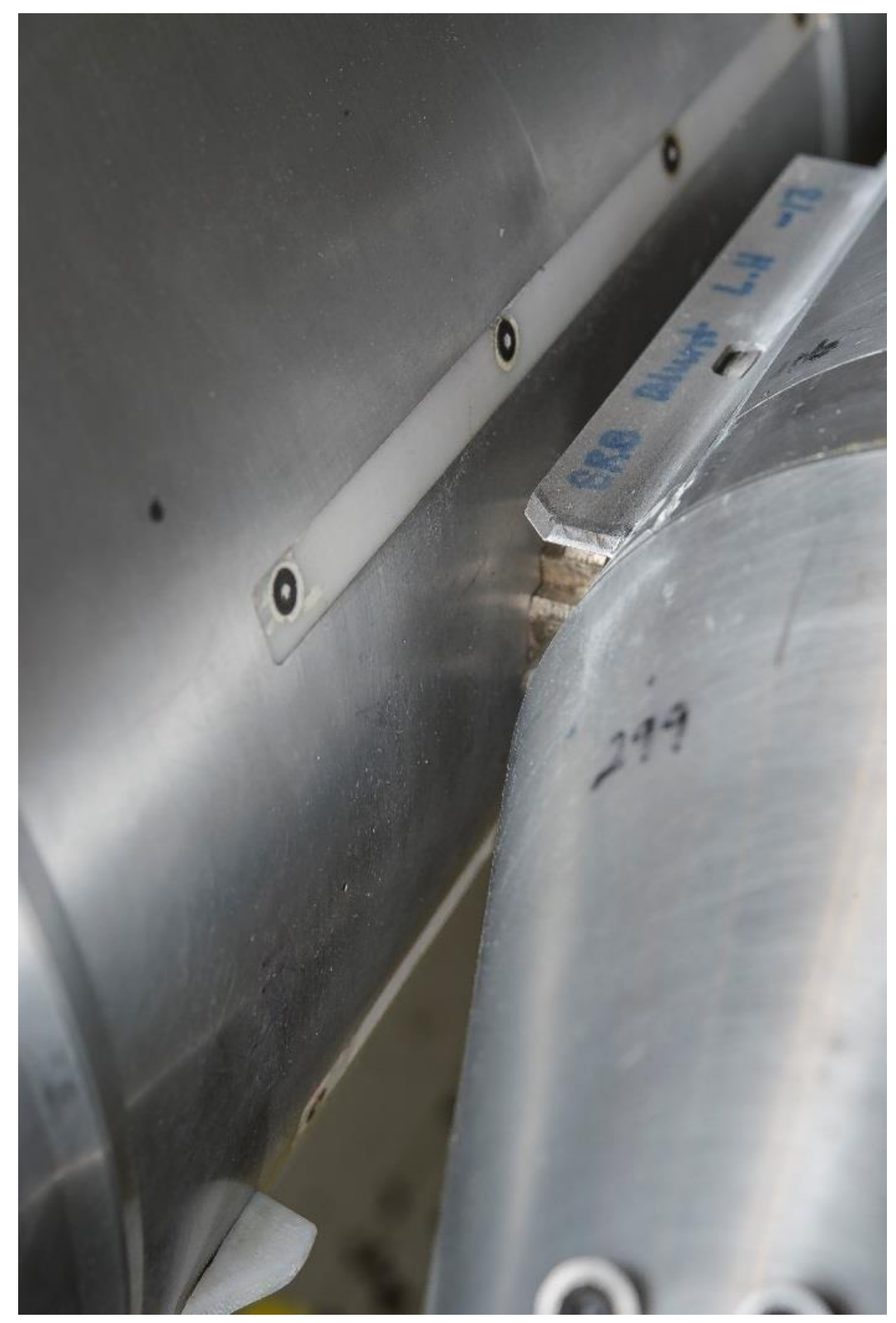

Figure 6. Blunt booster fence (lower fence obscured). 


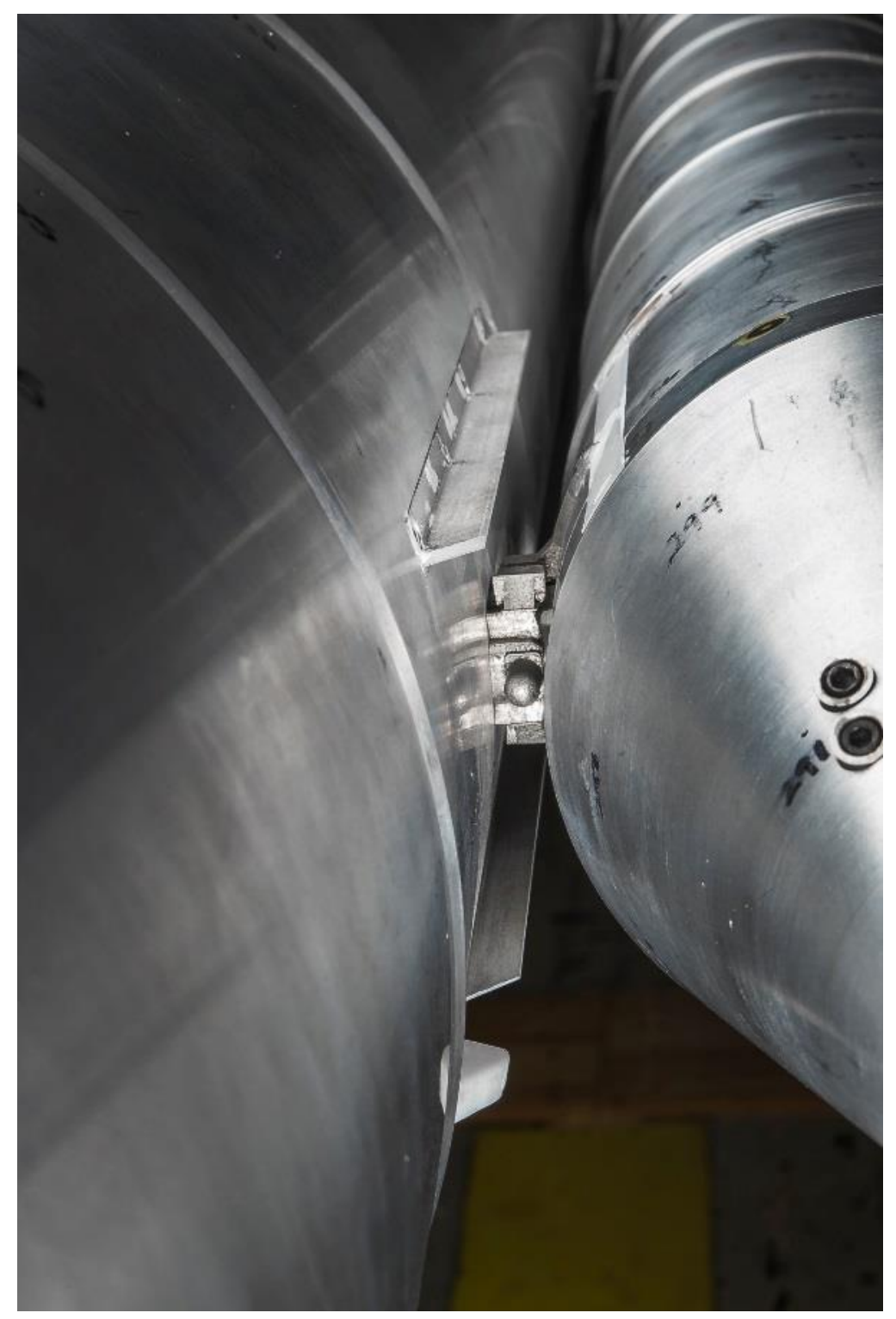

Figure 7. Core fences. 


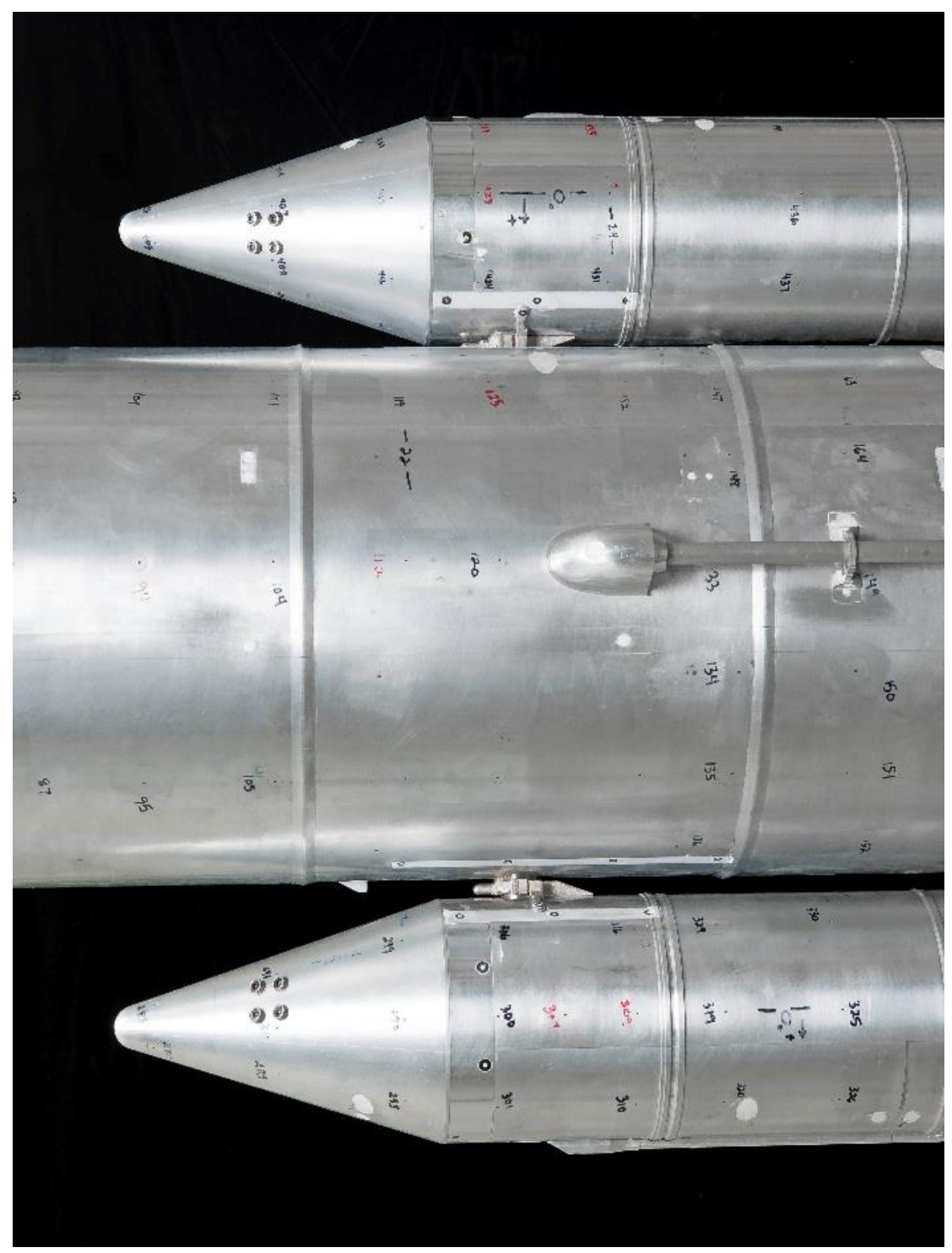

Figure 8. Baseline booster nose cones as viewed from top of model at 0 deg azimuth. 


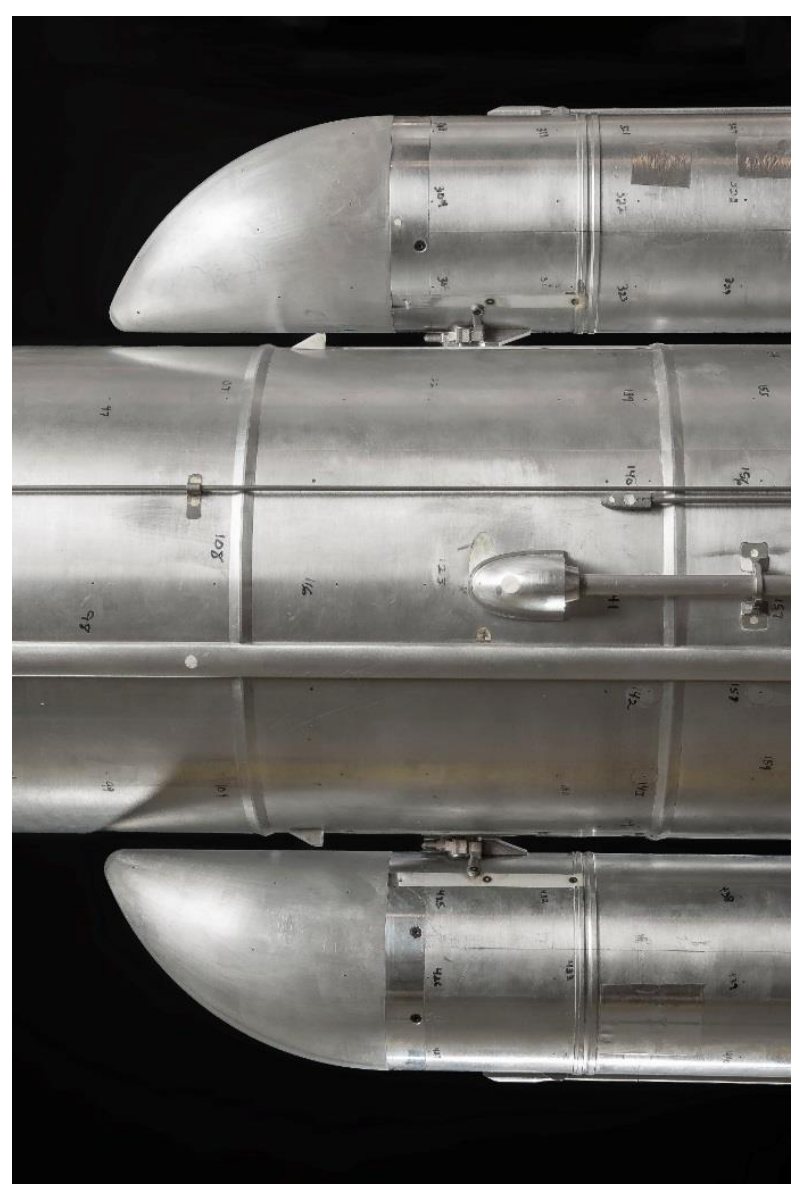

Figure 9. Canted ogive booster nose cones as viewed from bottom of model at 180 deg azimuth.

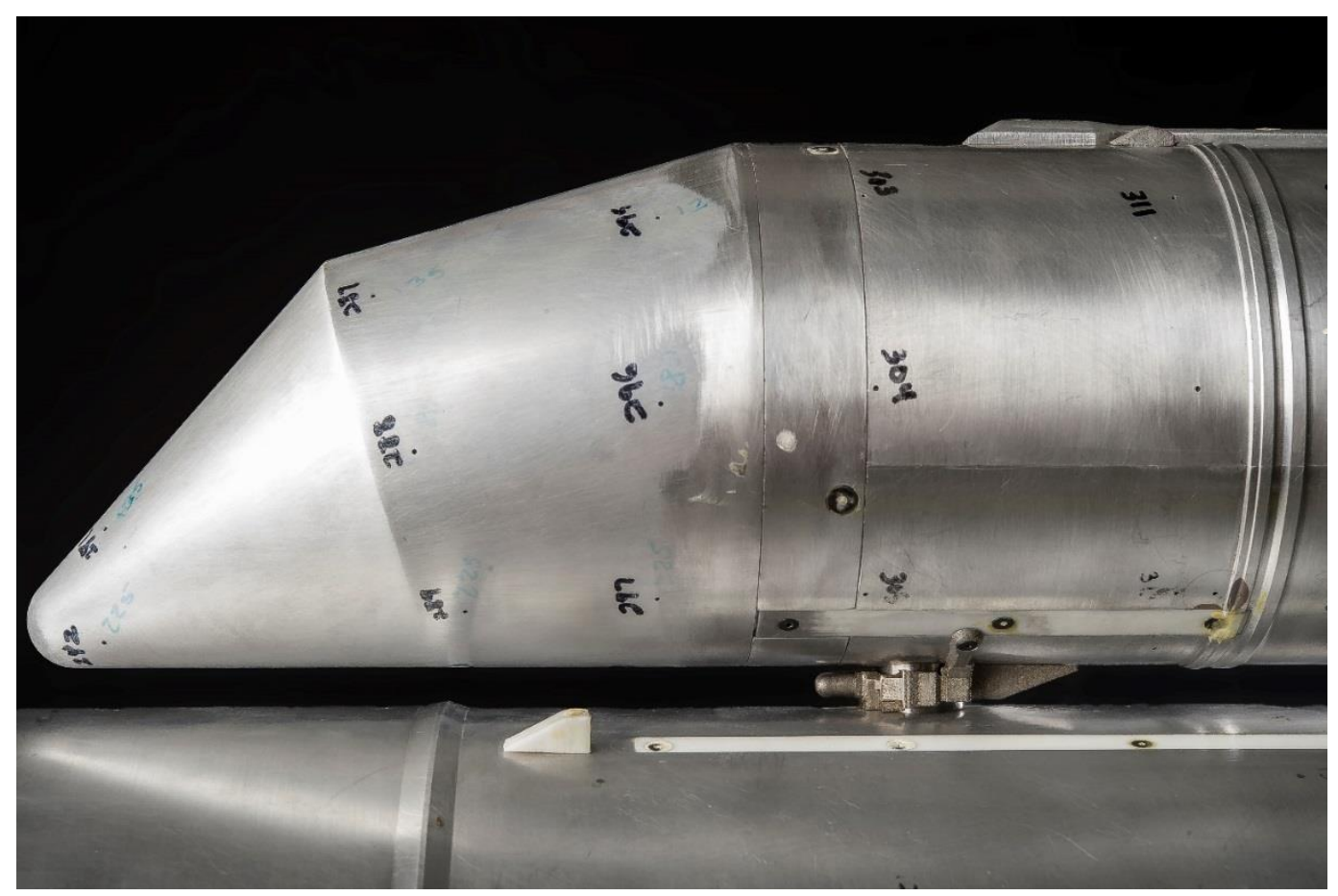

Figure 10. Bent bi-conic nose cone. 


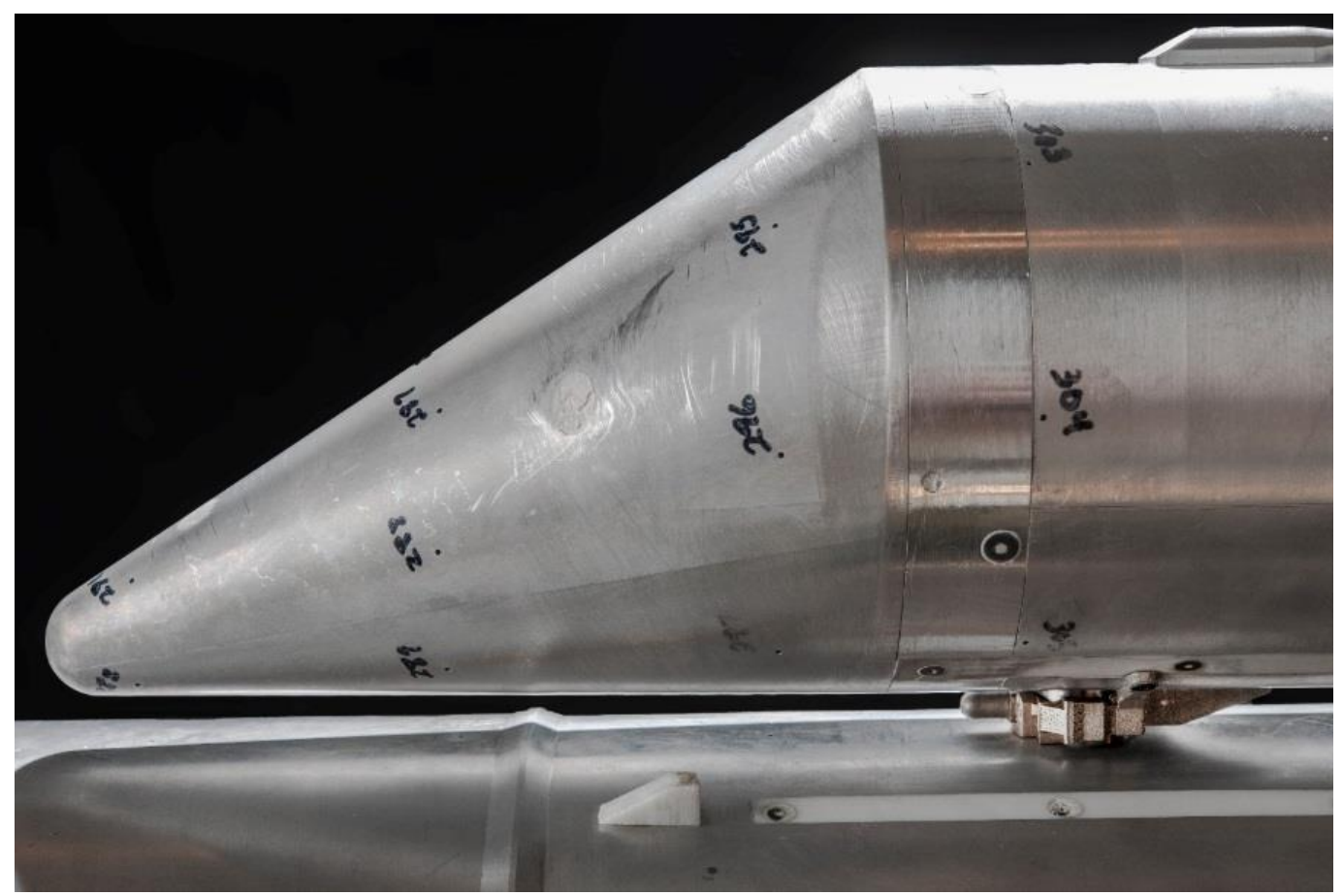

Figure 11. Canted straight nose cone.

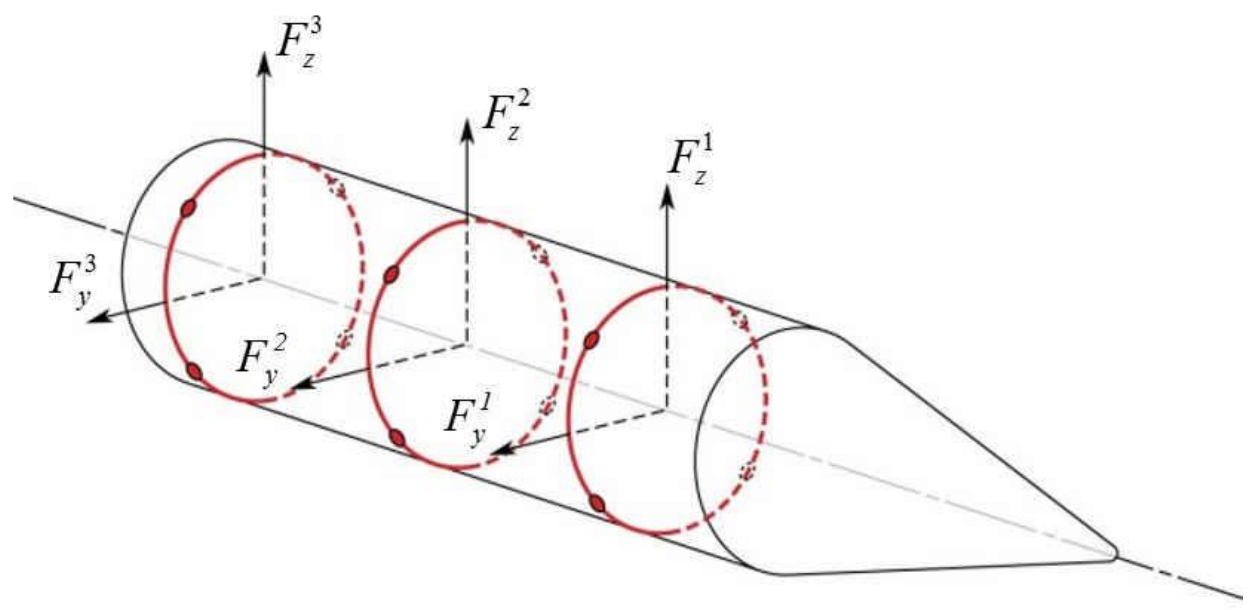

Figure 12. Forcing functions acting on the centerline of vehicle. 


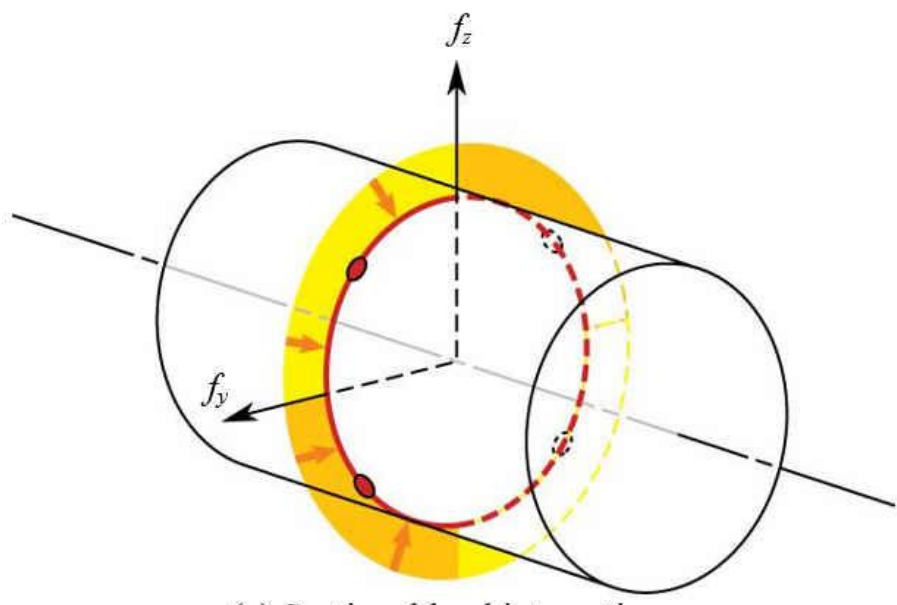

(a) Sectional load integration.

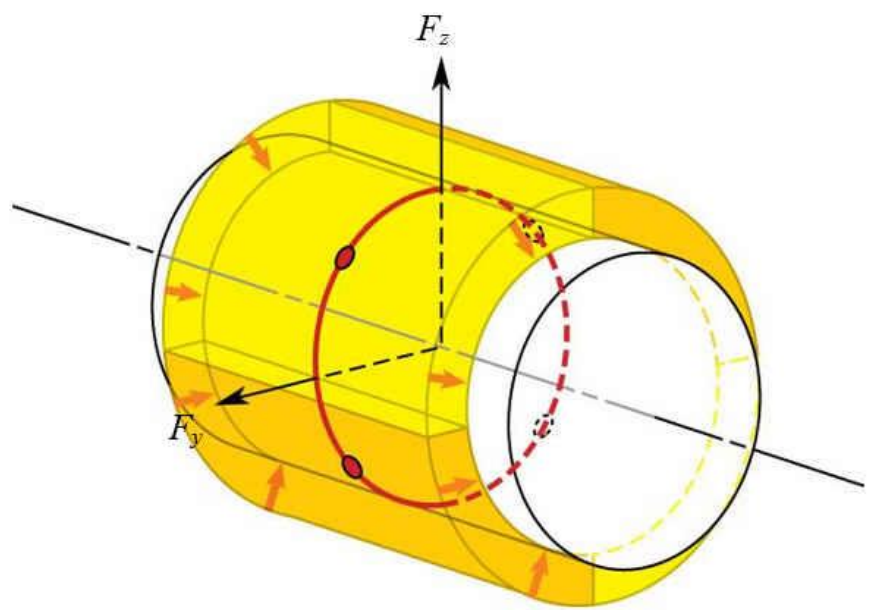

(b) Point load integration

Figure 13. Methods of pressure integration. 

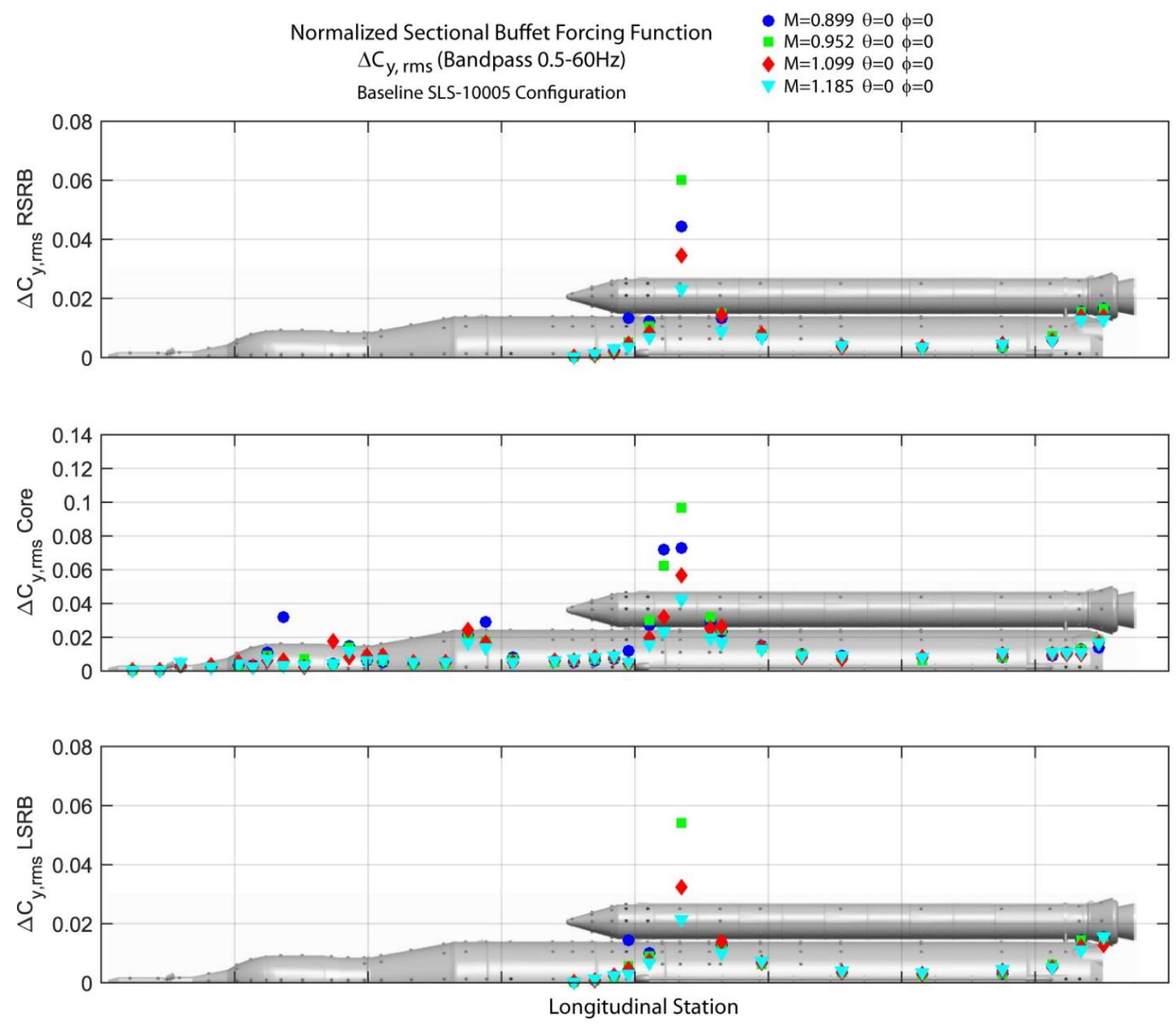

Figure 14. Root-mean-squared trends in y-direction normalized sectional buffet forcing function with increasing Mach number at $\theta=0^{\circ}$ and $\phi=0^{\circ}$ for the baseline SLS-10005 configuration. 

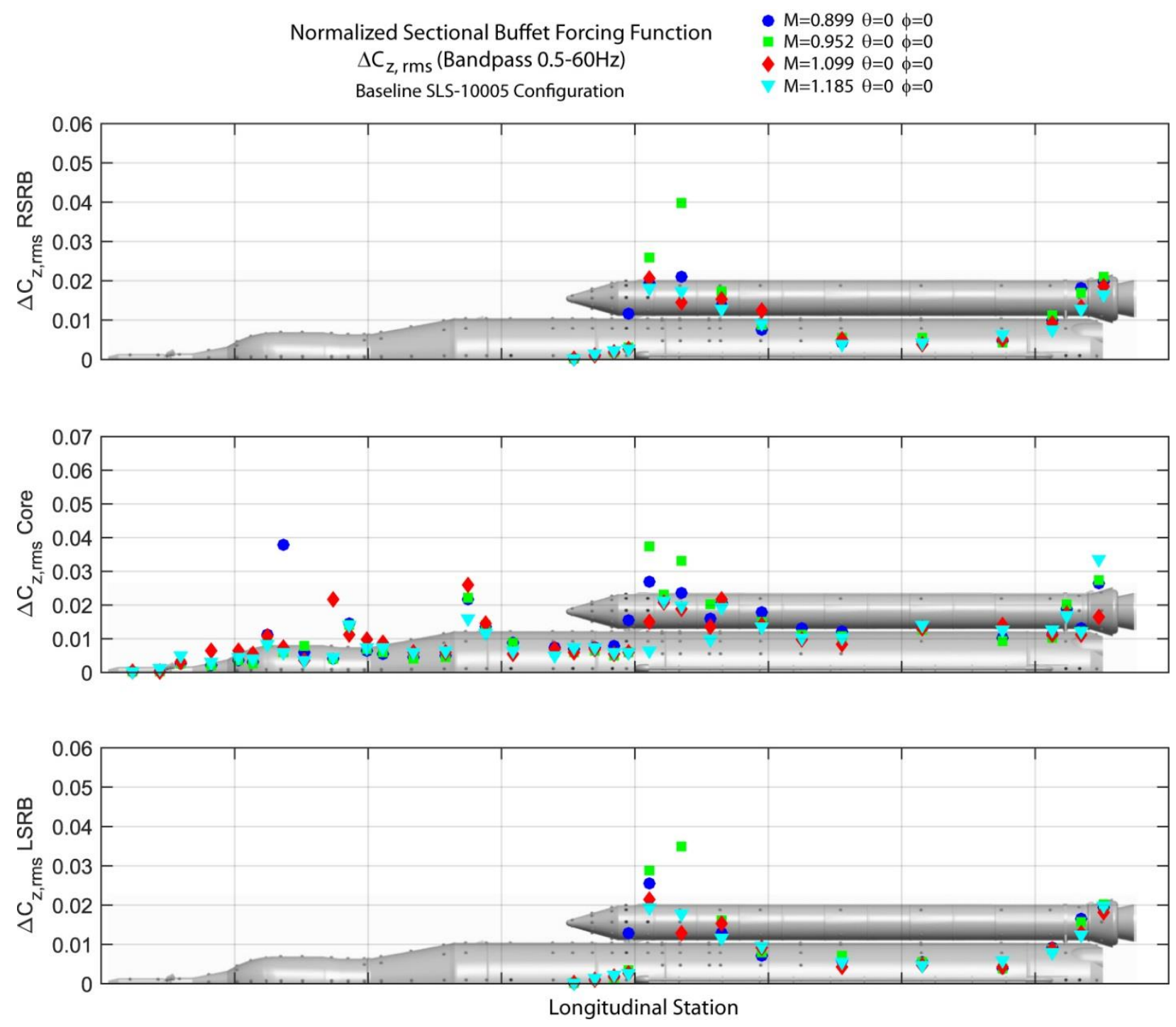

Figure 15. Root-mean-squared trends in z-direction normalized sectional buffet forcing function with increasing Mach number at $\theta=0^{\circ}$ and $\phi=0^{\circ}$ for the baseline SLS-10005 configuration. 

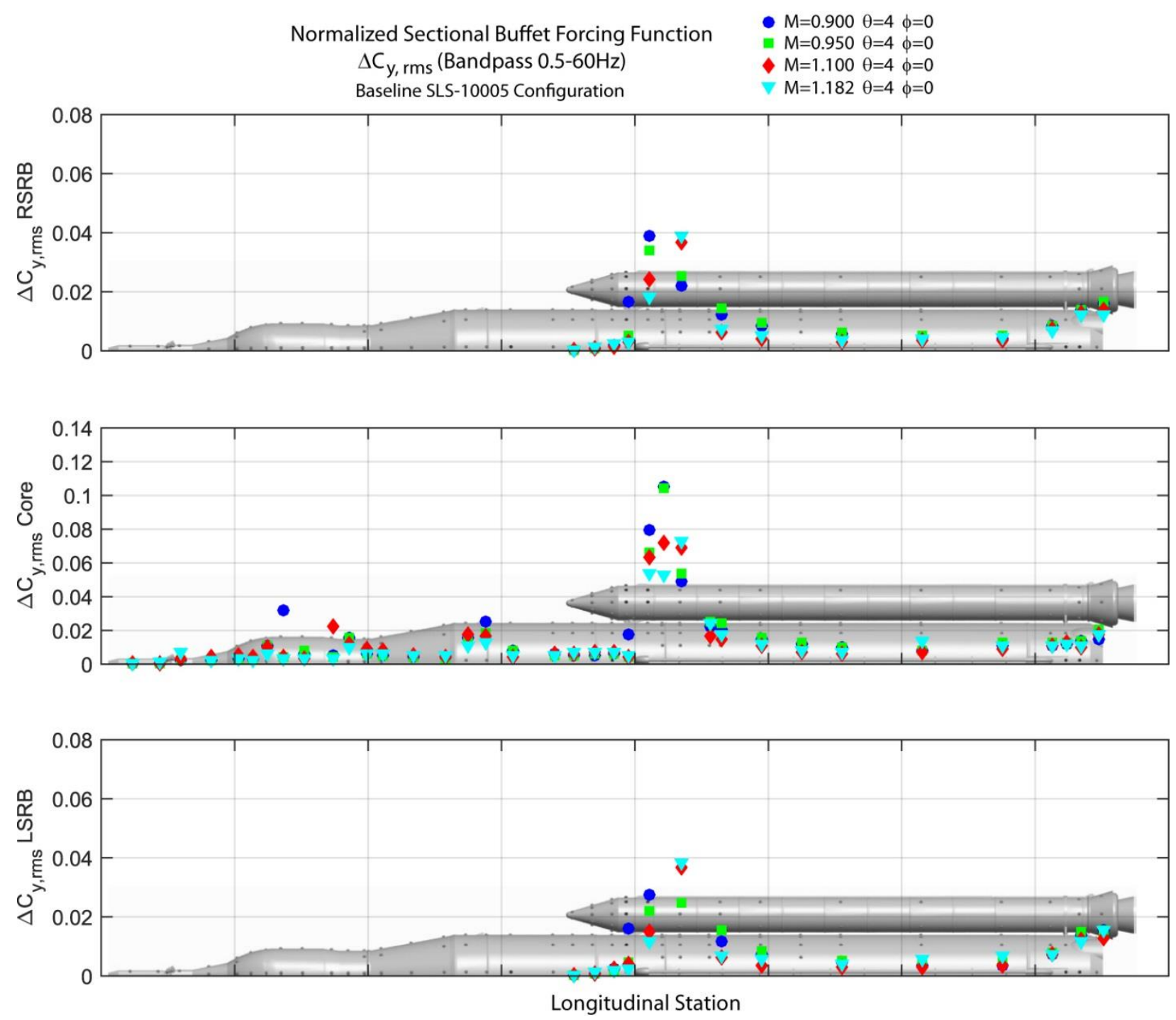

Figure 16. Root-mean-squared trends in y-direction normalized sectional buffet forcing function with increasing Mach number at $\theta=4^{\circ}$ and $\phi=0^{\circ}$ for the baseline SLS-10005 configuration. 

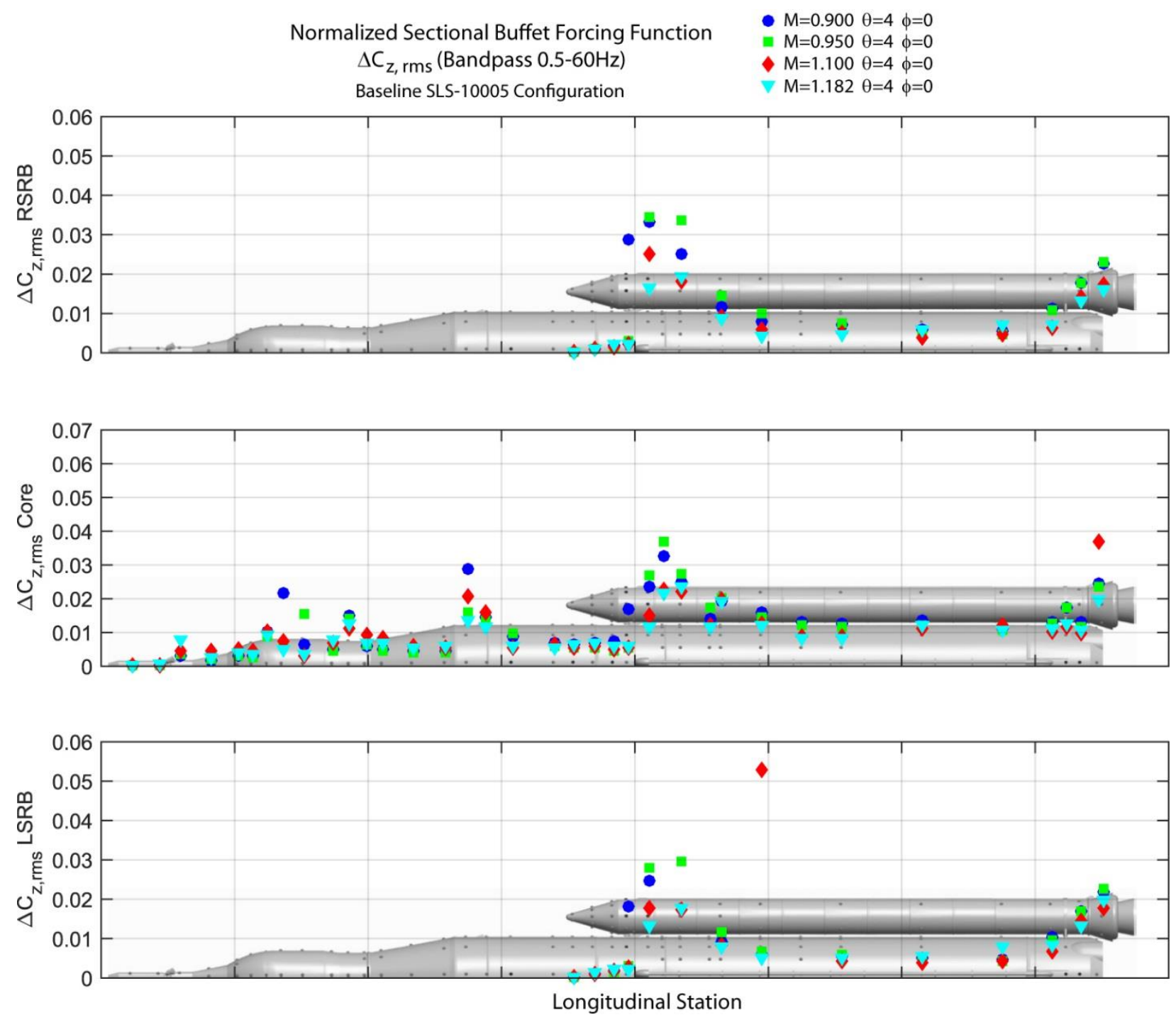

Figure 17. Root-mean-squared trends in z-direction normalized sectional buffet forcing function with increasing Mach number at $\theta=4^{\circ}$ and $\phi=0^{\circ}$ for the baseline SLS-10005 configuration. 

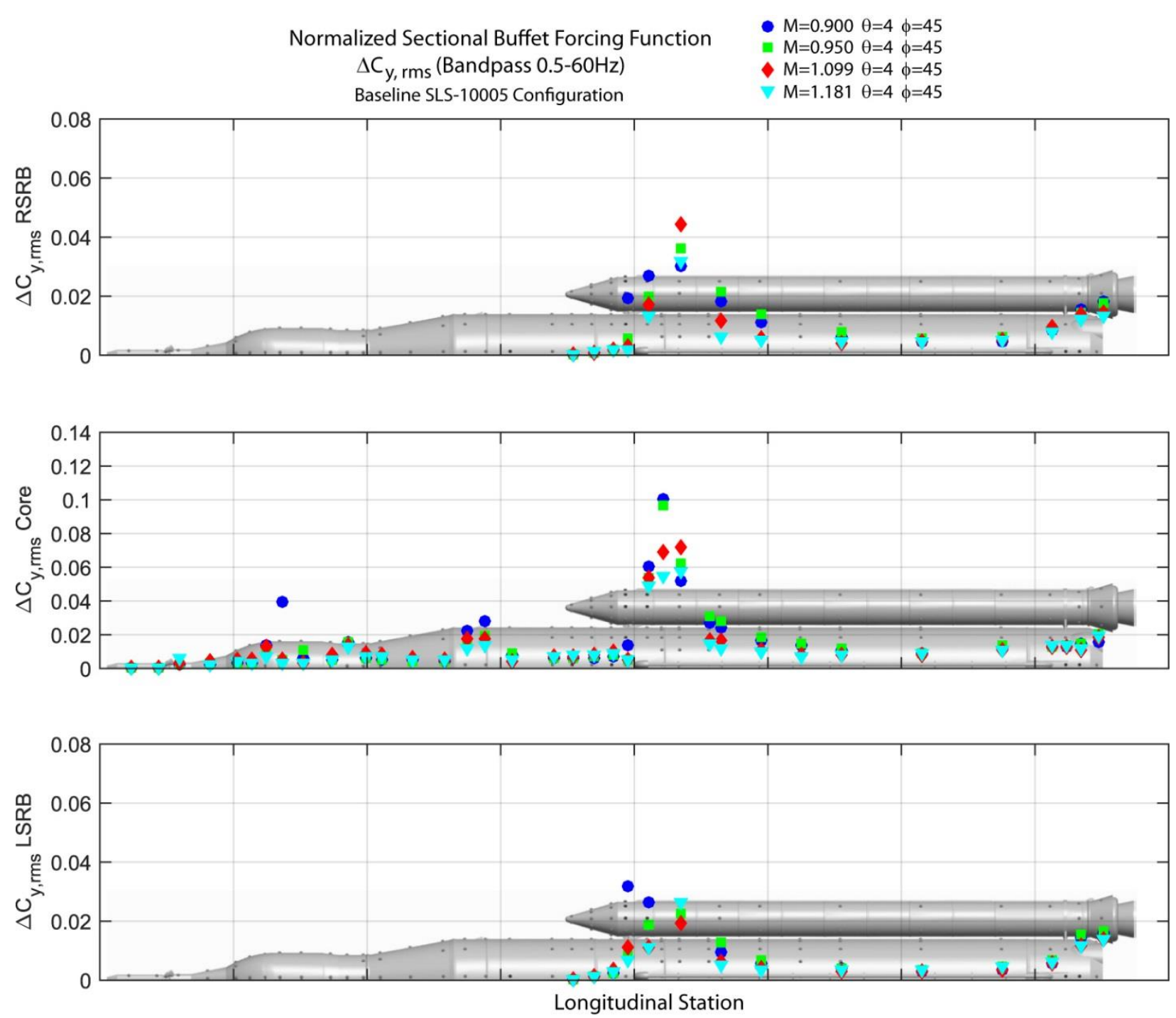

Figure 18. Root-mean-squared trends in y-direction normalized sectional buffet forcing function with increasing Mach number at $\theta=4^{\circ}$ and $\phi=45^{\circ}$ for the baseline SLS-10005 configuration. 

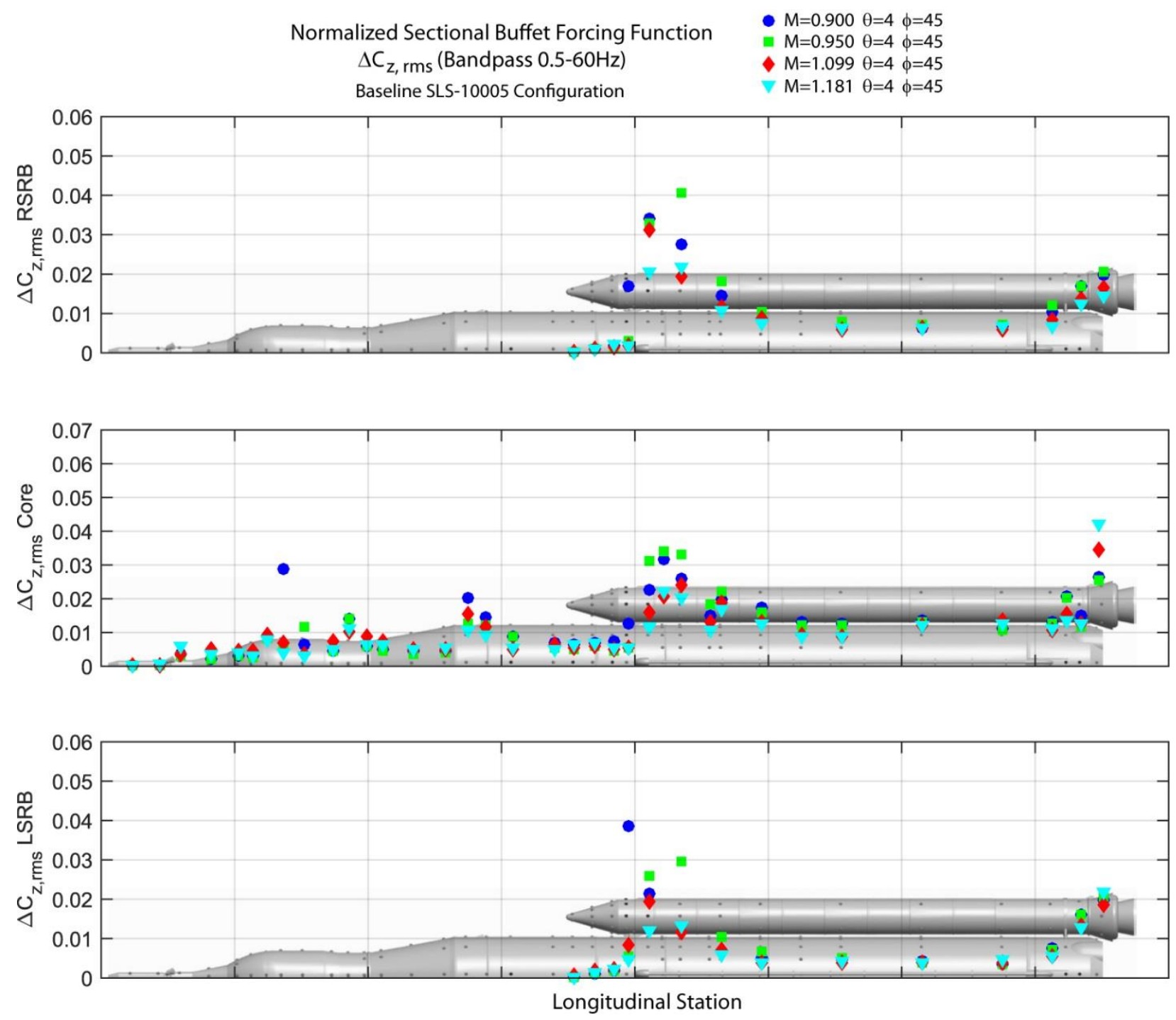

Figure 19. Root-mean-squared trends in z-direction normalized sectional buffet forcing function with increasing Mach number at $\theta=4^{\circ}$ and $\phi=45^{\circ}$ for the baseline SLS- 10005 configuration. 

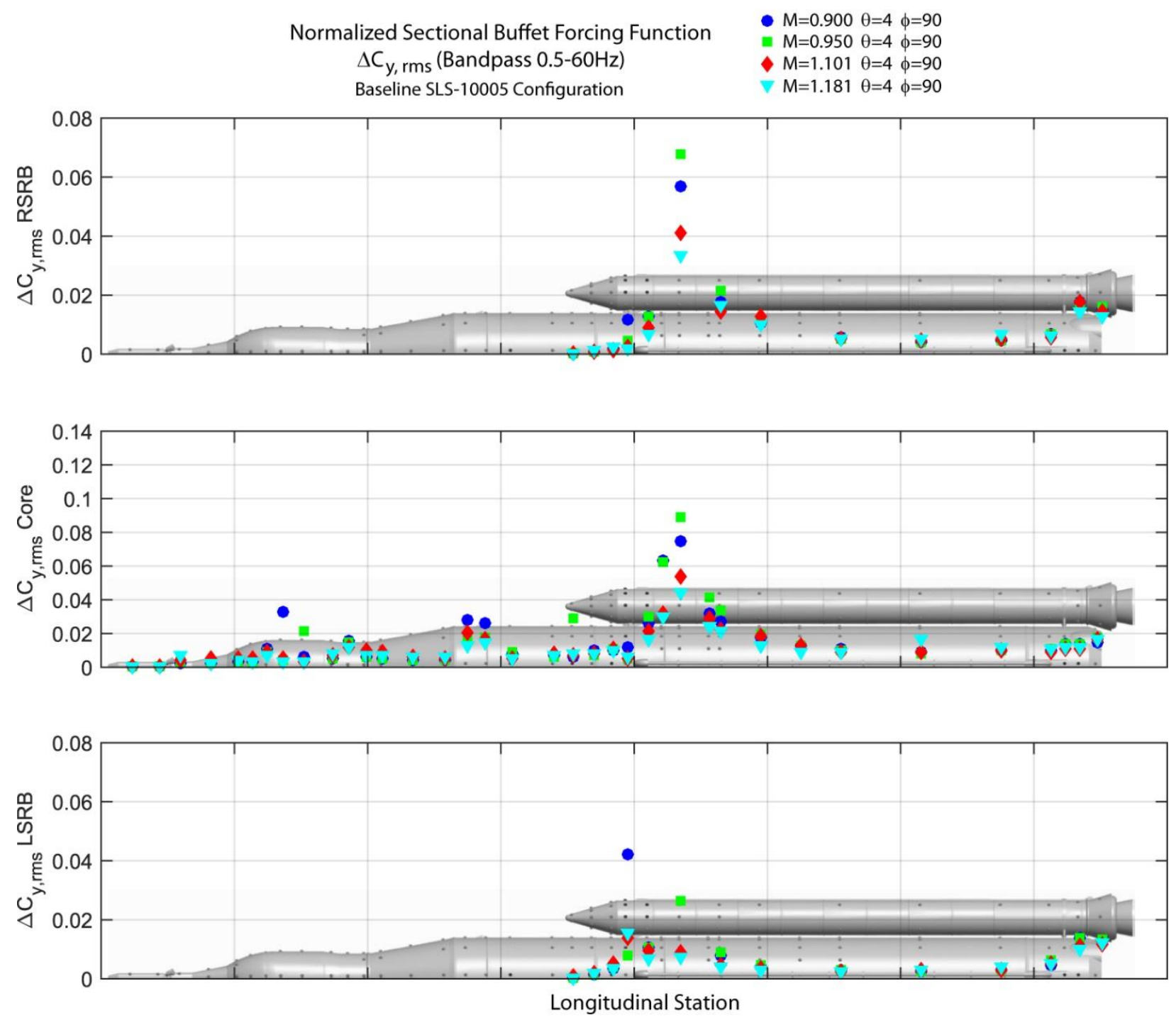

Figure 20. Root-mean-squared trends in y-direction normalized sectional buffet forcing function with increasing Mach number at $\theta=4^{\circ}$ and $\phi=90^{\circ}$ for the baseline SLS-10005 configuration. 

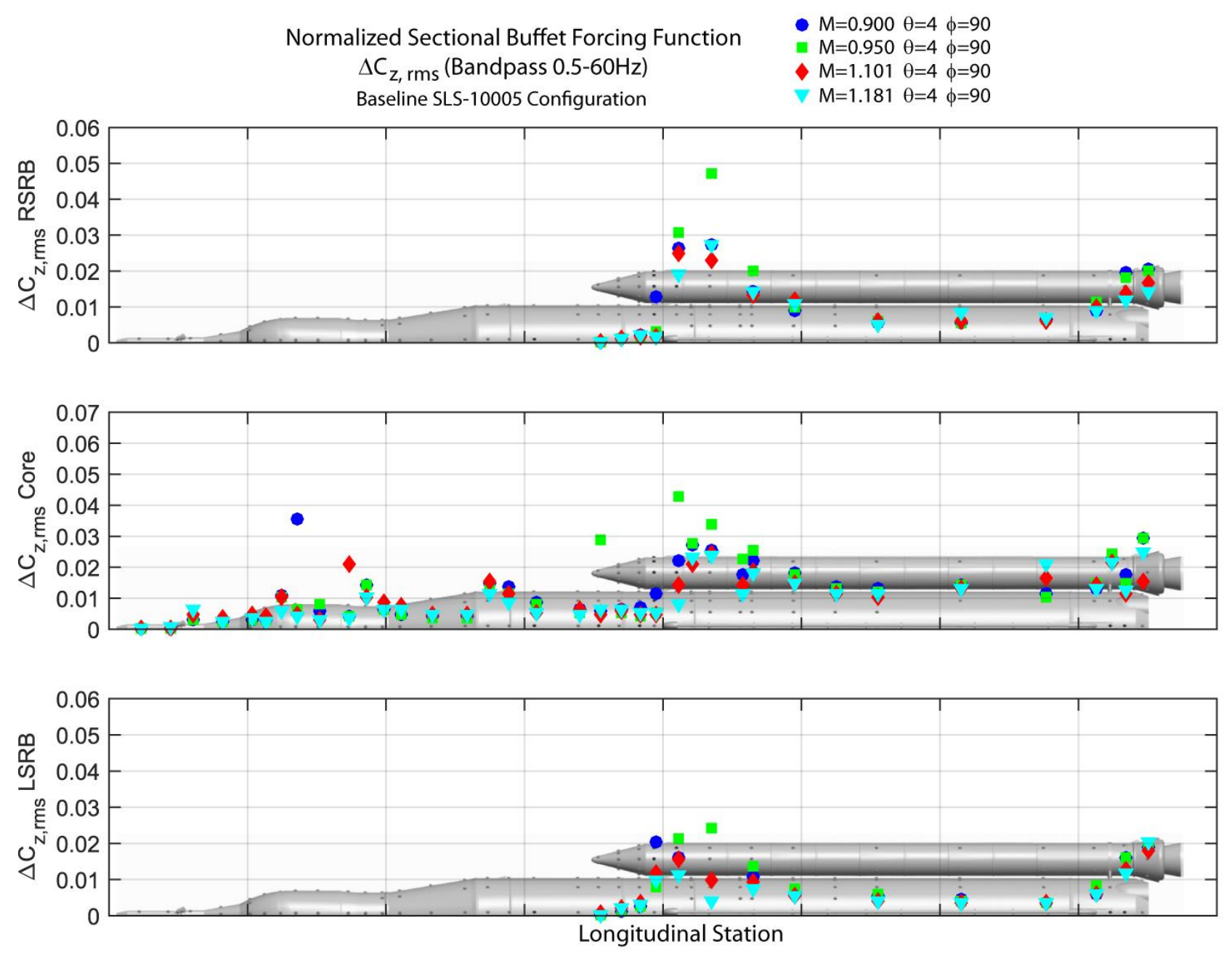

Figure 21. Root-mean-squared trends in z-direction normalized sectional buffet forcing function with increasing Mach number at $\theta=4^{\circ}$ and $\phi=90^{\circ}$ for the baseline SLS-10005 configuration. 

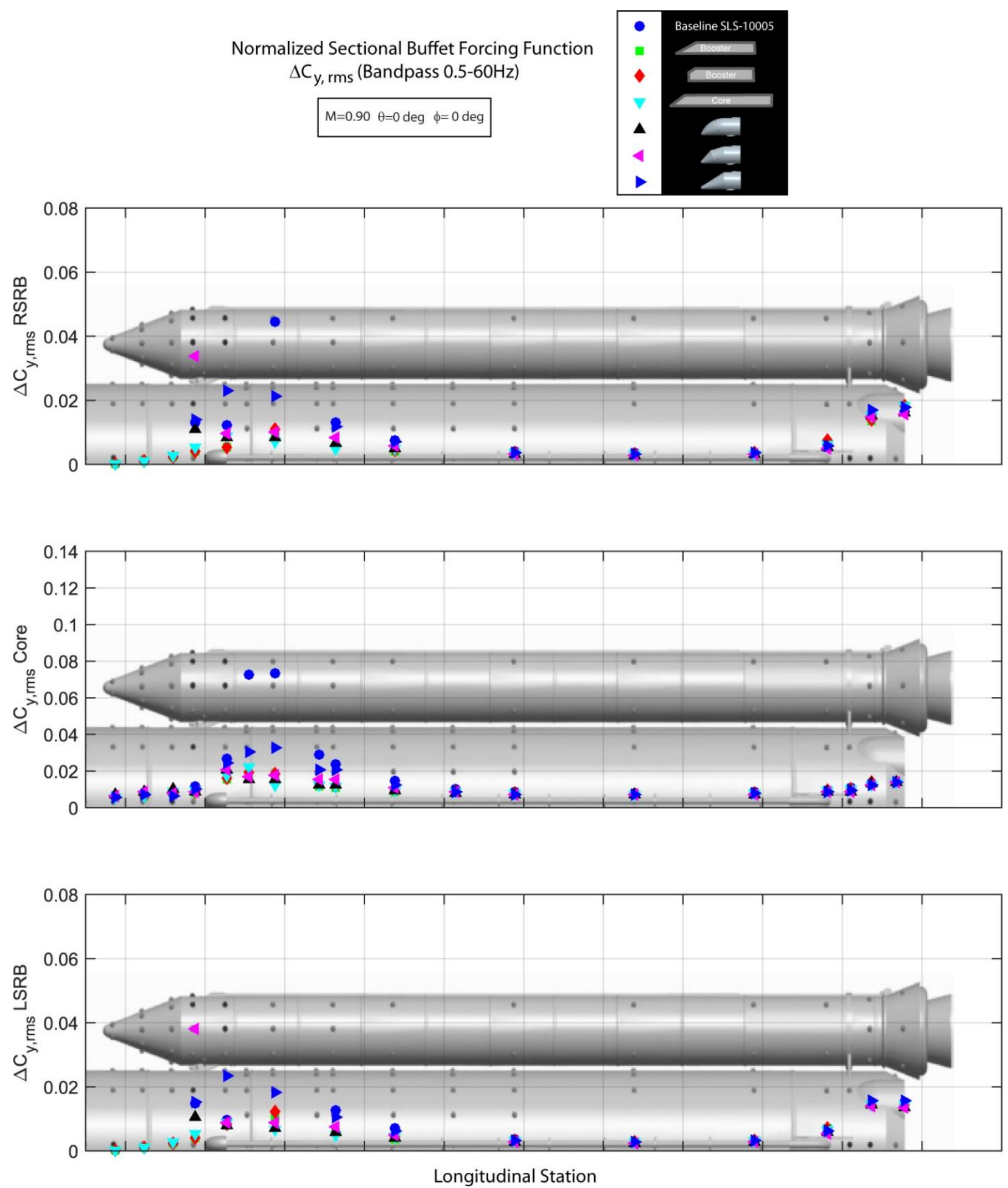

Figure 22. Root-mean-squared trends in y-direction normalized sectional buffet forcing function for baseline and BMO configurations at Mach $0.90, \theta=0^{\circ}$, and $\phi=0^{\circ}$. 

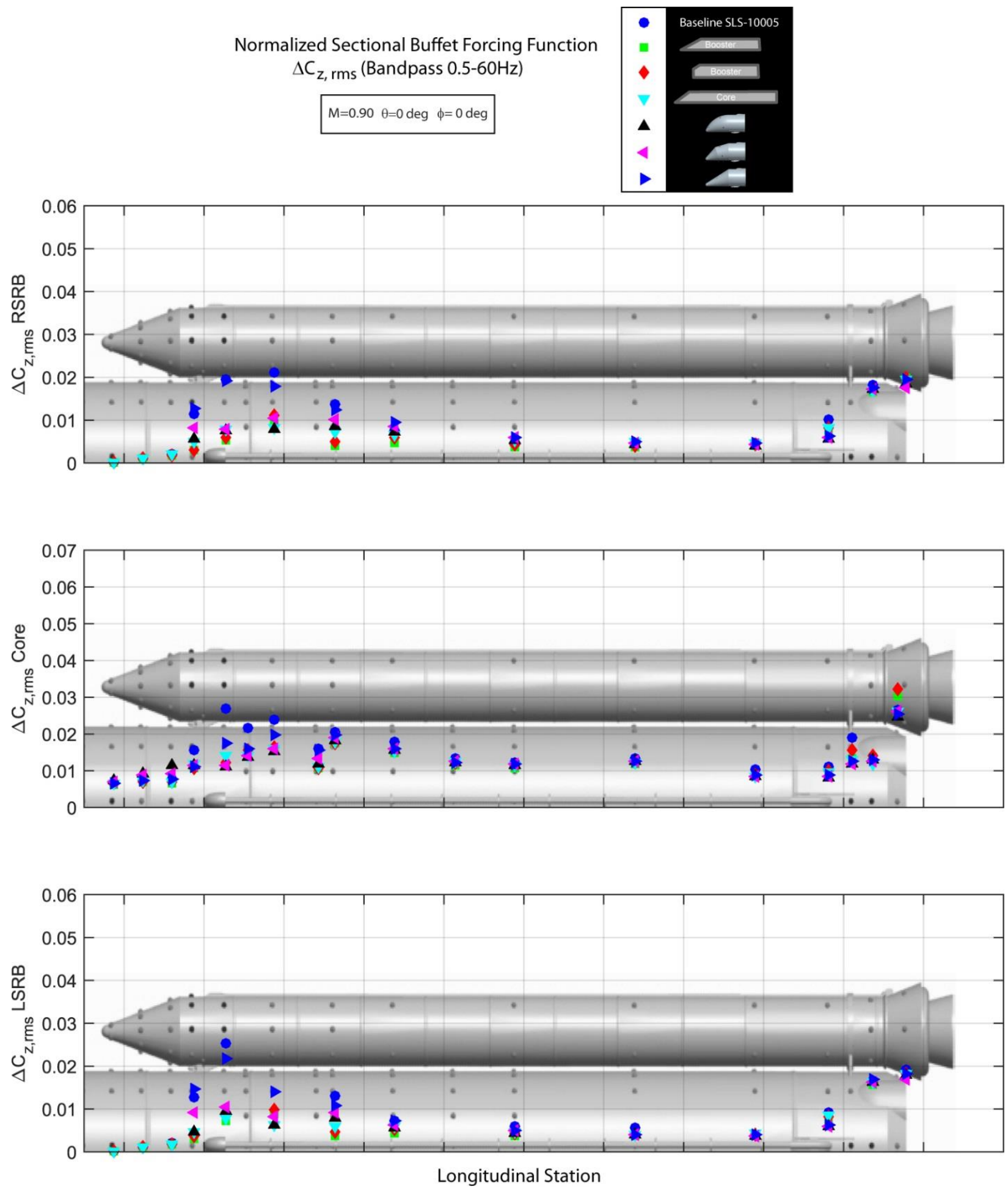

Figure 23. Root-mean-squared trends in z-direction normalized sectional buffet forcing function for baseline and BMO configurations at Mach $0.90, \theta=0^{\circ}$, and $\phi=0^{\circ}$. 

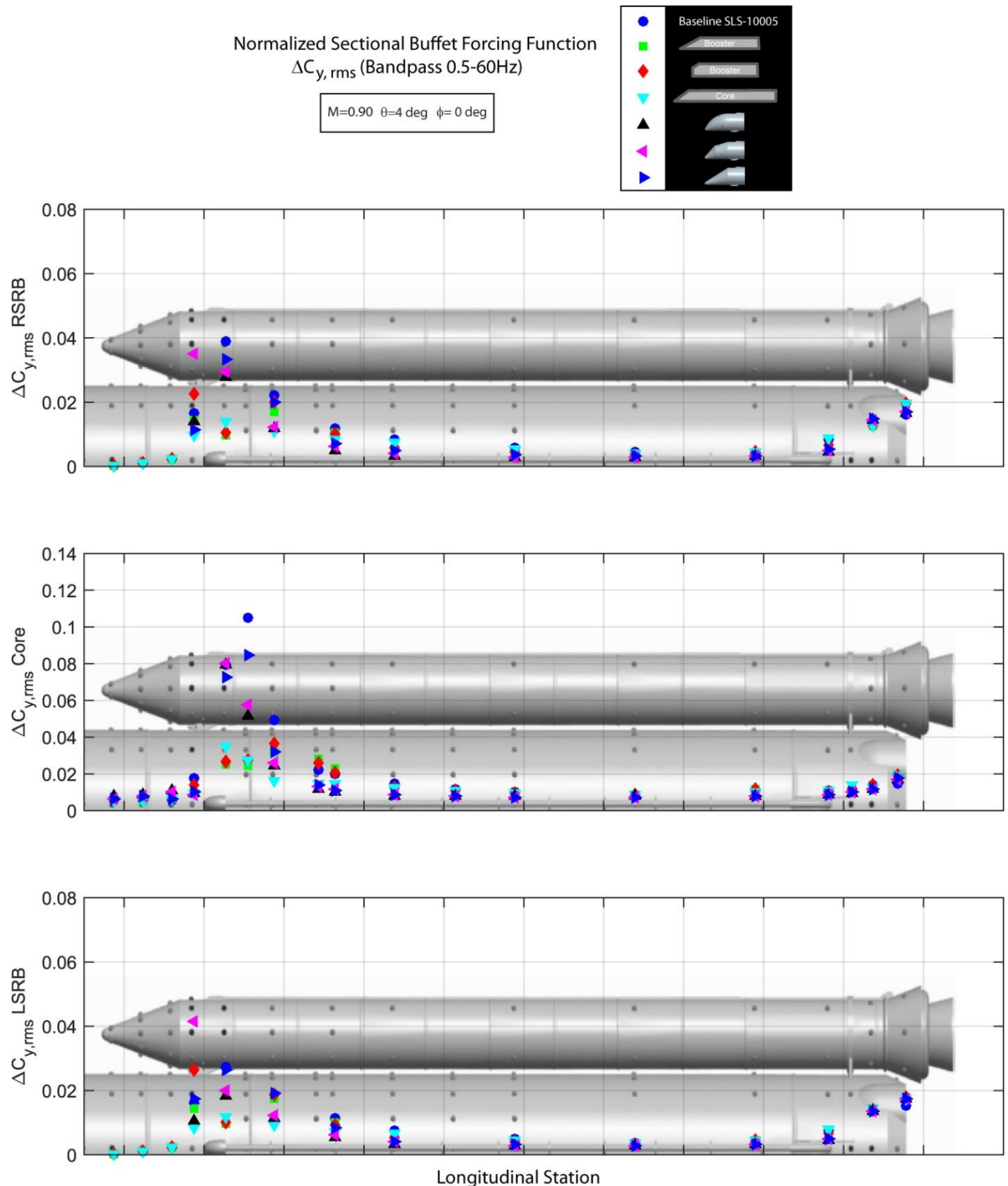

Figure 24. Root-mean-squared trends in y-direction normalized sectional buffet forcing function for baseline and BMO configurations at Mach $0.90, \theta=4^{\circ}$, and $\phi=0^{\circ}$. 

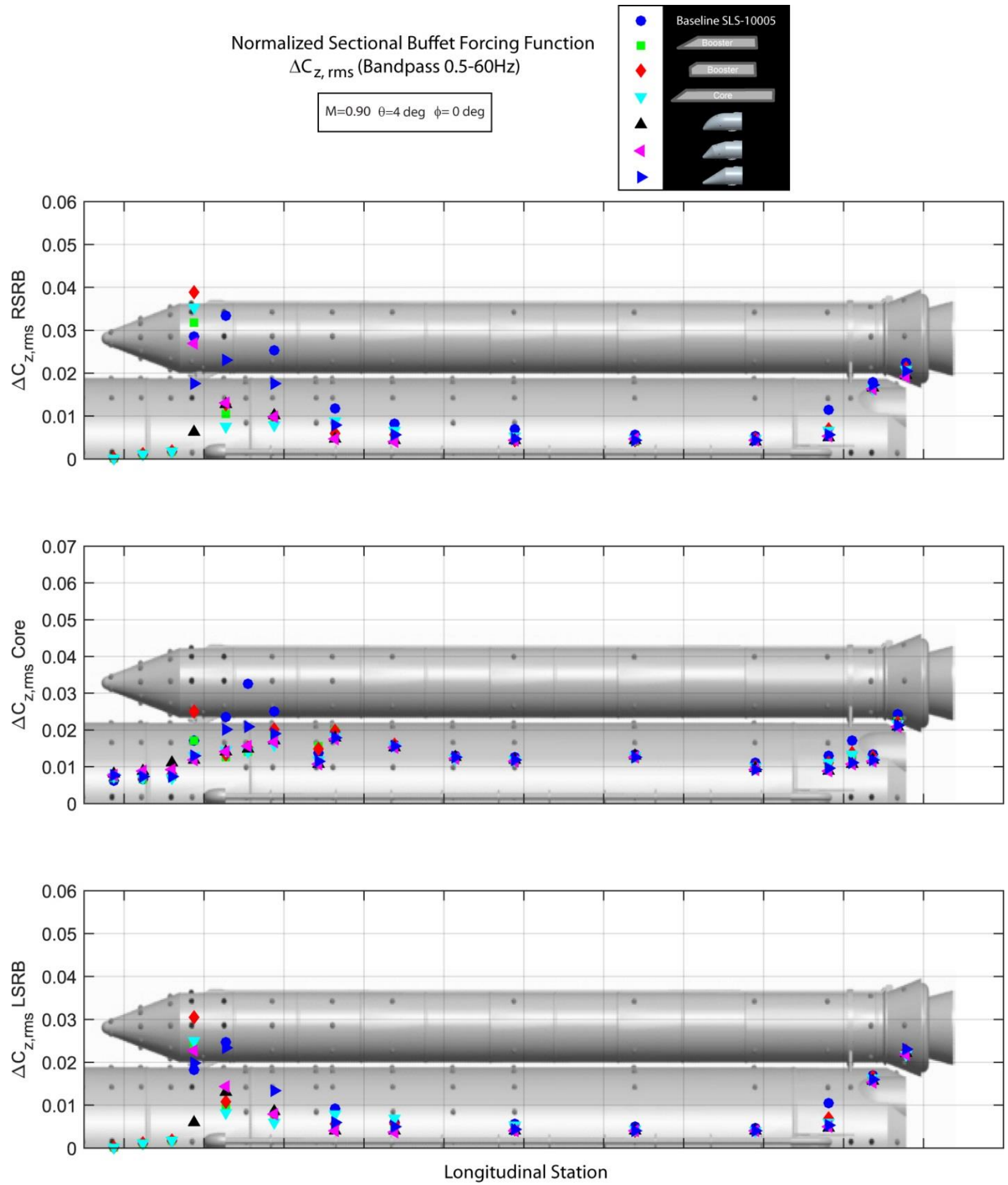

Figure 25. Root-mean-squared trends in z-direction normalized sectional buffet forcing function for baseline and BMO configurations at Mach $0.90, \theta=4^{\circ}$, and $\phi=0^{\circ}$. 

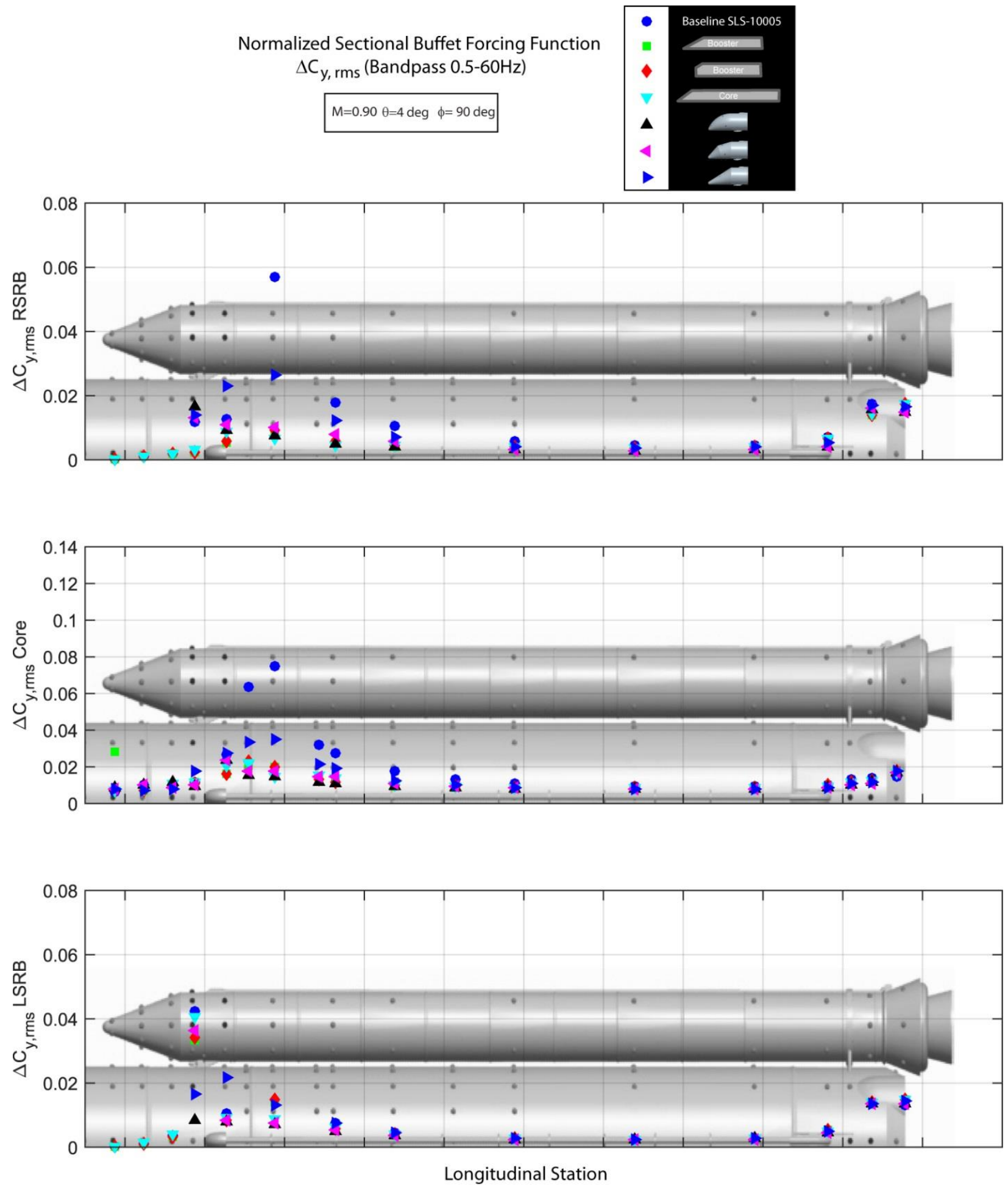

Figure 26. Root-mean-squared trends in y-direction normalized sectional buffet forcing function for baseline and BMO configurations at Mach $0.90, \theta=4^{\circ}$, and $\phi=90^{\circ}$. 

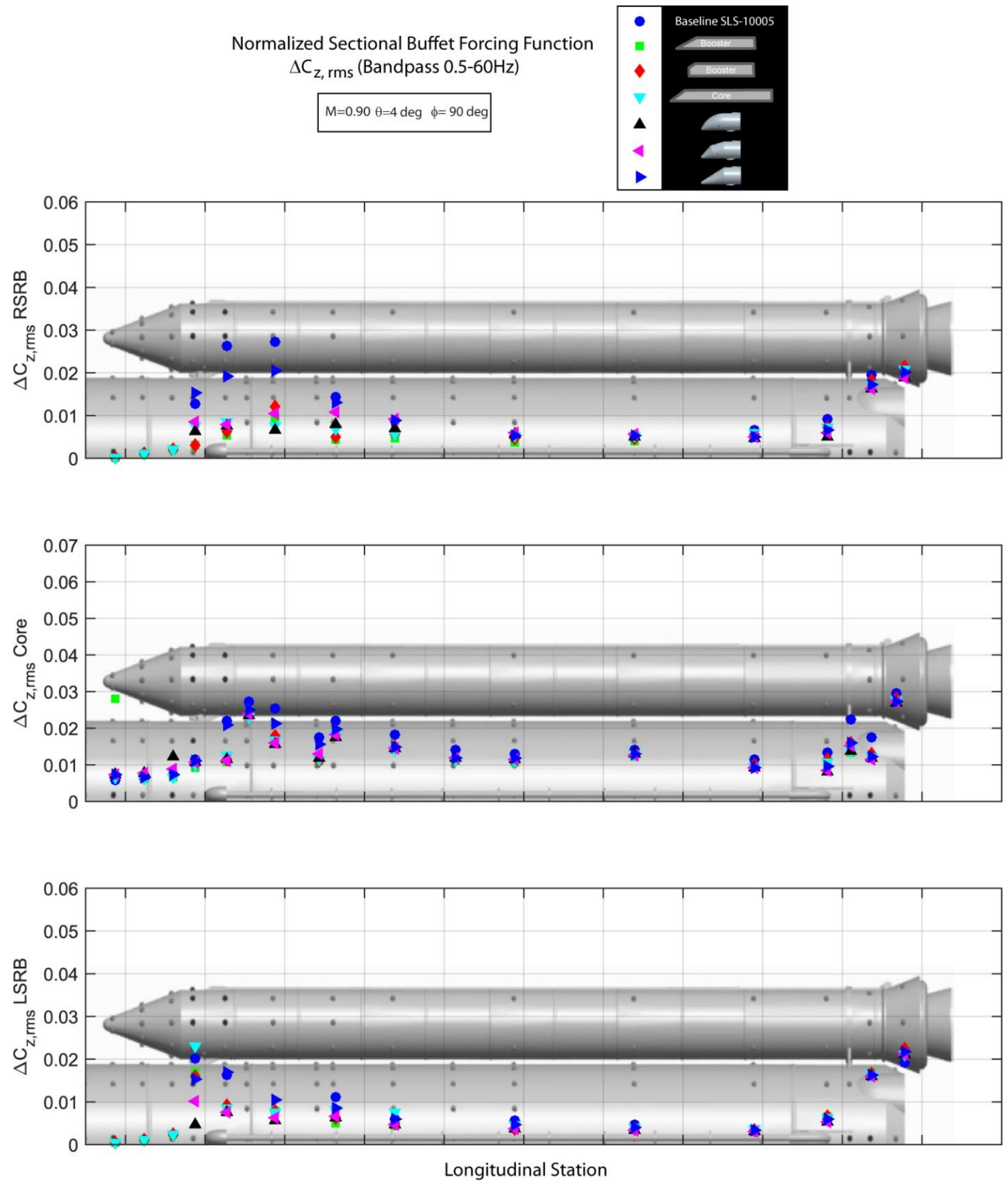

Figure 27. Root-mean-squared trends in z-direction normalized sectional buffet forcing function for baseline and BMO configurations at Mach $0.90, \theta=4^{\circ}$, and $\phi=90^{\circ}$. 

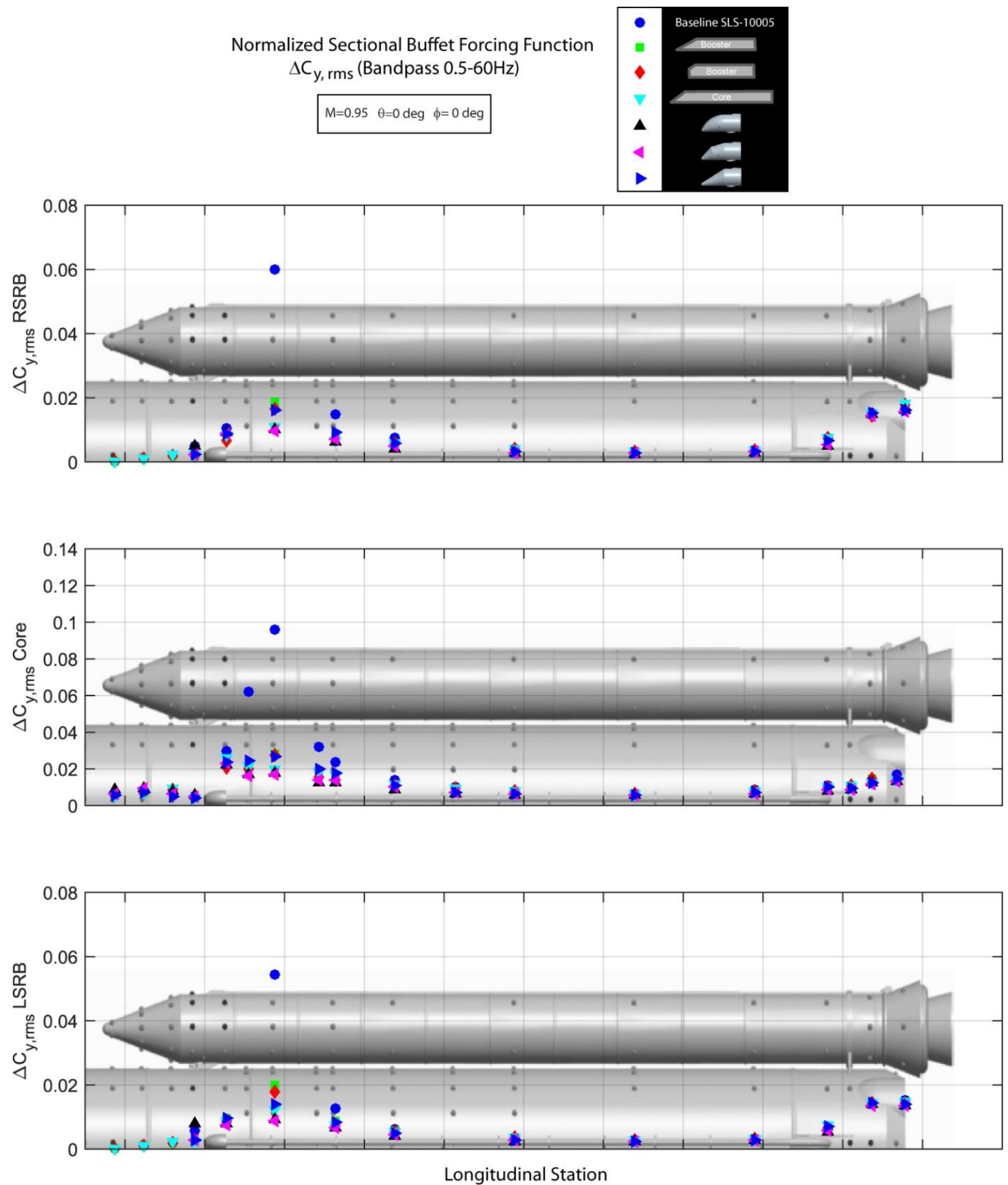

Figure 28. Root-mean-squared trends in y-direction normalized sectional buffet forcing function for baseline and BMO configurations at Mach $0.95, \theta=0^{\circ}$, and $\phi=0^{\circ}$. 

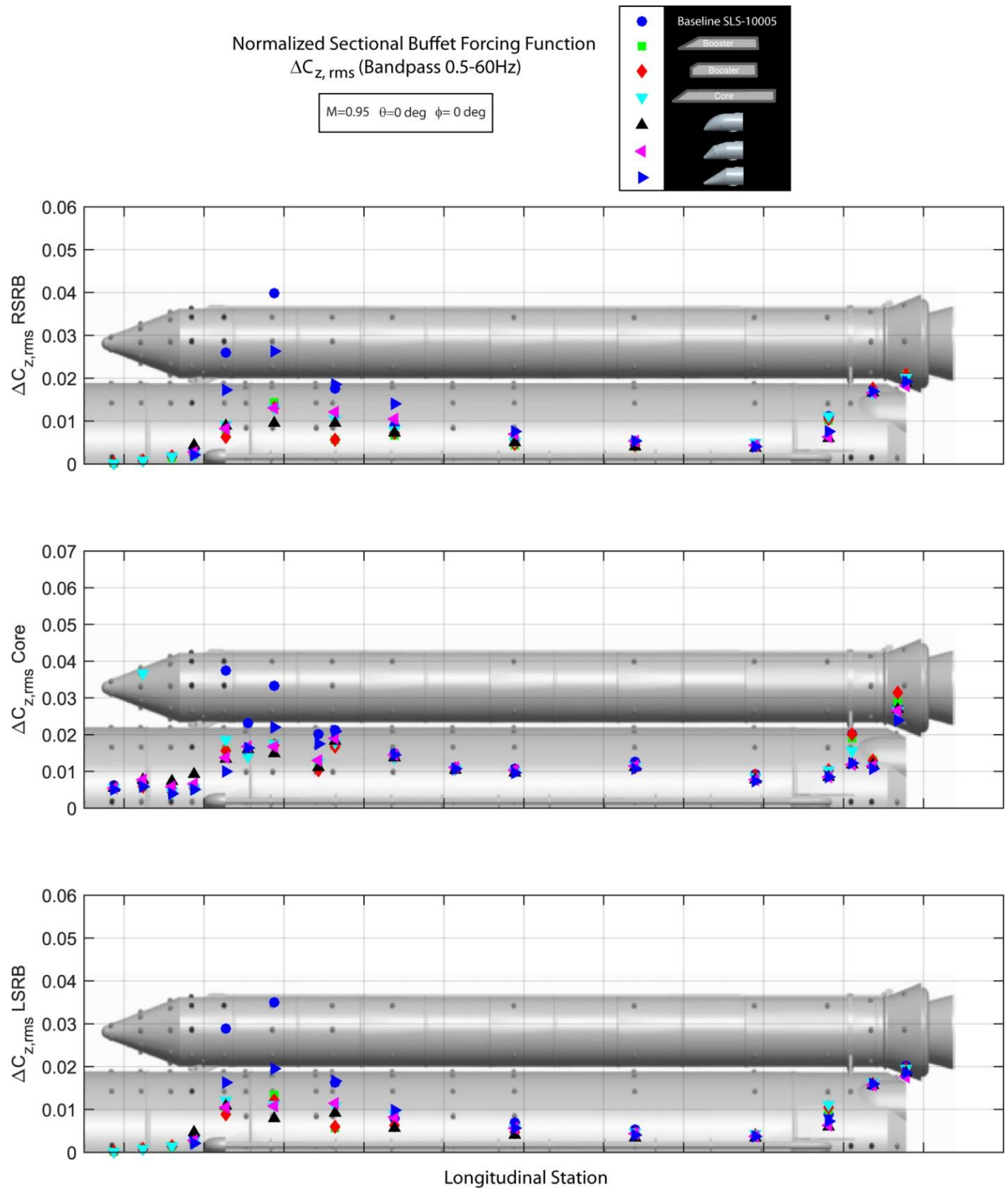

Figure 29. Root-mean-squared trends in z-direction normalized sectional buffet forcing function for baseline and BMO configurations at Mach $0.95, \theta=0^{\circ}$, and $\phi=0^{\circ}$. 
Normalized Sectional Buffet Forcing Function $\Delta \mathrm{C}_{\mathrm{y}, \mathrm{rms}}$ (Bandpass 0.5-60Hz)

$M=0.95 \quad \theta=4$ deg $\phi=0$ deg
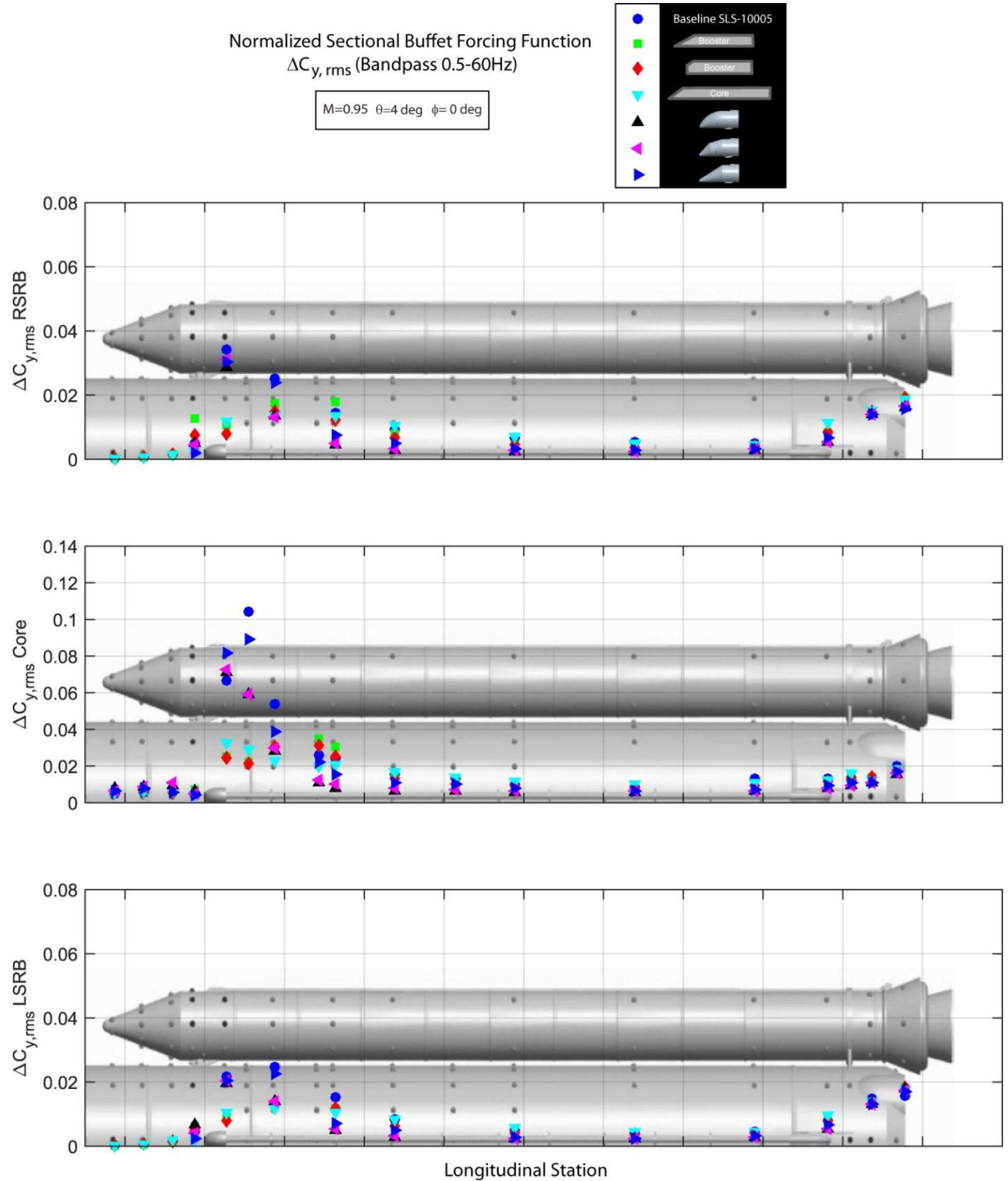

Figure 30. Root-mean-squared trends in y-direction normalized sectional buffet forcing function for baseline and BMO configurations at Mach $0.95, \theta=4$, and $\phi=0^{\circ}$. 

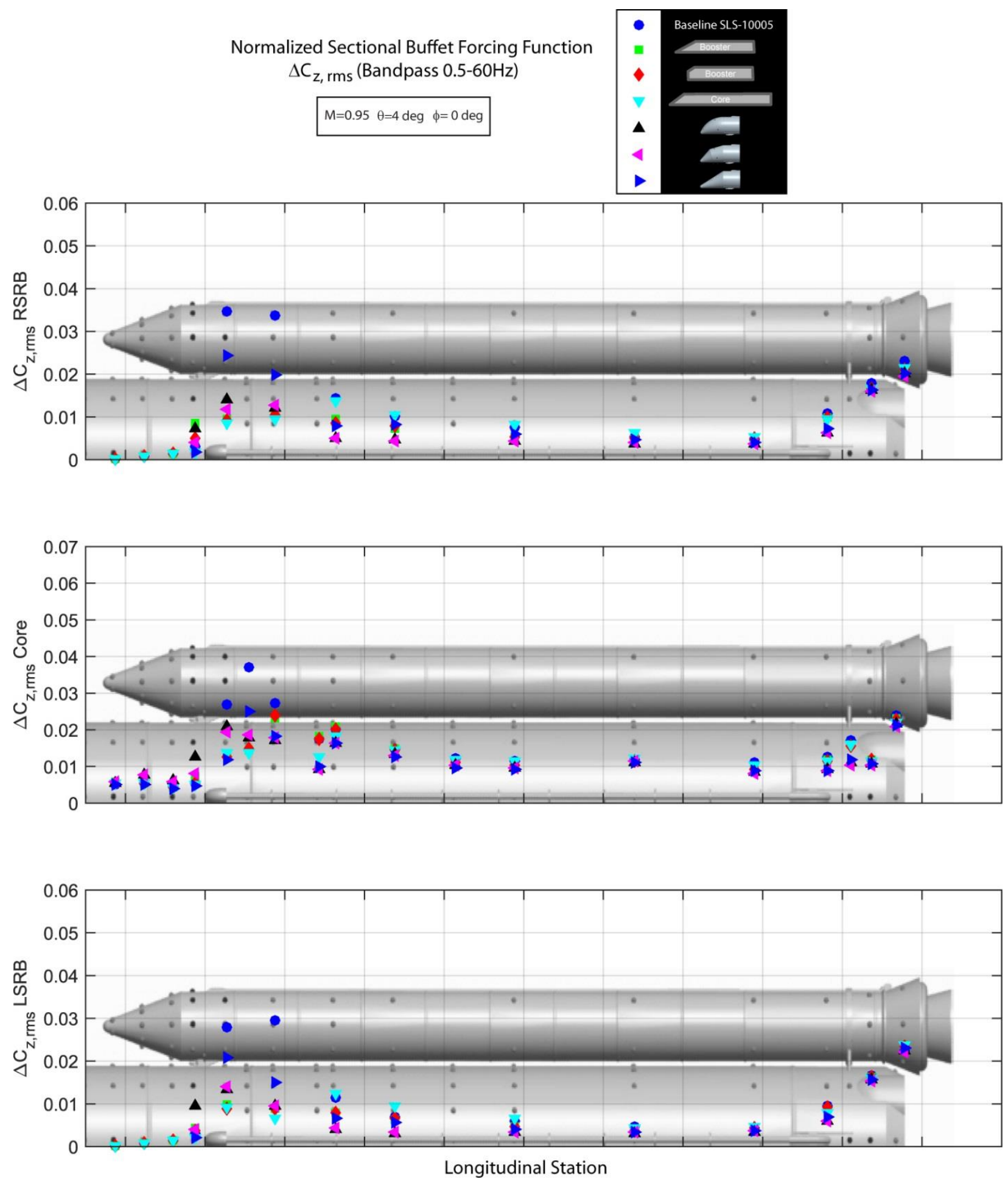

Figure 31. Root-mean-squared trends in z-direction normalized sectional buffet forcing function for baseline and BMO configurations at Mach $0.95, \theta=4$, and $\phi=0^{\circ}$. 

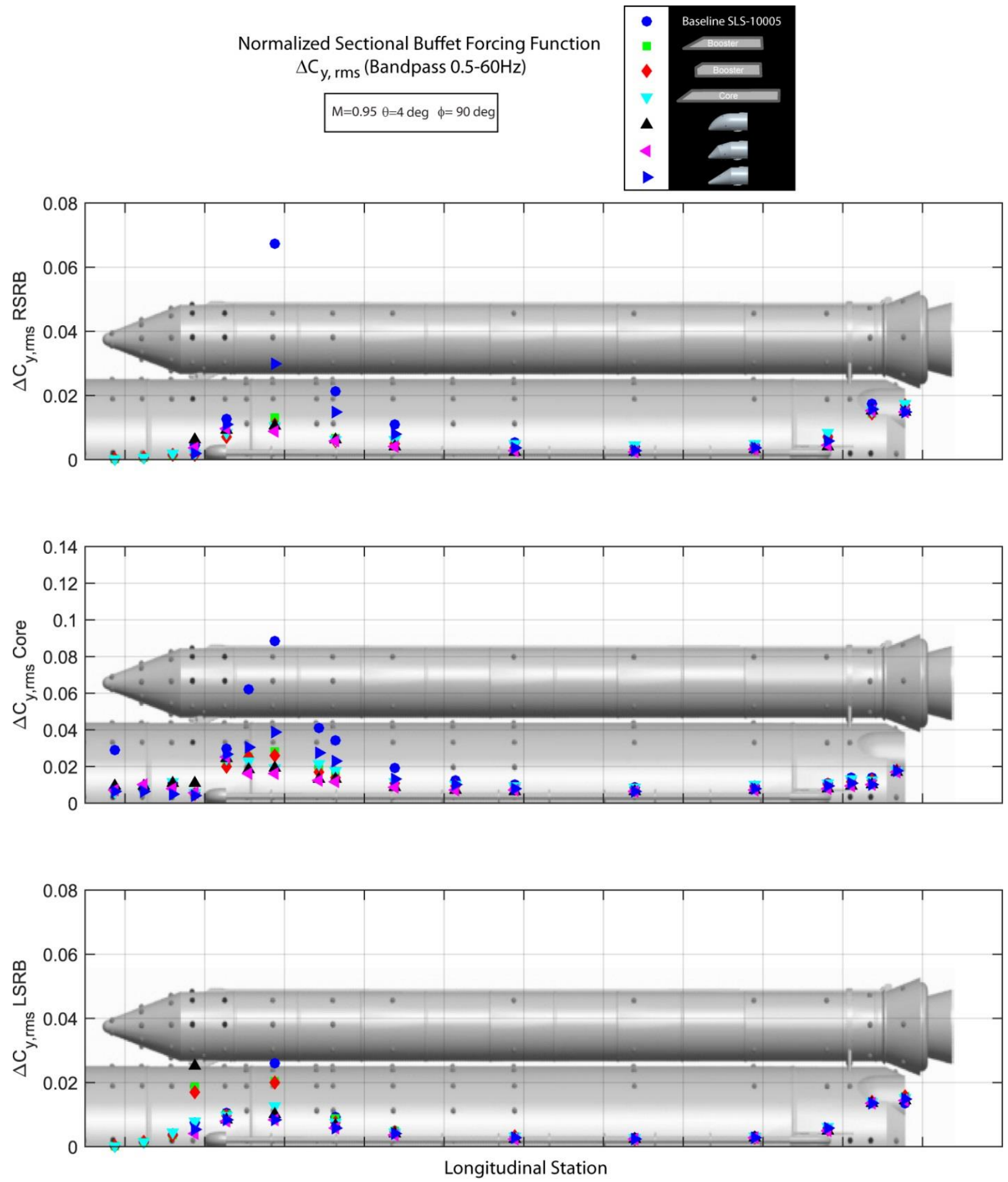

Figure 32. Root-mean-squared trends in y-direction normalized sectional buffet forcing function for baseline and BMO configurations at Mach $0.95, \theta=4$, and $\phi=90^{\circ}$. 

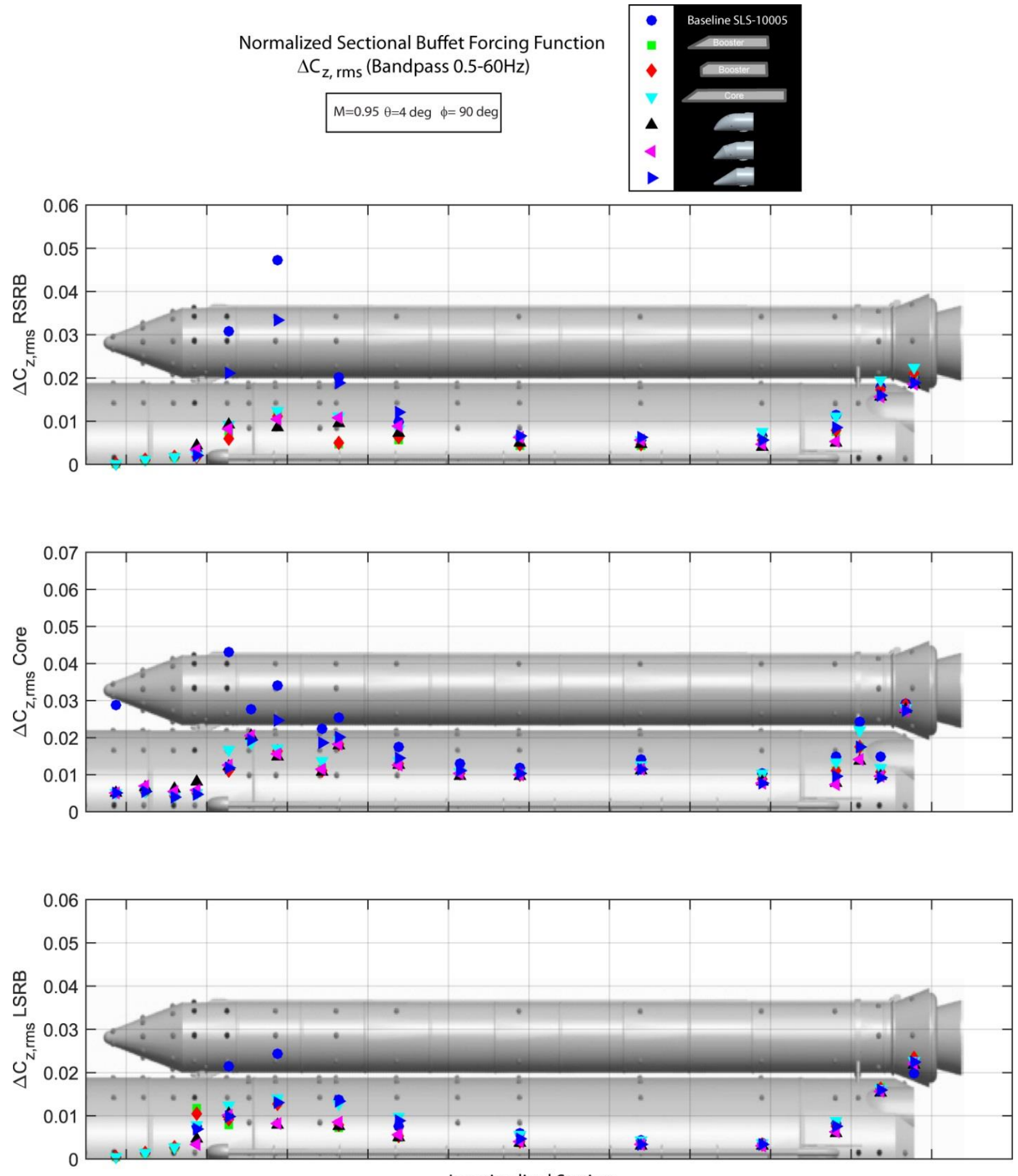

Longitudinal Station

Figure 33. Root-mean-squared trends in z-direction normalized sectional buffet forcing function for baseline and BMO configurations at Mach $0.95, \theta=4$, and $\phi=90^{\circ}$. 

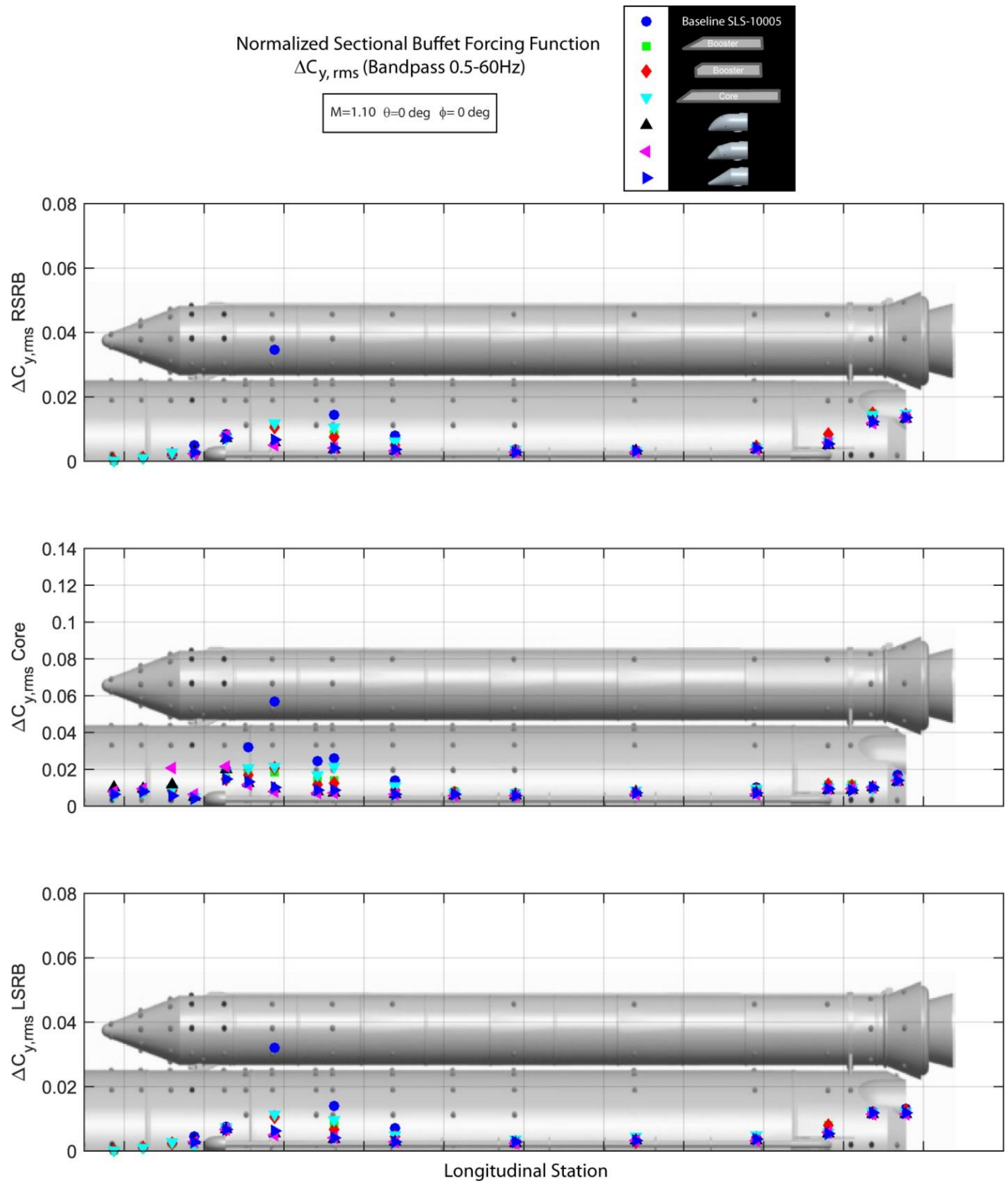

Figure 34. Root-mean-squared trends in y-direction normalized sectional buffet forcing function for baseline and BMO configurations at Mach 1.10, $\theta=0^{\circ}$, and $\phi=0^{\circ}$. 

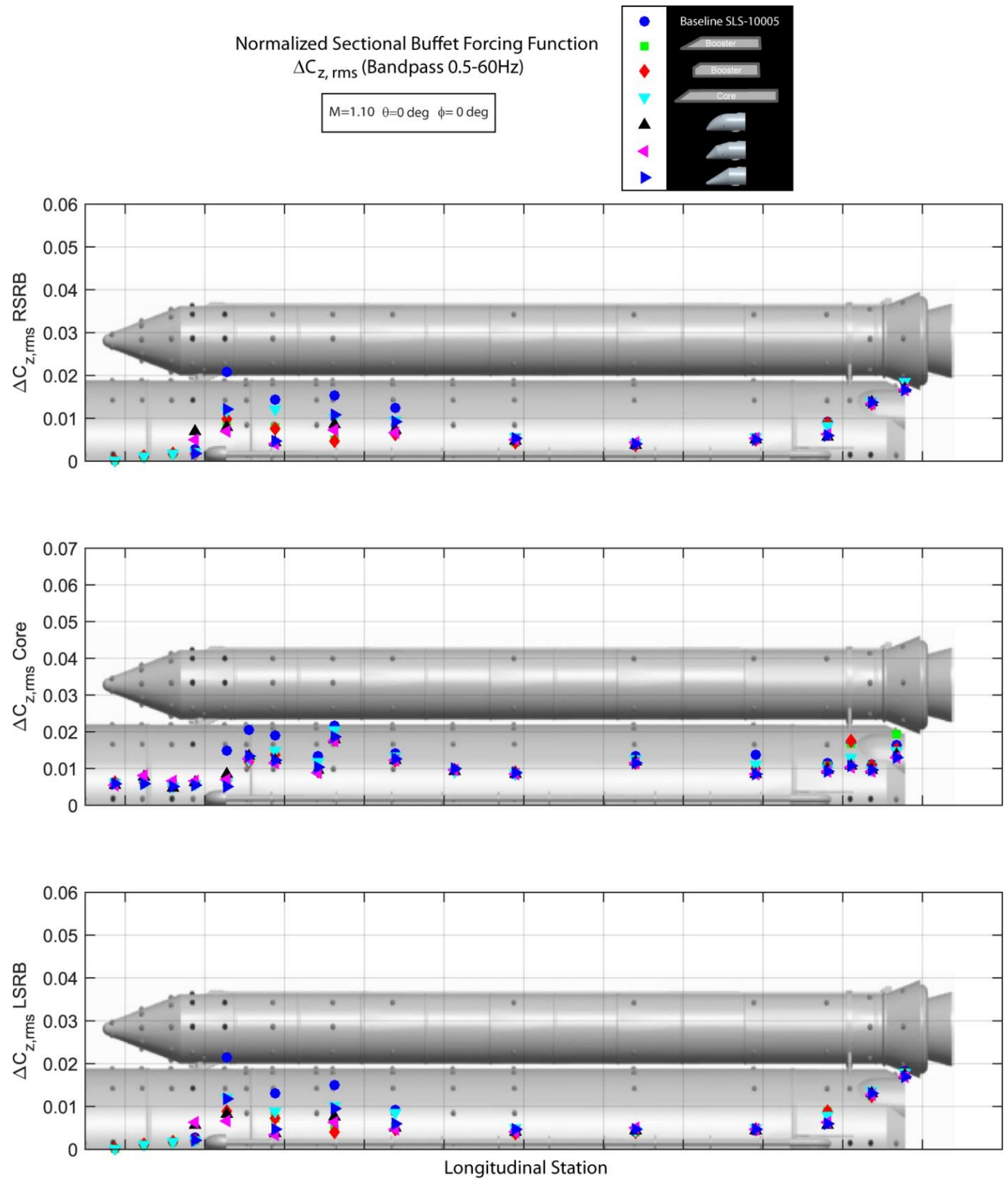

Figure 35. Root-mean-squared trends in z-direction normalized sectional buffet forcing function for baseline and BMO configurations at Mach 1.10, $\theta=0^{\circ}$, and $\phi=0^{\circ}$. 

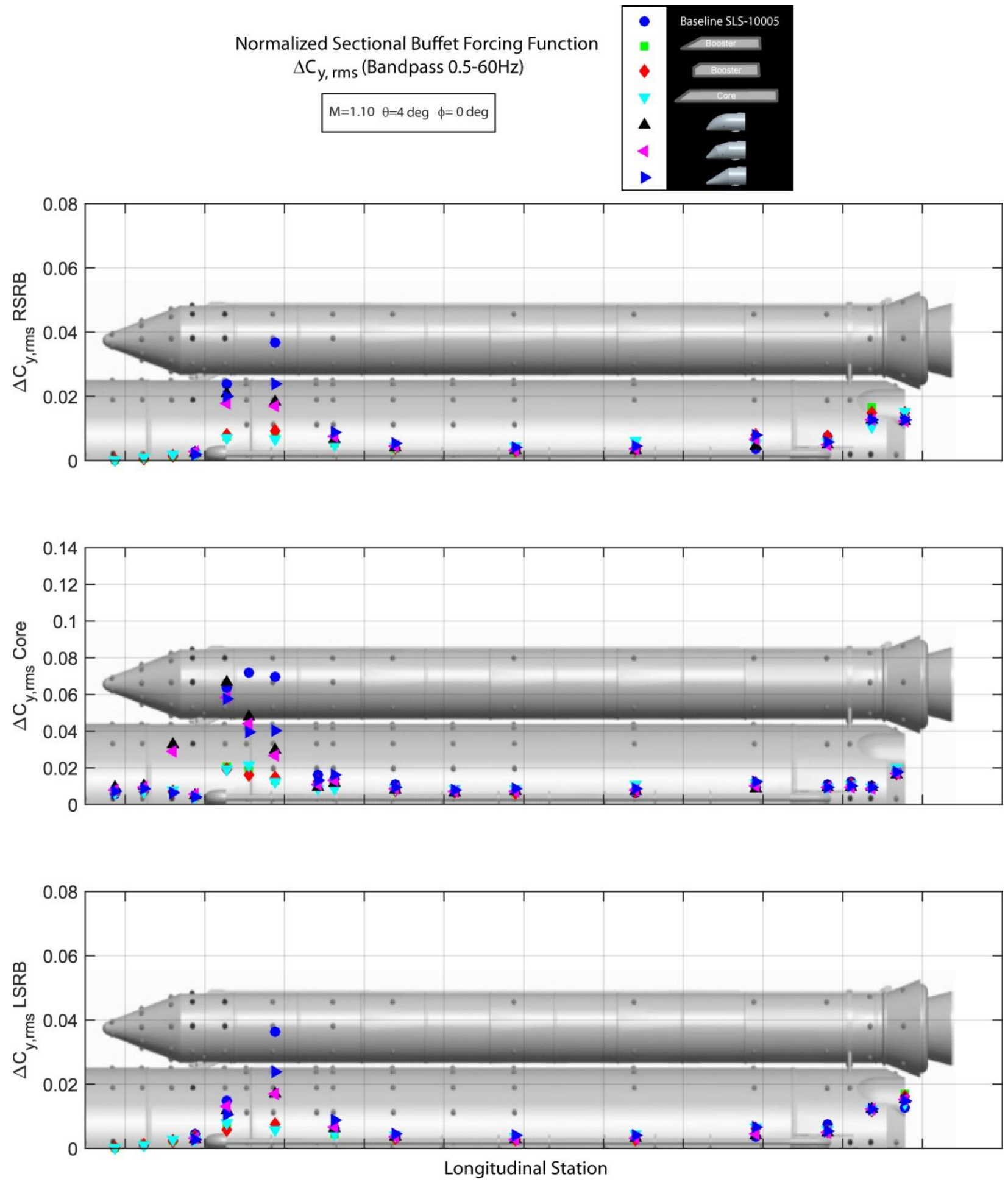

Figure 36. Root-mean-squared trends in y-direction normalized sectional buffet forcing function for baseline and BMO configurations at Mach 1.10, $\theta=4$, and $\phi=0^{\circ}$. 

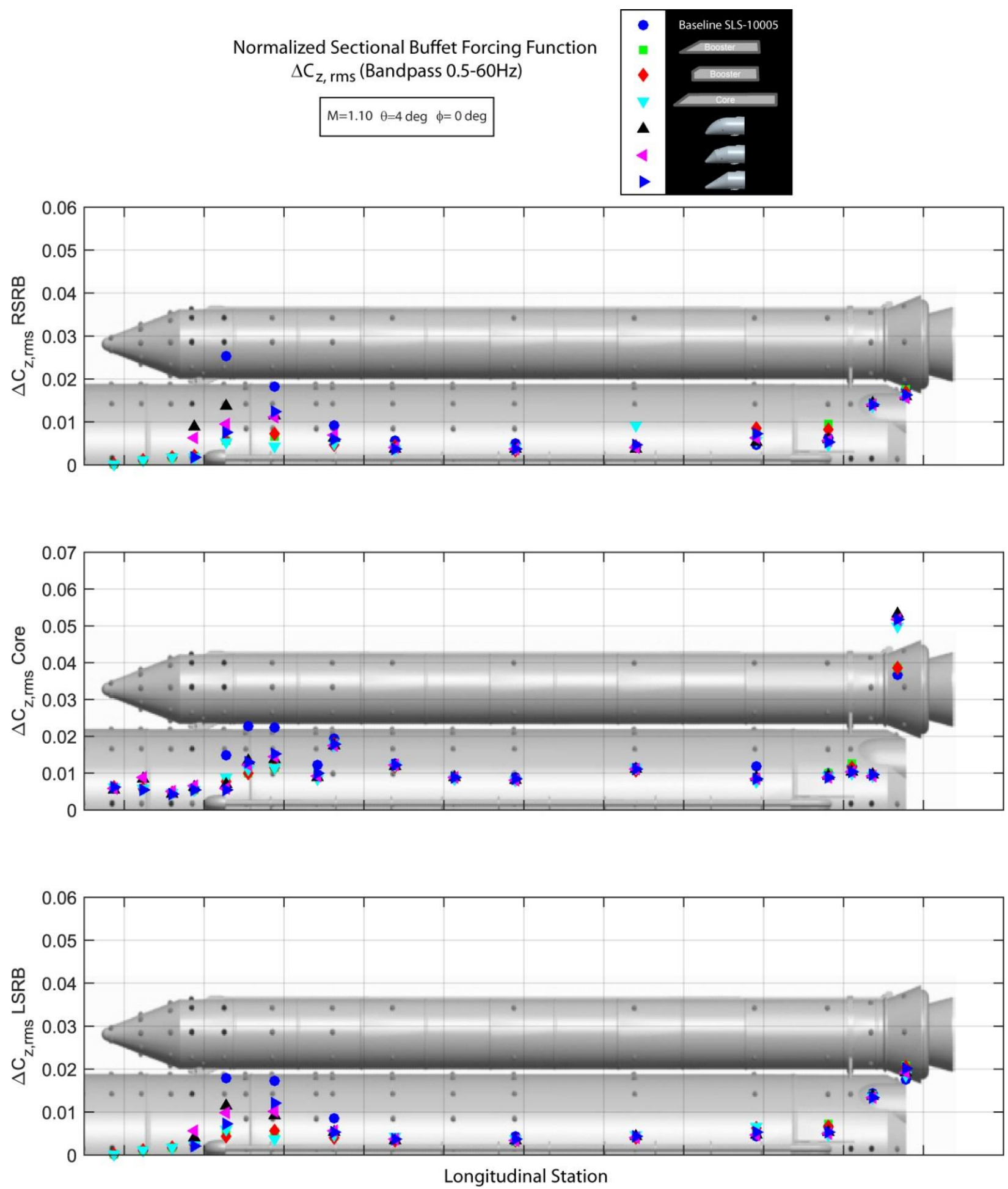

Figure 37. Root-mean-squared trends in z-direction normalized sectional buffet forcing function for baseline and BMO configurations at Mach 1.10, $\theta=4^{\circ}$, and $\phi=0^{\circ}$. 

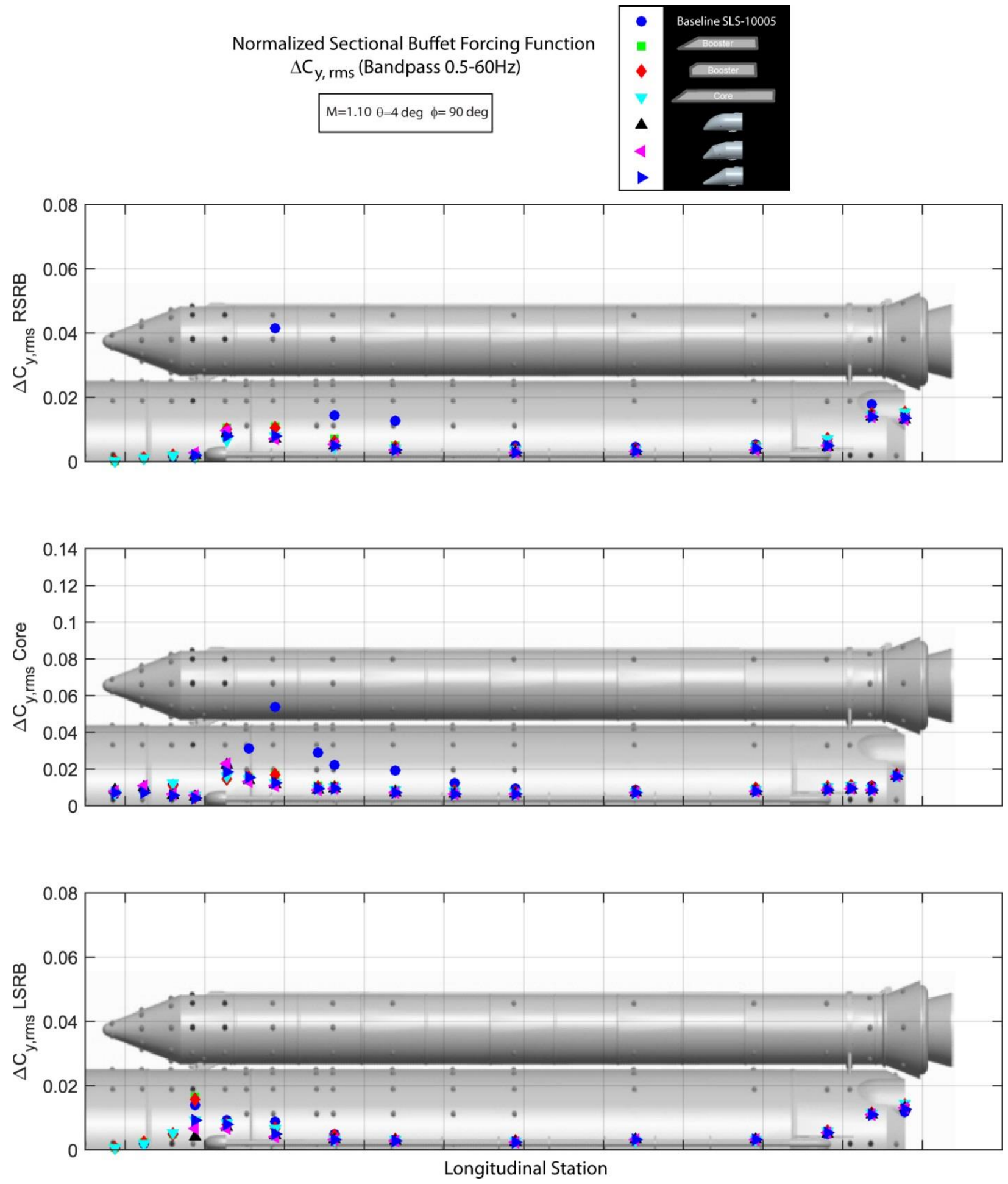

Figure 38. Root-mean-squared trends in y-direction normalized sectional buffet forcing function for baseline and BMO configurations at Mach 1.10, $\theta=4^{\circ}$, and $\phi=90^{\circ}$. 

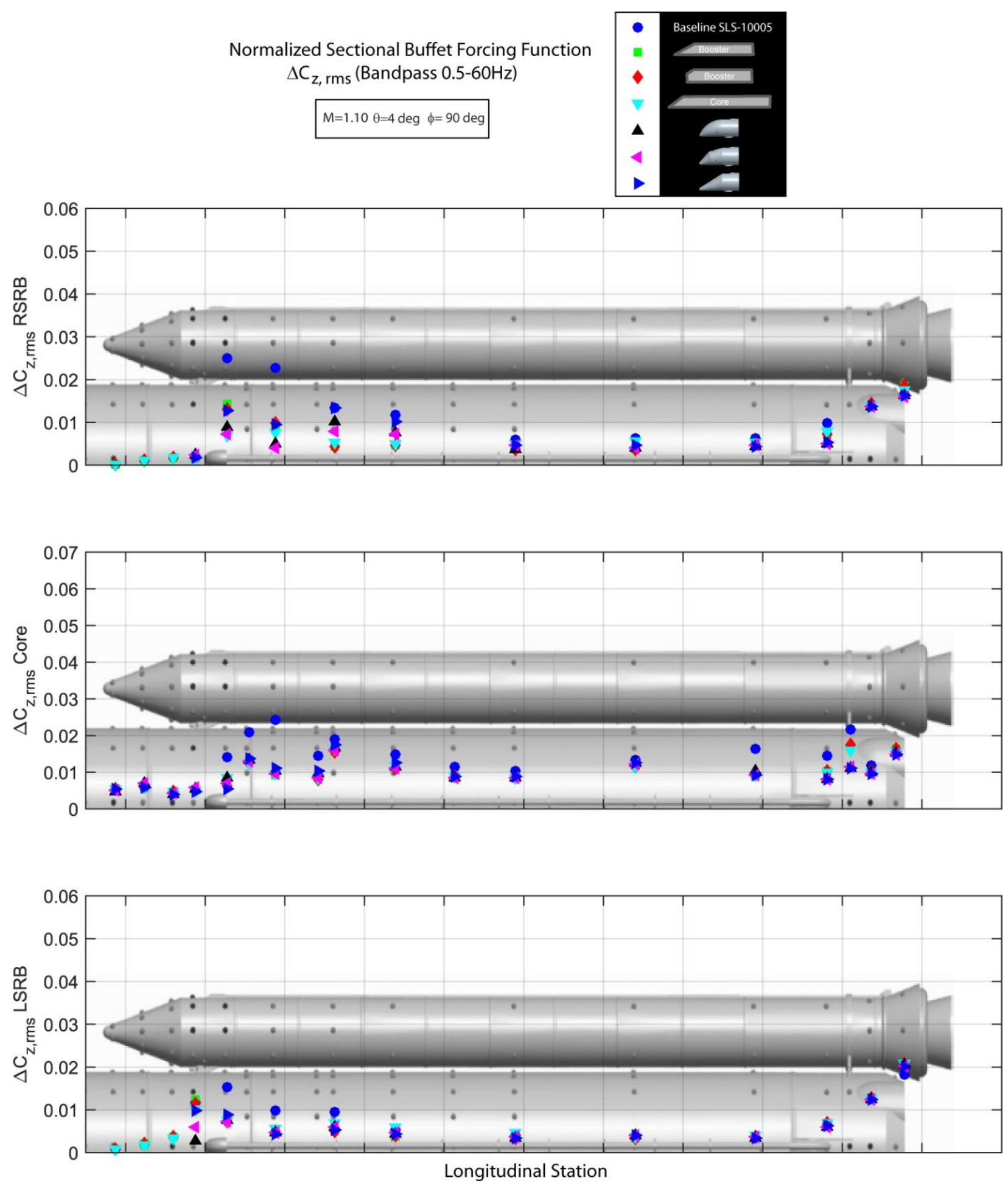

Figure 39. Root-mean-squared trends in z-direction normalized sectional buffet forcing function for baseline and BMO configurations at Mach 1.10, $\theta=4^{\circ}$, and $\phi=90^{\circ}$. 

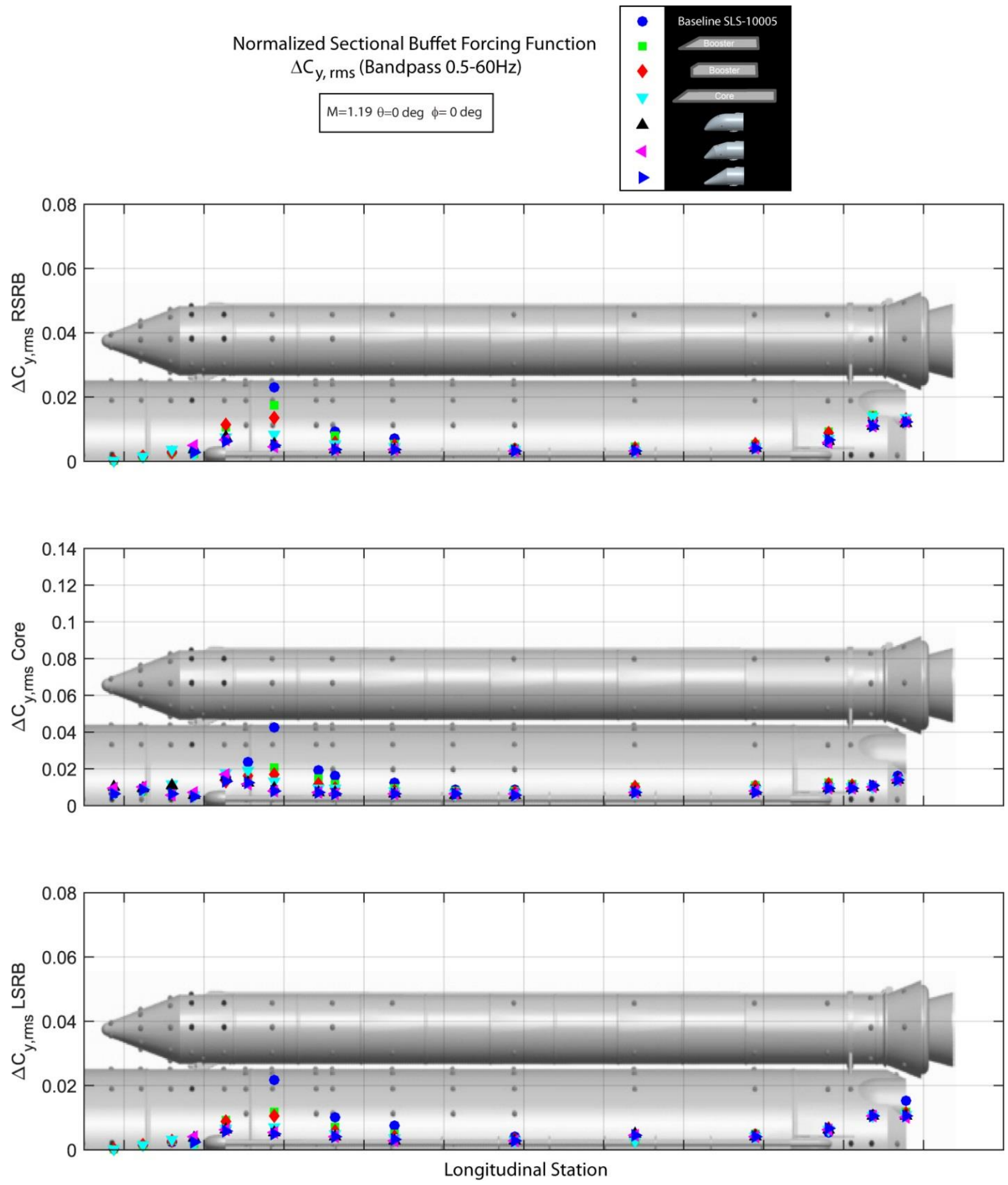

Figure 40. Root-mean-squared trends in y-direction normalized sectional buffet forcing function for baseline and BMO configurations at Mach 1.19, $\theta=0^{\circ}$, and $\phi=0^{\circ}$. 

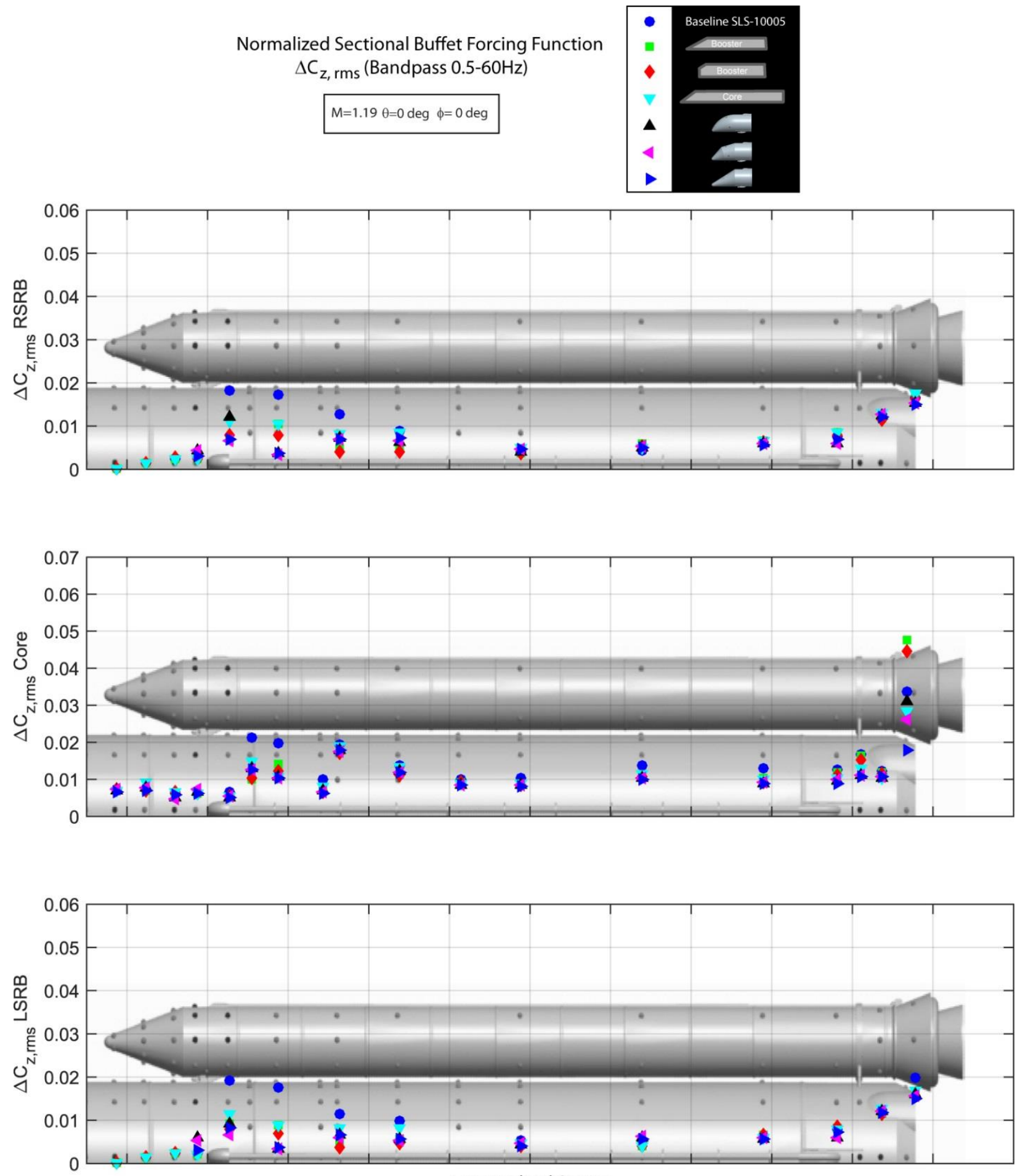

Longitudinal Station

Figure 41. Root-mean-squared trends in z-direction normalized sectional buffet forcing function for baseline and BMO configurations at Mach 1.19, $\theta=0^{\circ}$, and $\phi=0^{\circ}$. 

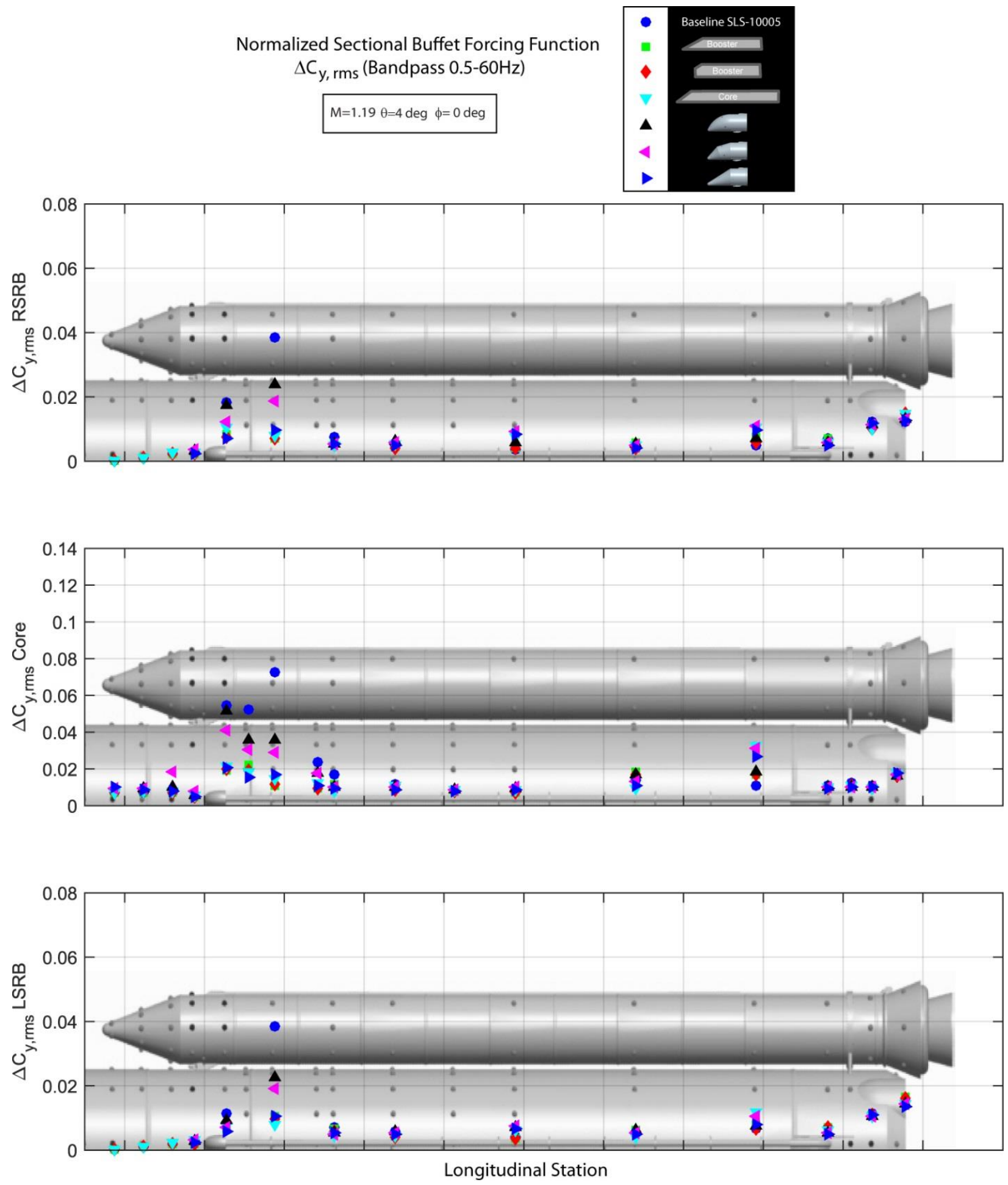

Figure 42. Root-mean-squared trends in y-direction normalized sectional buffet forcing function for baseline and BMO configurations at Mach 1.19, $\theta=4$, and $\phi=0^{\circ}$. 

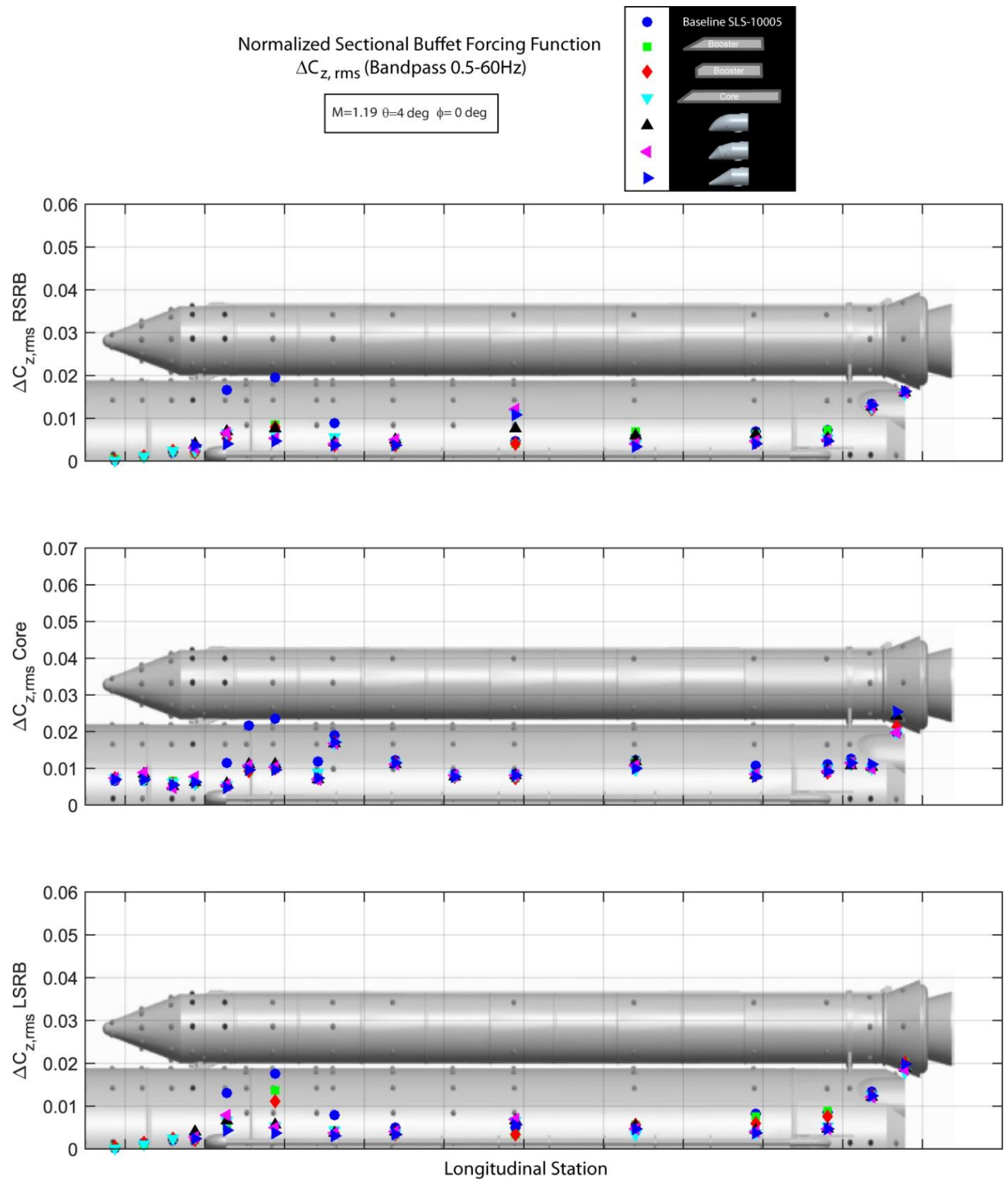

Figure 43. Root-mean-squared trends in z-direction normalized sectional buffet forcing function for baseline and BMO configurations at Mach 1.19, $\theta=4$, and $\phi=0^{\circ}$. 

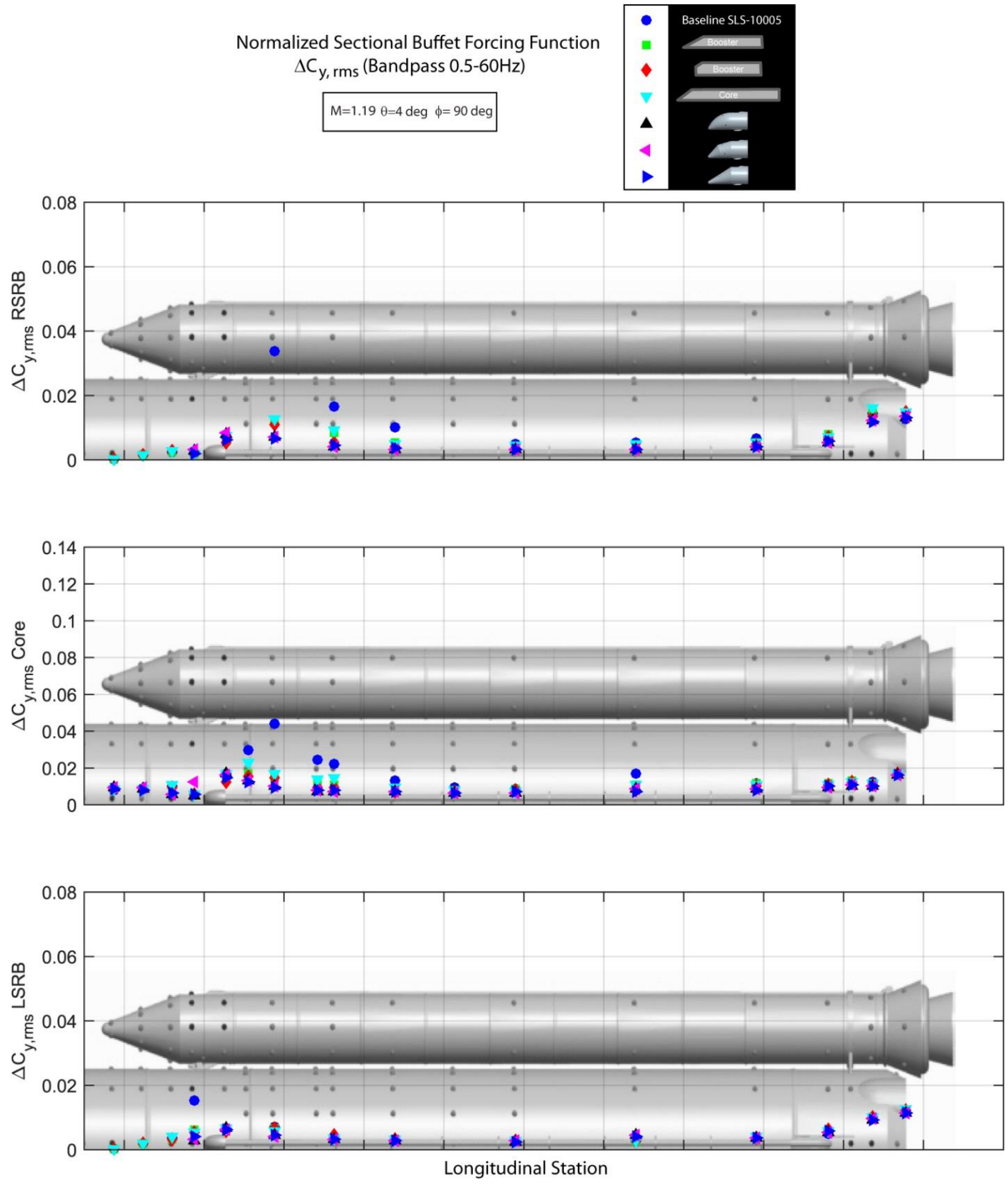

Figure 44. Root-mean-squared trends in y-direction normalized sectional buffet forcing function for baseline and BMO configurations at Mach 1.19, $\theta=4^{\circ}$, and $\phi=90^{\circ}$. 

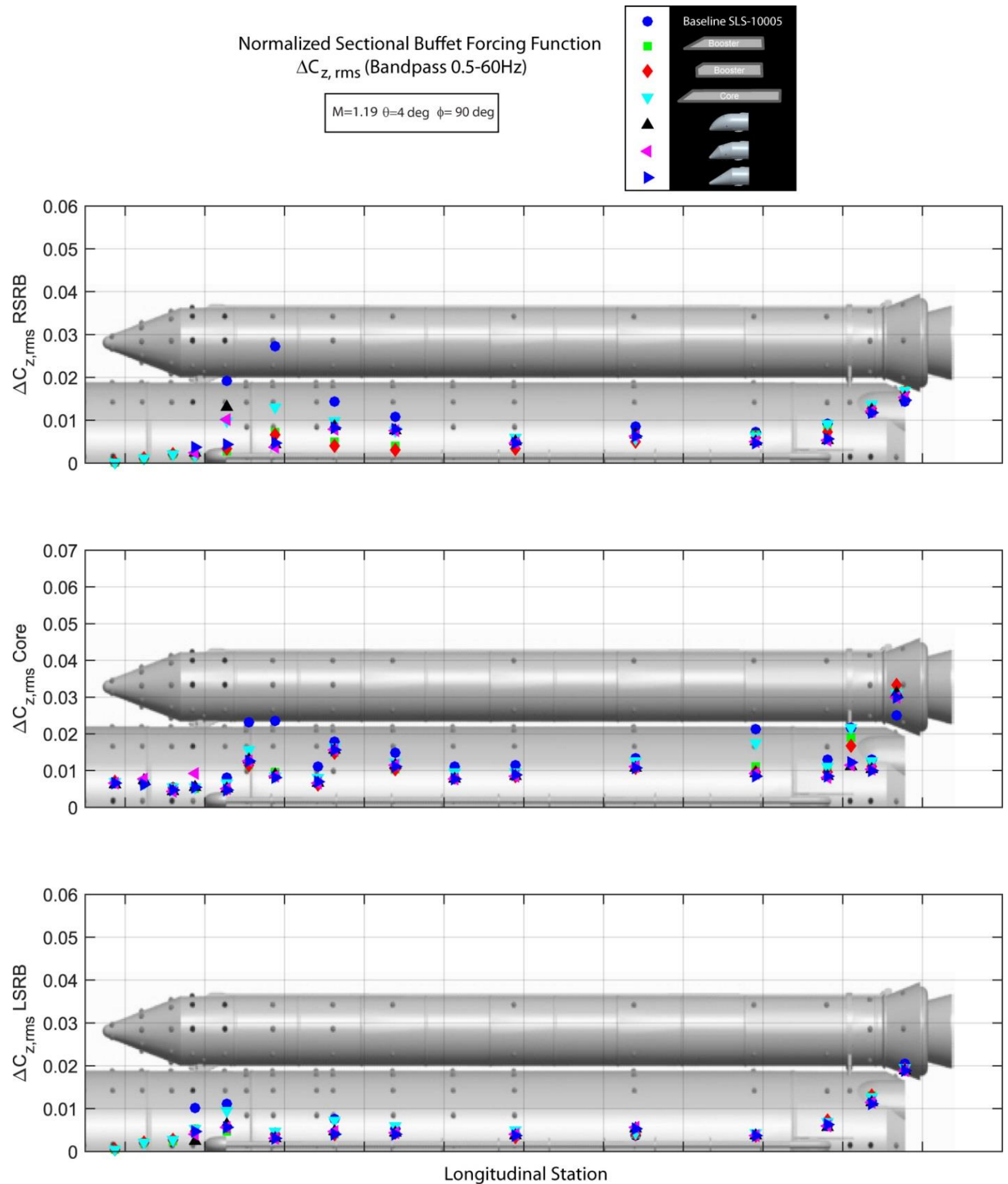

Figure 45. Root-mean-squared trends in z-direction normalized sectional buffet forcing function for baseline and BMO configurations at Mach 1.19, $\theta=4^{\circ}$, and $\phi=90^{\circ}$. 


\section{Percent Reduction in Core BFF RSS Values}

Core Stations 24, 24b, 25, 25b, and 26

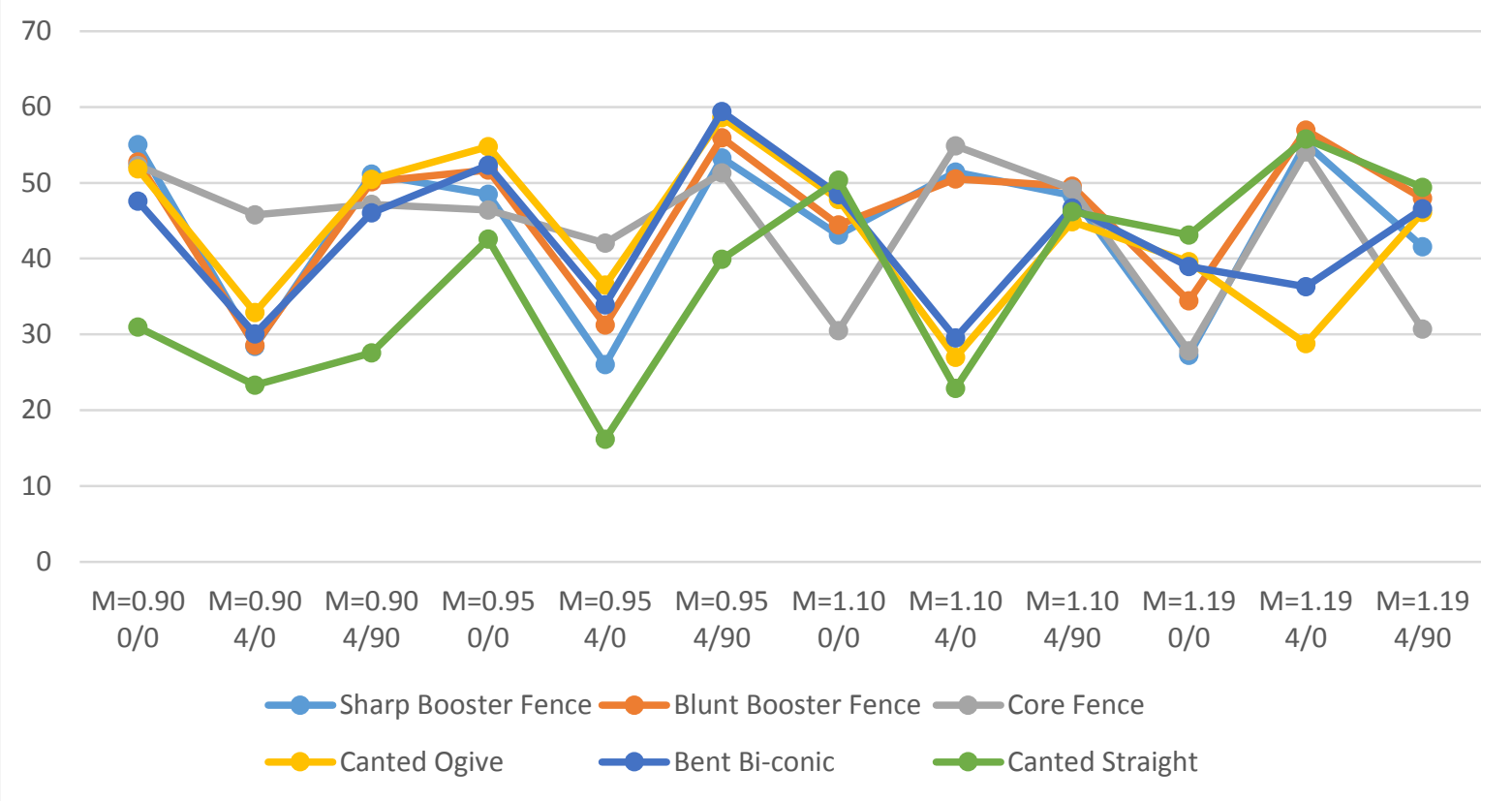

Figure 46. Percent reduction in core BFF root-sum-squared value trends for all BMO configurations for each reference test condition.

\section{Percent Reduction in RSRB BFF RSS Values \\ RSRB Stations 24, 25, and 26}

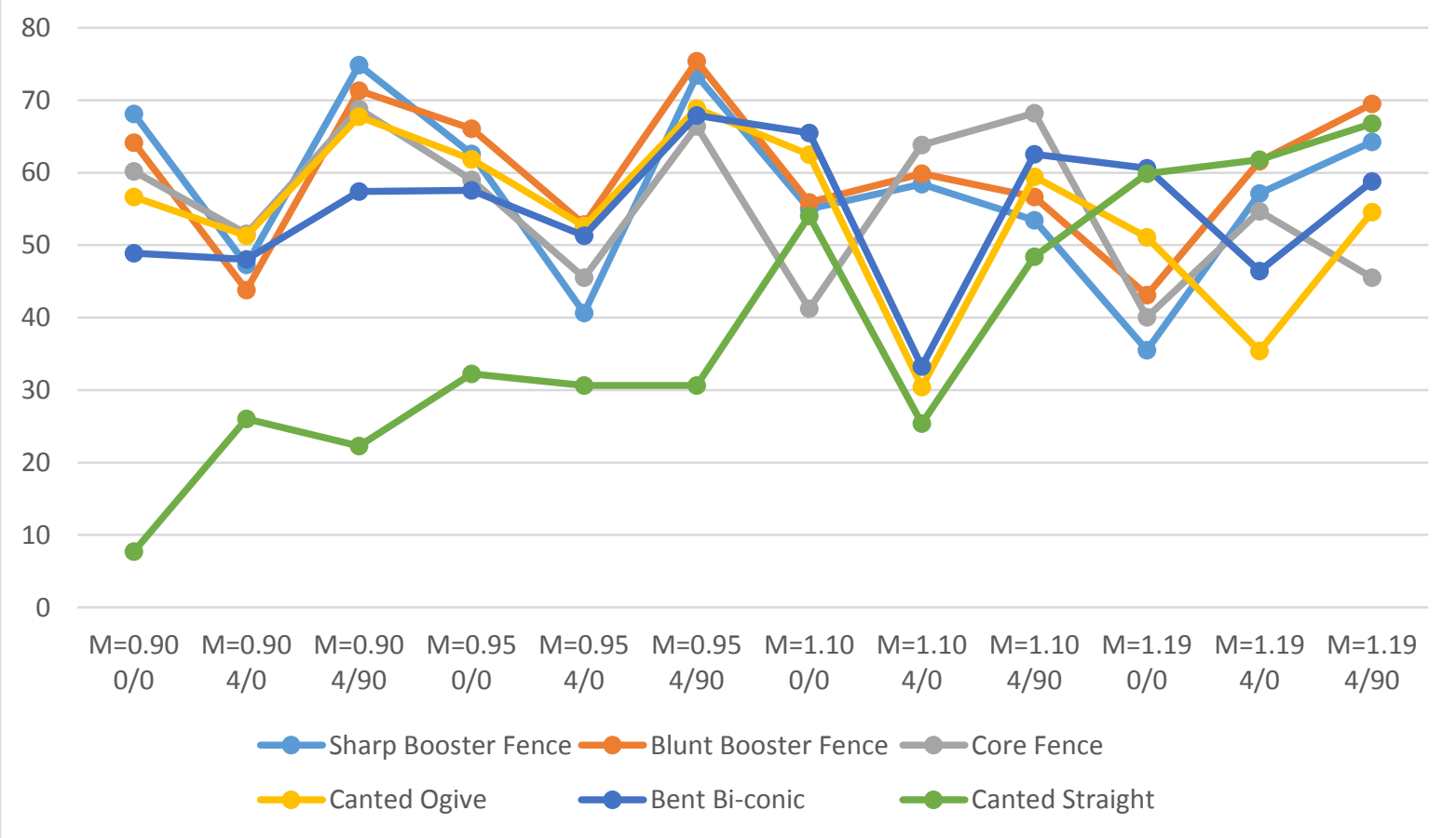

Figure 47. Percent reduction in RSRB BFF root-sum-squared value trends for all BMO configurations for each reference test condition. 


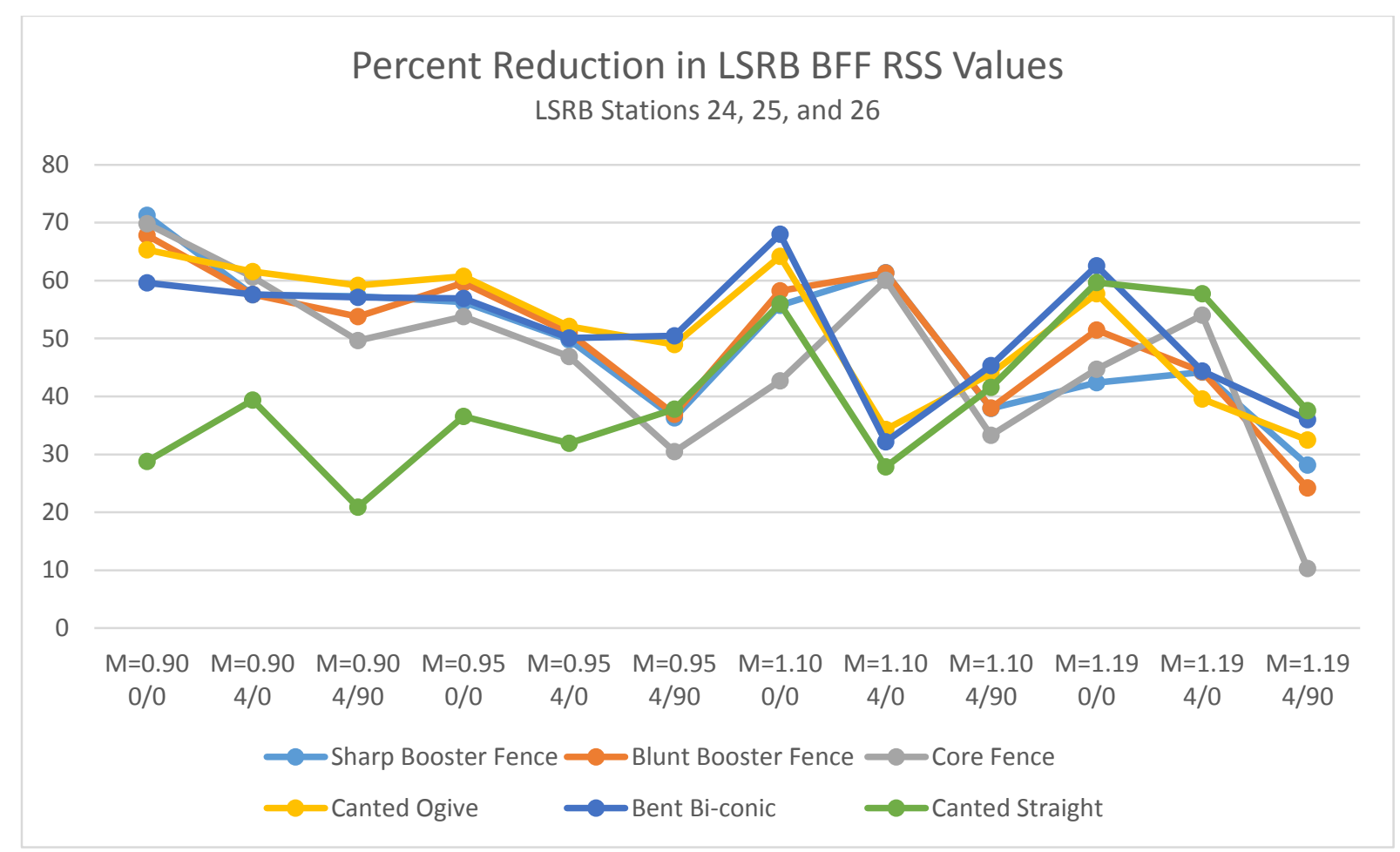

Figure 48. Percent reduction in LSRB BFF root-sum-squared value trends for all BMO configurations for each reference test condition. 